Portland State University

PDXScholar

5-6-1996

\title{
Distribution of lonized and Neutral Halogenated Phenols in an Octanol-water Membrane Model System
}

Robert Word

Portland State University

Follow this and additional works at: https://pdxscholar.library.pdx.edu/open_access_etds

Part of the Physics Commons

Let us know how access to this document benefits you.

Recommended Citation

Word, Robert, "Distribution of lonized and Neutral Halogenated Phenols in an Octanol-water Membrane Model System" (1996). Dissertations and Theses. Paper 5207.

https://doi.org/10.15760/etd.7083

This Thesis is brought to you for free and open access. It has been accepted for inclusion in Dissertations and Theses by an authorized administrator of PDXScholar. Please contact us if we can make this document more accessible: pdxscholar@pdx.edu. 


\section{THESIS APPROVAL}

The abstract and thesis of Robert Word for the Master of Science in Physics were presented May 6, 1996, and accepted by the thesis committee and the department.

COMMITTEE APPROVALS:

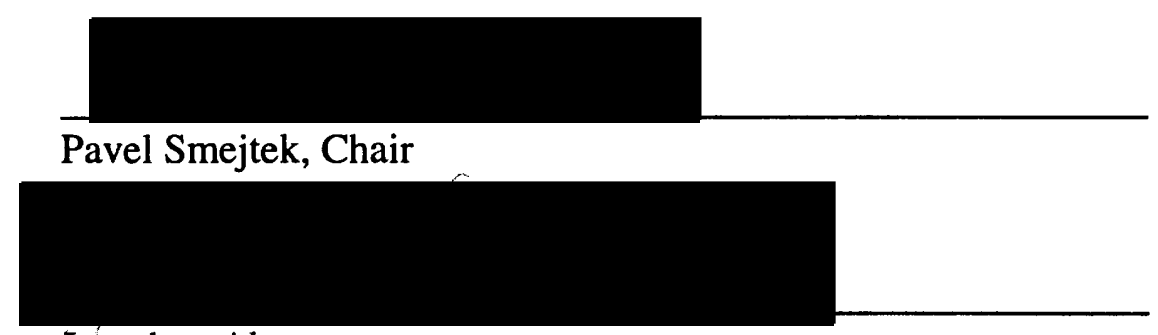

Jonathan Abramson

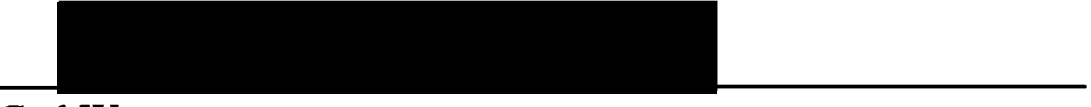

Carl Wamser

Representative of the Office of Graduate Studies

DEPARTMENT APPROVAL:

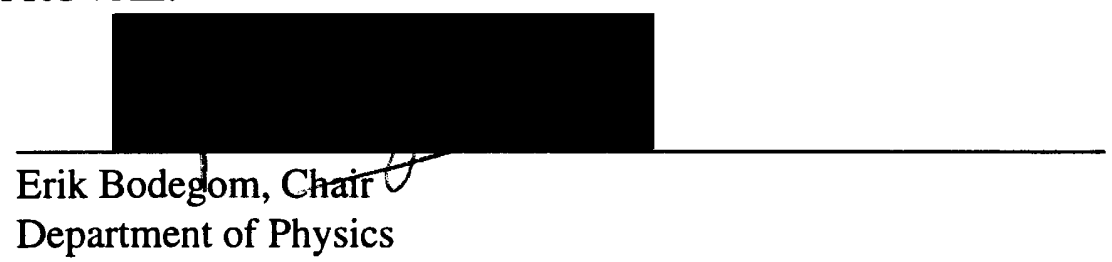

$* * * * * * * * * * * * * * * * * * * * * * * * * * * * * * * * * * * * * * * * * * * * * * * * * * * * * * * * * * * * * * * * * * * * * *$ ACCEPTED FOR PORTLAND STATE UNIVERSITY BY THE LIBRARY

by

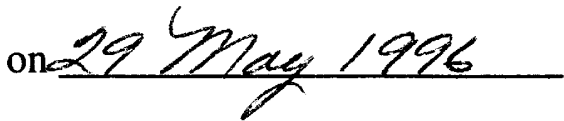




\begin{abstract}
An abstract of the thesis of Robert Word for the Master of Science in Physics presented May 6, 1996.
\end{abstract}

Title: Distribution of Ionized and Neutral Halogenated Phenols in an Octanol-Water Membrane Model System.

The accumulation of xenobiotics, such as halogenated phenols, in soils, sediments, and living organisms is primarily a partitioning process between an aquatic and organic phase. It is traditional to use a bulk octanol-water system to model the partitioning of a compound between complex biological lipid membranes and aqueous media. The octanol-water partition coefficient $K_{O W}$ successfully approximates the lipid membrane-water partition coefficient $K_{\text {LipW }}$ of neutral compounds. However, the ionized species of substituted phenols were shown (Smejtek, et al. 1993; Escher, et al. 1996) to have a higher affinity for lipid membranes than predicted from octanol-water results.

Data on the octanol-water partition coefficients of molecular ions is limited. In order to compare the partitioning of neutral and charged species of halogenated phenols between the lipid membrane-water and octanol-water systems, we measured the $\mathrm{pH}$ dependent distribution of ionized and neutral 2,3,4,6-tetrachlorophenol (TeCP), pentafluorphenol (PFP), pentachlorophenol (PCP), and pentabromophenol (PBP). For the neutral (HA) species of each phenol, $\log K_{O W}$ was found to be 4.28 (TeCP), 2.79 
(PFP), 4.77 (PCP), and approximately 4.67 (PBP). For the ionized $\left(\mathrm{A}^{-}\right)$species of each phenol, $\log K_{\text {Ow }}$ was found to be 0.48 (TeCP), -0.85 (PFP), 1.16 (PCP), and 1.77 (PBP).

These results are compared with sorption data on halophenols in a lipid membrane-water system (Smejtek, et al. 1996). This study shows that ionized halophenols have an affinity for lipid membranes about two to three orders of magnitude greater than for octanol. The usefulness of the octanol-water model as a predictor of lipid membrane-water partitioning for ionizable compounds, such as halogenated phenols, is questioned in view of the present results.

Two thermodynamic $K_{O w}$ prediction models based on molecular properties are discussed in the context of the octanol-water partitioning of ionized and neutral compounds. The partition coefficients predicted by the molar volume based model (Gobas, et al. 1988) correlates with the experimental $K_{O W}$ results of this study better than $K_{\text {OW }}$ results predicted from the molecular surface area based model (Smejtek, et al. 1996).

Results of this study support the hypothesis that partitioning of xenobiotics in the octanol-water system is fundamentally different than partitioning in lipid membrane-water systems. 


\title{
DISTRIBUTION OF IONIZED AND NEUTRAL HALOGENATED PHENOLS IN AN OCTANOL-WATER MEMBRANE MODEL SYSTEM
}

\author{
By \\ Robert Word
}

A thesis submitted in partial fulfillment of the requirements for the degree of

\author{
MASTER OF SCIENCE \\ in \\ PHYSICS
}

Portland State University

1996 


\section{Acknowledgments}

I am very grateful to Dr. Pavel Smejtek and Shanru Wang for their support and guidance over the last year. I also wish to thank Margie Fyfield, Piet O. Schmidt, Isolde Sieder, and Sven Hensler for sharing all the struggles and the spoils. And I am especially grateful to Michelle for enduring all the evenings and weekends that I spend away from home in the lab. 
Distribution of Ionized and Neutral Halogenated Phenols in an Octanol-Water Membrane Model System

Table of Contents

I. Introduction 1

II. Theory

Ideal Partitioning ...................................................................................... 9

Solutions of Two Species and the Distribution Function ............................ 11

The Octanol and Aqueous Phases ............................................................. 13

UV-Visible Spectrophotometry ............................................................... 15

Beer's Law and Absorption Spectroscopy ................................................... 18

DU-7 Spectrophotometer ........................................................................... 20

III. Materials and Methods

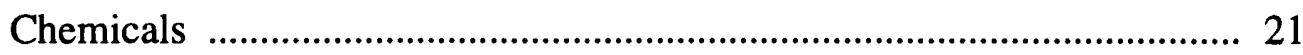

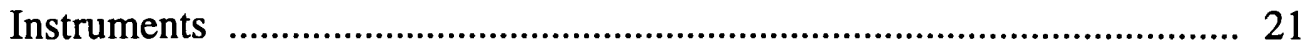

Preparation of the Octanol and Buffer Phases .......................................... 22

Extinction Coefficients ......................................................................... 23

Octanol-Water Partitioning ……………………….............................. 25

IV. Results

Extinction Coefficients .............................................................................. 27

Octanol-Water Partitioning .......................................................................... 46

V. Discussion

Molar Volume Model of Two-Phase Partitioning …………………........ 58

Molecular Surface Model of Two-Phase Partitioning .................................. 65

Distribution of Halophenols in a Lipid membrane-water System .............. 73

Limits of the "Shake-Flask" Method ........................................................... 76

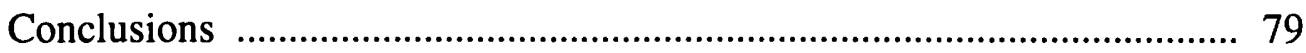

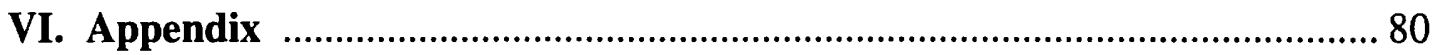

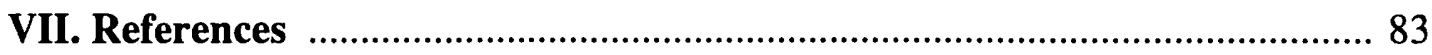




\section{List of Tables}

\section{Section IV. Results}

1. Extinction coefficient data for 2,3,4,6-TeCP ……......................................... 30

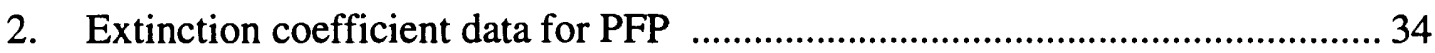

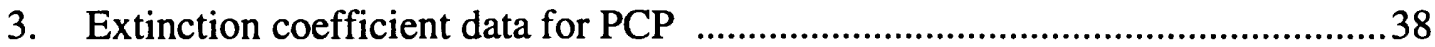

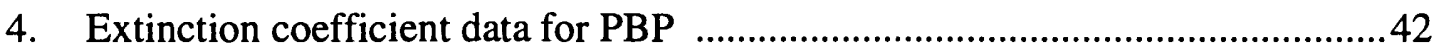

5. Distribution parameters of halogenated phenols ............................................. 48

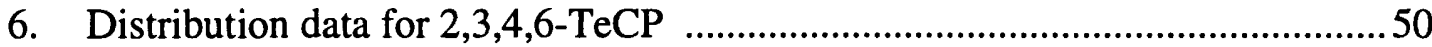

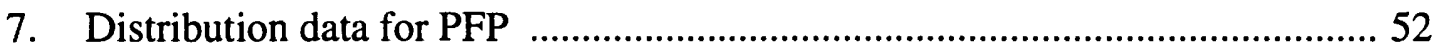

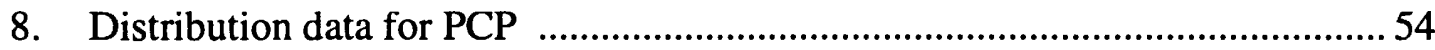

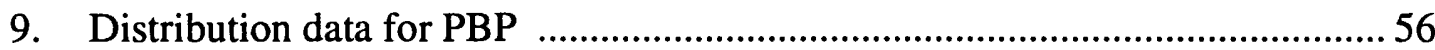

\section{Section V. Discussion}

10. Molar volumes of halophenols, halophenolates,

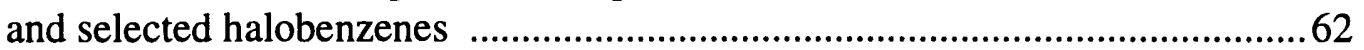

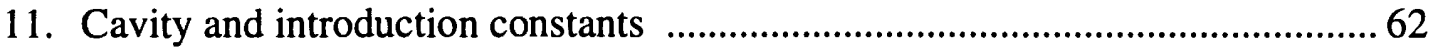

12. Molecular surface areas of halophenols, halophenolates, and selected halobenzenes ............................................................................... 70

13. Slope and intercepts results for linear regression curve fitting ....................... 70

14. Octanol-water and lipid membrane-water partitioning parameters for halophenols and halophenolates

\section{Section VI. Appendix}

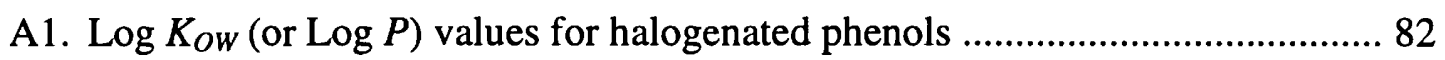




\section{List of Figures}

\section{Section I. Introduction}

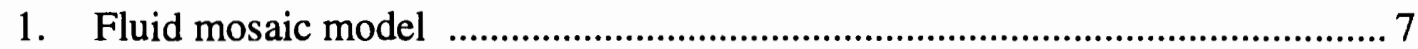

2. Space filling models of PCP, 1-octanol, and phosphatidylcholine .................. 8

3. Molecular structure of halogenated phenols ................................................. 8

\section{Section II. Theory}

4. Energy level diagram of singlet electronic states ............................................ 17

5. Left, Vibrational states superimposed on electronic states Right, Spectral broadening due to vibrational transitions .............................. 17

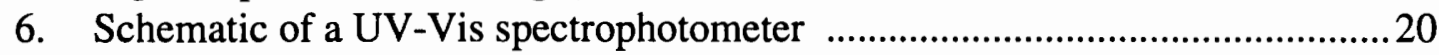

\section{Section IV. Results}

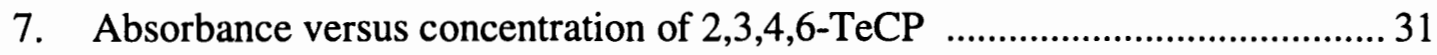

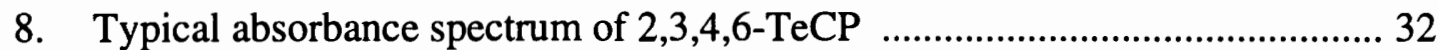

9. Absorbance spectrum of neutral 2,3,4,6-TeCP ............................................... 33

10. Absorbance versus concentration of PFP .................................................. 35

11. Typical absorbance spectrum of PFP ……….............................................. 36

12. Absorbance spectrum of neutral PFP ......................................................... 37

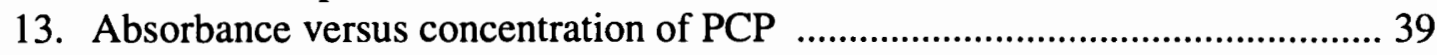

14. Typical absorbance spectrum of PCP ……................................................... 40

15. Absorbance spectrum of neutral PCP ............................................................ 41

16. Absorbance versus concentration of PBP ..................................................... 43

17. Typical absorbance spectrum of PBP ........................................................ 44

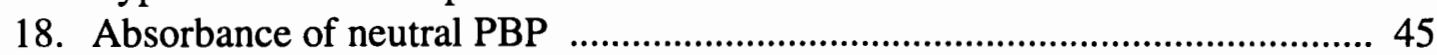

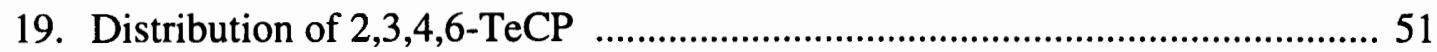

20. Distribution of PFP ............................................................................... 53

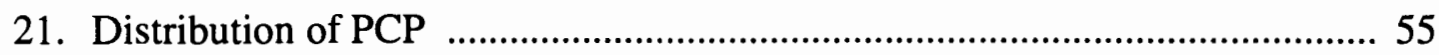

22. Distribution of PBP ……....................................................................... 57

\section{Section V. Discussion}

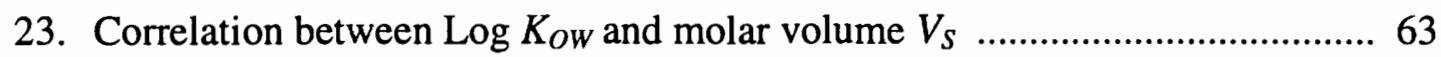

24. $\log K_{O W}$ versus $V_{S}$ for ionized and unionized halogenated phenols ............... 64

25. Correlation between $\log K_{O W}$ and molecular surface area ............................ 71

26. Log $K_{O W}$ versus $M S A$ for ionized and unionized halogenated phenols ........... 72

27. Comparison between the lipid membrane-water and octanol-water distribution of 2,3,4,6-TeCP and PCP ........................................................ 75

28. Apparent limit of the "shake flask" method ................................................ 79 


\section{Introduction}

The ecological introduction pathways and fate of xenobiotics, such as pentachlorophenol (PCP) and its derivatives, is of prime importance to environmental impact studies.

Since the 1930's and until the 1980's (when PCP was banned for general use by most countries) chlorinated phenols were widely used in the wood products industry and agriculture. As an effective biocide, PCP was used extensively as an insecticide, a fungicide, a herbicide, and a wood preservative.

Green lumber was often treated with PCP to prevent mold and mildew staining. As a result, many lumber yards are extensively polluted with PCP ladened soil. Recently, there was public concern over water pollution introduced from polluted lumber yards inundated during winter flooding in the Willamette Valley.

Polychlorinated phenols are often degradation byproducts of PCP. The major degradation products of PCP are 2,4,6-trichlorophenol (an established carcinogen) and 2,3,4,6-tetrachlorophenol. Other contaminates, including polychlorinated dibenzo-pdioxins introduced from the production of PCP, are also highly toxic. In the wood pulp bleaching process employing chlorine, lignin macromolecules are broken down into products including chlorophenols (Mussalo-Rauhamaa, et al. 1989). Chlorinated phenols are also produced by the degradation of PCP by sunlight ${ }^{1}$ and from waste produced by municipal incinerators.

1 The half-life of PCP under daily exposure to sunlight is about 3.5 days (Hattemer-Frey \& Travis 1989). 
Chlorophenols absorb through intact skin, lung tissue, or directly into open wounds, but $99.9 \%$ of the $11 \mu \mathrm{g}$ PCP introduced, per day, ${ }^{2}$ to a non-occupationally exposed US resident is by the ingestion of contaminated foods (Hattemer-Frey \& Travis 1989). In the UK, the estimated daily intake of PCP by non-occupational exposed persons was $4.53 \mu \mathrm{g}$ (Wild \& Jones 1992).

The major sinks of PCP in the body, and presumably other chlorinated phenols, are the liver, the kidneys, and the brain. In the US, the total internal burden of PCP per person is approximately $673 \mu \mathrm{g}$. In the UK, the PCP body burden was found to be 550 $\mu \mathrm{g}$. Pentachlorophenol is metabolized by the kidneys and excreted in urine. After approximately 84 days, one-half of a dose of PCP is metabolized and expelled (Wild \& Jones, 1992).

PCP is lethal in humans when the median body concentration reaches $40-100$ $\mu \mathrm{g}$ per gram of body tissue (Mussalo-Rauhamaa, et al. 1989). For a $70 \mathrm{~kg}$ adult, this concentration corresponds to a total body burden of 3 to $7 \mathrm{~g}$ (about $10^{4}$ times the average burden). Symptoms of PCP poisoning include loss of appetite, respiration difficulties, headaches, and clinical evidence of kidney and liver dysfunction and skin and eye disease.

To evaluate the fate and toxicity of xenobiotics, such as chlorinated phenols, for environmental studies and for drug synthesis research we turn to models of the complex soil, sediment, and biological systems.

2 Based on the PCP concentration in urine samples. 


\section{The Octanol-Water Partition Coefficient, $K_{\text {OW }}{ }^{\cdot}$}

The accumulation of hydrophobic toxins in soils, solid wastes, and biological tissue develops through the processes of adsorption and sorption (Wang, et al. 1993). A solute in aqueous solution must first be adsorbed to the surface of the solid and then may be sorbed into the body of the solid. The sorption of toxins by soils, sediments, organic wastes, and biological tissue is primarily a function of partitioning between water and an organic phase. This is a reasonable assumption, since organic matter, such as dead and living microorganisms, comprise the bulk of soils and waste.

The membranes of cells, nuclei, mitochondria, and other cellular structures are all constructed of lipid membranes. The combination of a hydrophilic polar headgroup and hydrophobic hydrocarbon tails allow lipids to form micelles, and more importantly, bilayer vesicles in aqueous media. In addition, a large percentage of biological membranes consist of surface and integral proteins. These proteins perform energy conversion, transport, identification, locomotion, and regulatory functions. The arrangement of lipids and proteins that form cell membranes is defined in the fluid mosaic model (Figure 1) developed by Singer and Nicolson in 1972 (Zubay 1983). As with most real cases, researchers have tried to find simple model systems to represent the biological membrane-water system.

1 -octanol is the traditional substitute for lipids in membrane-water partitioning models. Although octanol is smaller and lighter, it has a similar structure to biological lipids, including a polar $\mathrm{OH}$ group and a hydrophobic hydrocarbon tail (Figure 2). The 
partitioning property of a solute between octanol and water is called the octanol-water partition coefficient $K_{\text {ow }}$ or $P_{O W}$, and is usually reported as a decadic logarithm. $K_{O W}$ is obtained by mixing a known concentration of solute in octanol with water (or water with octanol) and after separating the phases, by measuring the final solute concentration of each phase. The partition coefficient is then given by

$$
K_{\text {OW }}=\frac{C_{\text {octanol }}}{C_{\text {water }}},
$$

where $C$ is the concentration of each phase.

Many methods of measuring $\mathrm{K}_{\mathrm{OW}}$ have been developed including Revere Phase High Performance Liquid Chromatography (RP-HPLC), a generator column method, and a "slow-stirring" method. In this work, we used the traditional "shake-flask" method to mix the phases and UV-Vis spectrophotometry to measure concentrations. Partition coefficients have been successfully measured for thousands of compounds and many compiled references are available from the literature (Hansch, et al. 1995). Octanol water partition coefficients are widely used in drug research and environmental impact studies.

Since measurements of partition coefficients are time-consuming and sometimes very difficult, various computer programs and estimation techniques have been developed over the last twenty years. Many authors have correlated $K_{O W}$, with varied success, to other physical properties and biological assessment factors such as solubility (Bowman \& Sans 1983), bioconcentration factor (BCF; Gobas, et al. 1988), median lethal concentration (LC50), and median lethal dose (LD50; Hodson, et al. 
1988). Three computer programs (CLogP, MMP, and ACDLogP) and two estimation techniques based on molar volumes (Gobas, et al., 1988) and molecular surface areas (Smejtek, et al. 1996) are discussed in this work.

\section{Limitations of the Octanol-Water Membrane Model}

Besides scale differences, there are some significant differences between the octanolwater system and highly ordered biological lipid membrane-water membranes.

The bulk octanol phase used as a model for biomembrane phases must be electrically neutral, but it is well known that all membranes are electrically charged, and, in addition, that ceils produce and maintain transmembrane potentials of up to 60 $\mathrm{mV}$. Cells produce these potentials by creating imbalances between environmental and internal concentrations of ions such as calcium, sodium, and potassium using membrane proteins.

It is established (Smejtek, et al. 1993; Escher et al. 1996; Gobas, et al. 1988) that neutral compounds partition between octanol and water about the same as between lipids and water. However, ionic compounds have been shown to partition very differently in octanol water systems then in lipid membrane-water systems. The difference between $\log K_{O W}$ and $\log K_{L W}$ can be over several orders of magnitude, even at $\mathrm{pH}$ just one or more units above the pKa, (Smejtek, et al. 1993; Escher et al. 1996). However, data on octanol-water partition coefficients are very limited. In this work we measure the ionized and neutral partitioning of four halogenated phenols, 2,3,4,6-tetrachlorophenol, pentafluorophenol, pentachlorophenol, and 
pentabromophenol, and discuss the discrepancies between octanol-water and lipid membrane-water data.

\section{Purpose}

The objectives of this study are:

1) To test a model for the distribution of neutral and ionized halogenated phenols in an octanol-water system. The distribution of the four halogenated phenols 2,3,4,6-tetrachlorophenol, pentachlorophenol, pentafluorophenol, and pentabromophenol is determined using the "shake flask" method. The halogenated phenols PBP and PFP are studied since they have similar structures as PCP (Figure 3) and thus will be useful for objective 3 below.

2) To determine whether or not the octanol-water system is a viable model for predicting the partitioning of ionizable halophenols in a lipid membrane-water system. Octanol-water results from this study are compared with lipid membrane-water results obtained by other investigators, including 2,4,6-trichlorophenol and 2,3,4,5-tetrachlorophenol studied earlier (Schmidt 1995; Sieder 1995).

3) To evaluate, in the context of this study, two $\mathrm{K}_{\mathrm{OW}}$ prediction models based on solute physical properties. Using the results of this study, and results from other authors, two models based on solute molar volume and molecular surface area are discussed. 


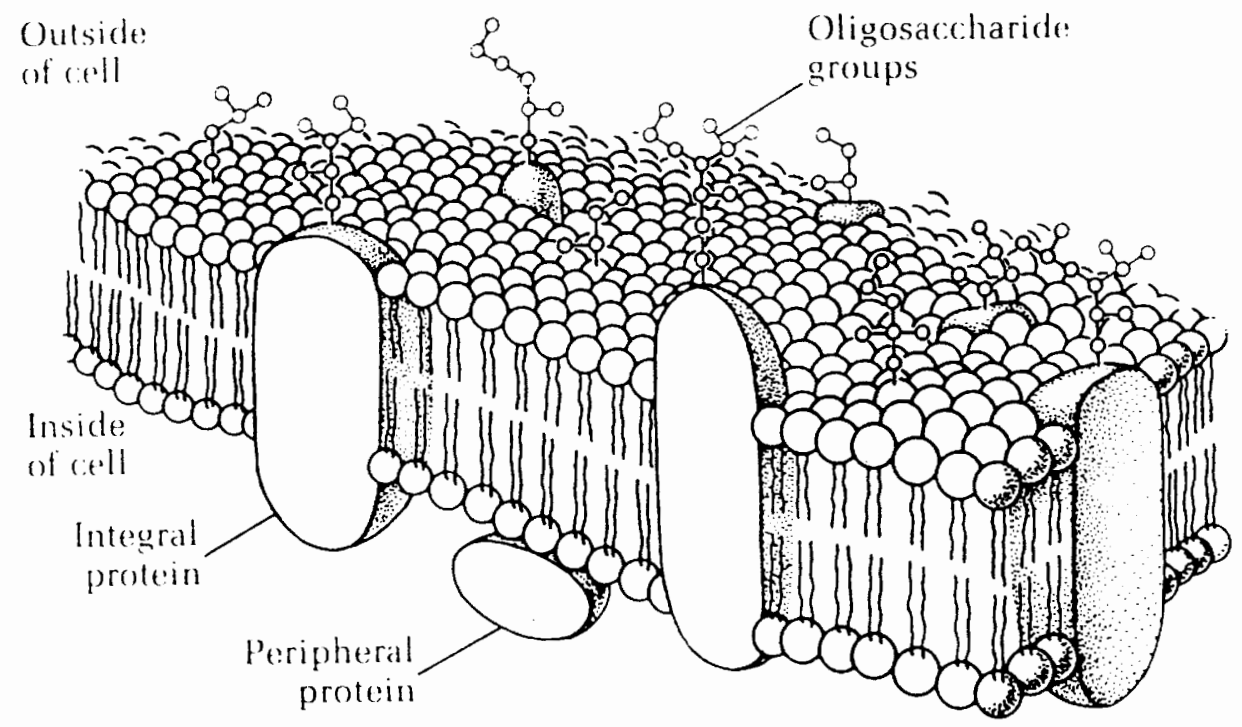

Figure 1. The fluid mosaic model of a biological membrane (Lehninger 1982). 

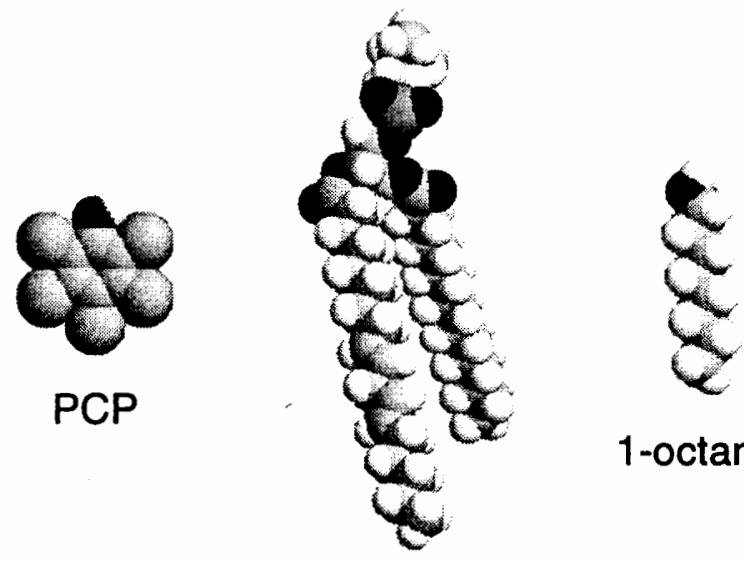

PCP

1-octanol

phosphatidylcholine

Figure 2. Space filling molecular models of pentachlorophenol, 1-octanol, and a typical lipid, phosphatidylcholine. All drawn approximately to the same scale.<smiles>Oc1c(Cl)cc(Cl)c(Cl)c1Cl</smiles>

2,3,4,6-TeCP (HA)<smiles>Oc1c(F)c(F)c(F)c(F)c1F</smiles>

PFP (HA)<smiles>Oc1c(Cl)c(Cl)c(Cl)c(Cl)c1Cl</smiles>

PCP (HA)<smiles>Oc1c(Br)c(Br)c(Br)c(Br)c1Br</smiles>

PBP (HA)

Figure 3. Molecular structure of the unionized halogenated phenols used in this study. From left to right: 2,3,4,6-tetrachlorophenol, pentafluorophenol, pentachlorophenol, and pentabromophenol. 


\section{Theory}

\section{Ideal Partitioning}

A solute distributes between two immiscible ideal solvents $\alpha$ and $\beta$ in a way that maximizes the entropy $S$ of the entire system. Assuming that the phases are at constant temperature $T$ and pressure $p$ at equilibrium, we can use the Gibb's thermodynamic potential or free energy to describe the system (Sieder 1995). The free energy $G_{\alpha}$ of the $\alpha$ phase is

$$
G_{\alpha}=U_{\alpha}-S_{\alpha} T+p V_{\alpha}
$$

Where $U_{\alpha}$ is the internal energy and $V_{\alpha}$ is the volume. A differential change from equilibrium in the free energy $d G_{\alpha}$ is

$$
d G_{\alpha}=d U_{\alpha}-S_{\alpha} d T-T d S_{\alpha}+p d V_{\alpha}+V_{\alpha} d p
$$

Since

$$
d U_{\alpha}=T d S_{\alpha}-p d V_{\alpha}+\mu_{\alpha} d N_{\alpha}
$$

the change in free energy becomes,

$$
d G_{\alpha}=\mu_{\alpha} d N_{\alpha}-S_{\alpha} d T+V_{\alpha} d p
$$

If the differential changes in the pressure and temperature are zero $(d p=d T=$ 0 ), the change in the Gibb's free energy is dependent only on the chemical potential $\mu_{\alpha}$ and the change in the number of particles $d N_{\alpha}$. Under these conditions, the chemical potential is given by 


$$
\mu_{\alpha}=\left(\frac{\partial G_{\alpha}}{\partial N_{\alpha}}\right)_{T, p}
$$

If $d p \neq 0$ and the differential change in the number of particles $d N_{\alpha}$ vanishes, equation 4 is then

$$
V_{\alpha}=\left(\frac{\partial G_{\alpha}}{\partial p}\right)_{T, N}
$$

Differentiating equation 5 with respect to pressure, $\partial / \partial p$, and using equation 6 leads to one of Maxwell's relations (Kittel and Kroemer 1980).

$$
\frac{\partial^{2} G_{\alpha}}{\partial N_{\alpha} \partial p}=\left(\frac{\partial V_{\alpha}}{\partial N_{\alpha}}\right)_{p, T}=\left(\frac{\partial \mu_{\alpha}}{\partial p}\right)_{N, T}
$$

Combined with the ideal gas law $p V=N R T$, equation 7 gives a relation between the chemical potential, the temperature, and the pressure

$$
\left(\frac{\partial}{\partial N_{\alpha}} \frac{N_{\alpha} R T}{p}\right)_{p, T}=\left(\frac{\partial \mu_{\alpha}}{\partial p}\right)_{N, T},
$$

or, with the constants of integration $p_{0}^{*}$ and $\mu_{\alpha 0}^{*}$,

$$
\mu_{\alpha}=R T \ln p / p_{0}^{*}+\mu_{\alpha 0}^{*}
$$

Since the concentration is defined as $c_{\alpha}=N_{\alpha} / V_{\alpha}$, the pressure can be written from the ideal gas law as $p=c_{\alpha} R T$. Now equation 8 becomes a relation between the chemical potential, the temperature, and the concentration of the solute in the $\alpha$-phase. With a similar relation for the $\beta$-phase, we arrive at the pair 


$$
\left\{\begin{array}{l}
\mu_{\alpha}=R T \ln c_{\alpha}+\left(\mu_{\alpha 0}^{*}-R T \ln c_{\alpha 0}^{*}\right)=R T \ln c_{\alpha}+\mu_{\alpha 0} \\
\mu_{\beta}=R T \ln c_{\beta}+\left(\mu_{\beta 0}^{*}-R T \ln c_{\beta 0}^{*}\right)=R T \ln c_{\beta}+\mu_{\beta 0}
\end{array}\right\} .
$$

Where $\mu_{\alpha 0}$ and $\mu_{\beta 0}$ are the chemical potentials of a standard solution. When the two phases are in contact and in equilibrium, their respective chemical potentials are equal. In this case, when the initial system is at equilibrium $\mu_{\alpha}=\mu_{\beta}$. We can use (9) to write

$$
R T \ln c_{\alpha}-\mu_{\alpha 0}=R T \ln c_{\beta}-\mu_{\beta 0}
$$

Rearranging terms leads to a relation between the ratio of the concentrations and the difference in the standard chemical potentials of the $\alpha$-phase and the $\beta$-phase. The ratio of the concentrations is constant and is called the partition coefficient $K_{\alpha \beta}$ or $P_{\alpha \beta}$.

$$
K_{\alpha \beta}=\frac{c_{\alpha}}{c_{\beta}}=e^{\left(\mu_{\alpha 0}-\mu_{\beta 0}\right) / R T}
$$

Given $K_{\alpha \beta}$, the concentration of one phase is easily determined by measuring the concentration of the second phase.

\section{Solutions of Two Species and the Distribution Function}

Analogous to the partition coefficient, the distribution $D$ of the neutral (HA) and ionized $\left(A^{-}\right)$species of a solute, such as halogenated phenols, between octanol and aqueous phases is given by 


$$
D=\frac{[H A]_{o c t}+\left[A^{-}\right]_{o c t}}{[H A]_{a q}+\left[A^{-}\right]_{a q}}
$$

Where the brackets $[\ldots]_{o c t}$ and $[\ldots]_{a q}$ denote octanol and aqueous concentrations, respectively. Equation 12 can be written as

$$
D=\frac{\frac{[H A]_{o c t}}{[H A]_{a q}}+\frac{\left[A^{-}\right]_{o c t}}{\left[A^{-}\right]_{a q}} \cdot \frac{\left[A^{-}\right]_{a q}\left[H^{+}\right]_{a q}}{[H A]_{a q}} \cdot \frac{1}{\left[H^{+}\right]_{a q}}}{1+\frac{\left[A^{-}\right]_{a q}\left[H^{+}\right]_{a q}}{[H A]_{a q}} \cdot \frac{1}{\left[H^{+}\right]_{a q}}}
$$

The acidity or $p H$ of the solution is defined as

$$
p H \equiv-\log \left[H^{+}\right]_{a q}
$$

and the dissociation constant $p K a$ is given by

$$
p K a \equiv-\log \frac{\left[H^{+}\right]_{a q}\left[A^{-}\right]_{a q}}{[H A]_{a q}}
$$

Here we define the distribution parameters $\mathrm{g}_{\mathrm{HA}}$ and $\mathrm{g}_{\mathrm{A}}$.

$$
g_{H A} \equiv \frac{[H A]_{o c t}}{[H A]_{a q}}, \quad g_{A} \equiv \frac{\left[A^{-}\right]_{o c t}}{\left[A^{-}\right]_{a q}}
$$

Using the definitions 14 through 17 in equation 13 gives

$$
D(p H)=\frac{g_{H A}+g_{A} 10^{p H-p K a}}{1+10^{p H-p K a}} .
$$

This is the distribution equation. It describes the $\mathrm{pH}$ dependent non-linear partitioning of an ionizable chemical, such as a halogenated phenol, between octanol and water phases at a given temperature. The distribution parameters are obtained by 
measuring the partition coefficient in the low $\mathrm{pH}$ limit for $\mathrm{g}_{\mathrm{HA}}$ and the high $\mathrm{pH}$ limit for $\mathrm{g}_{\mathrm{A}}$. The $p K a$ of a solute can be measured or obtained from the literature. It is sufficient to determine $g_{H A}$ and $g_{A}$ at $\mathrm{pH}$ limits of \pm 4 or more above or below the pKa. ${ }^{3}$

$$
K_{O W}(H A)=\lim _{p H \rightarrow-\infty} D=g_{H A}, \quad K_{O W}\left(A^{-}\right)=\lim _{p H \rightarrow \infty} D=g_{A}
$$

A form of the distribution equation has been successfully applied to other systems, including lipid membrane-water systems (Escher 1996).

The $\mathrm{pH}$ dependent octanol-water partitioning of four halogenated phenols, 2,3,4,6-tetrachlorophenol, pentafluorophenol, pentachlorophenol, pentabromophenol was measured and fit, by computer, to the distribution model above (18). The $p K a$ of each halophenol studied is available from the literature. Solute concentrations in the aqueous phase were measured using UV-Vis spectrophotometry.

\section{The Octanol and Aqueous Phases}

So far, we have only considered immiscible ideal bulk solutions. In the octanol-water system, the equilibrium concentration of octanol in water is 6.15 M (Bowman \& Sans 1983) and the concentration of octanol in water is about 0.01 M (Smejtek, et al. 1996). To further complicate matters, under our experimental conditions, octanol micelles are believed to form in the aqueous phase. These octanol emulsions create, in effect, a third phase. Halophenols absorbed by micelles contribute to the concentration

\footnotetext{
3 At $\mathrm{pH}=\mathrm{pKa} \pm 4$ or more, the ratio of ionized to neutral species is $10,000: 1(\mathrm{pKa}+4)$ or $1: 10,000$
} $(\mathrm{pKa}-4)$. 
measured in the aqueous phase. For halophenols with very low solubilities, errors introduced to the aqueous concentrations will be discussed. 


\section{UV-Vis Spectrophotometry}

Spectroscopy is based on the Bohr-Einstein relation between the energy $\Delta E$ and the frequency $v$ of light emitted (or absorbed) by a molecule when it translates between two discrete quantum states $E_{i}$.

$$
\Delta E=E_{2}-E_{1}=h c / \lambda=h v
$$

Where $h$ is Planck's constant $\left(6.626 \times 10^{-34} \mathrm{~J} \cdot \mathrm{s}\right.$ or $\left.9.532 \times 10^{-14} \mathrm{kcal} \cdot \mathrm{s} / \mathrm{mol}\right), c$ is the speed of light, and $\lambda$ is the wavelength of the emitted or absorbed light.

Ultraviolet-visible (UV-Vis) spectroscopy concerns the narrow band of the electromagnetic spectrum between the wavelengths $200 \mathrm{~nm}$ and $700 \mathrm{~nm}$. This region is of prime importance to chemistry because it encompasses transition energies between the electronic states, or, more precisely, transitions between the ground state $E_{o}$ and excited states $E_{i}$ (I transitions in Figure 4). Electrons in excited states generally ${ }^{4}$ decay rapidly to the ground state. Thus, electronic transitions from an excited state to a higher state is unlikely (II and III transitions in Figure 4).

Besides electronic transitions, photons are generally absorbed in transitions to higher vibrational and rotational states. UV-Vis Spectroscopy is only concerned with electronic and vibrational transition energies since the rotational modes of solutes are suppressed by solvent molecules.

For a diatomic model, vibrational modes of the connecting bond are described by the discrete energies $E_{v i}$ of the simple harmonic oscillator,

\footnotetext{
4 A population inversion occurs when the number of electrons in a long lived or metastable state of many identical molecules is greater than the number of electrons in a lower state. Lasers are produced by stimulating the rapid decay of a population inversion with photons.
} 


$$
E_{v i}=\hbar \omega_{o}\left(1 / 2+n_{v i}\right)
$$

Where $\omega_{0}$ is the natural frequency of the harmonic oscillator and $n_{v i}$ is a positive integer. The vibrational ground state energy $(n=0)$ of the harmonic oscillator is thus non-zero and equal to $E_{\mathrm{v} 0}=\frac{1}{2} \hbar \omega_{o}$. The energy emitted (or absorbed) during a vibrational transition is

$$
E_{\mathrm{v} 2}-E_{\mathrm{v} 1}=\Delta E_{\mathrm{v}}=\hbar \omega_{o} \Delta n_{\mathrm{v}}
$$

Combined with equation 21 , we can see that photons of total energy $\Delta E+\Delta E_{\mathrm{v}}$ can be emitted (or absorbed) by a diatomic molecule. A spectral line corresponding to energy emitted from a molecule by an electronic transition is thus split into equally spaced lines of varying intensities, (Figure 5). Electronic transitions between vibrational ground states are the most probable and thus the most spectrally intense. At spectrophotometric resolutions, electronic spectral lines split by vibrational modes appear as broadened bands.

More complex molecules, such as halogenated phenols with thirteen bonds, have more complicated electronic and vibrational states, but, in principle, behave as a system of linked harmonic oscillators. Larger molecules may also have different geometries dependent on the excited states. In addition, electronic transitions may favor transitions between vibrational states over the ground states (Perkampus 1992). 


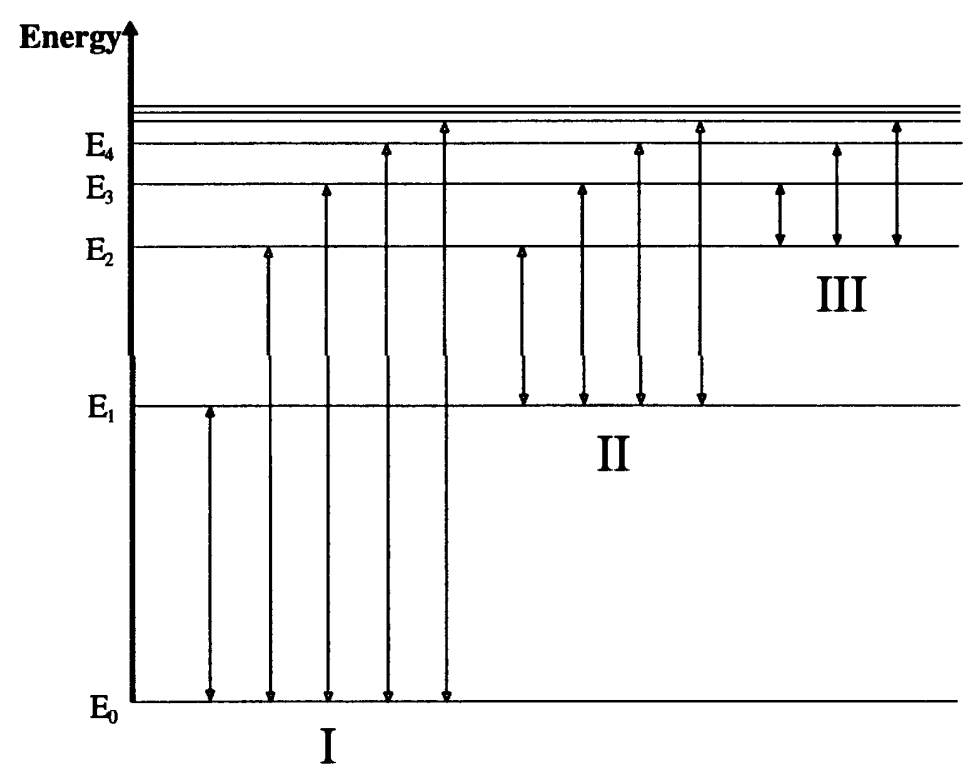

Figure 4. A simple energy level diagram showing possible transitions between singlet electronic states.
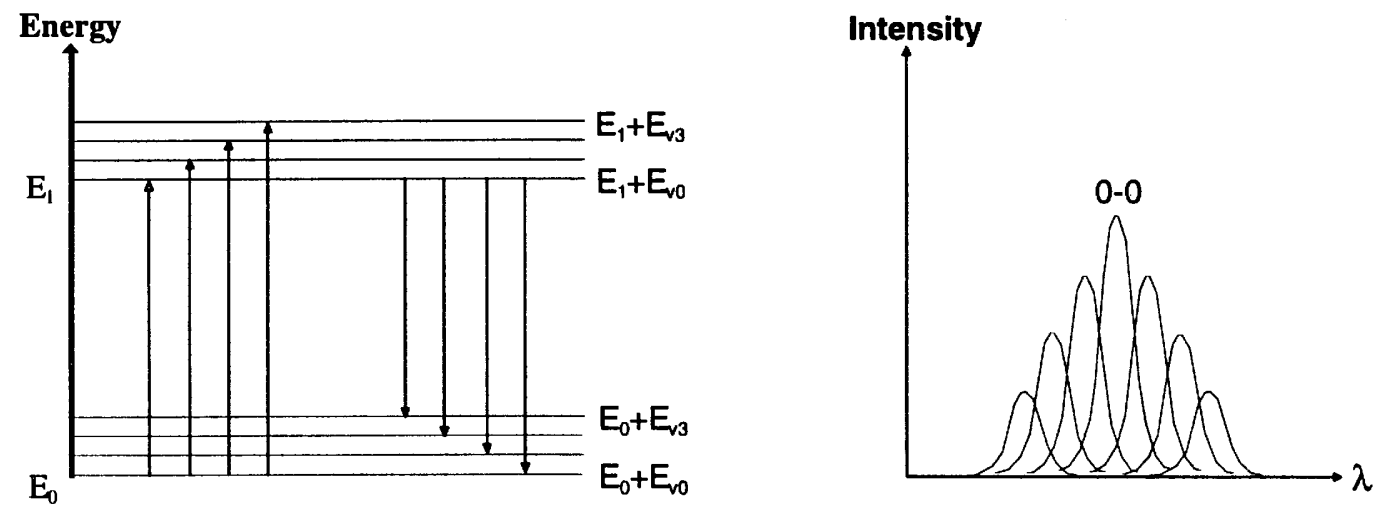

Figure 5. Left, Vibrational states superimposed on the ground and first excited electronic states. Right, A sketch showing the spectral broadening of radiation emitted from a diatomic molecule by vibrational transitions. The $E_{o}+E_{\mathrm{v} o}$ to $E_{l}+E_{\mathrm{v} o}$ transition is the most intense or probable. 


\section{Beer's Law and absorption spectroscopy}

In spectrophotometry, a monochromatic collimated beam of light of intensity $I_{0}$ is directed through a sample. Any light absorbed by the sample is re-radiated in random directions and of varying wavelengths. The emergent light of intensity $I\left(I_{0}\right.$ less absorbed light) is then measured by the spectrophotometer. The transmittance $T$ is defined as

$$
T=I / I_{0}
$$

and the absorbance $A$ is

$$
A=-\log T=\log \left(I_{0} / I\right)
$$

For dilute solutions, the absorbance is proportional to the path length $l$ (in $\mathrm{cm}$ ) of the sample and the concentration $c$,

$$
A=\varepsilon_{\lambda} c l .
$$

This relation is the Bouger-Lambert-Beer Law or simply, Beer's Law. The proportionality constant $\varepsilon_{\lambda}$ is the molar decadic extinction coefficient and has units of $\mathrm{cm}^{-1} \cdot \mathrm{mol}^{-1}$. The extinction coefficient is constant for a molecule at a specified wavelength that is usually chosen to correspond to a wavelength of a convenient peak in an absorbance spectrum. Given $\varepsilon_{\lambda}$ and $l$, we can measure $A$ and simply determine the concentration of a solute in solution.

To minimize stray and scattered light and absorption by other sources other than the solute molecules, the absorbance of a background or "blank" cell, without the 
compound of interest, is subtracted from the absorbance of the sample cell. For ultraviolet spectroscopy, quartz cuvettes are used to minimize the absorbance of the container walls. 


\section{DU-7 UV-VIS Spectrophotometer}

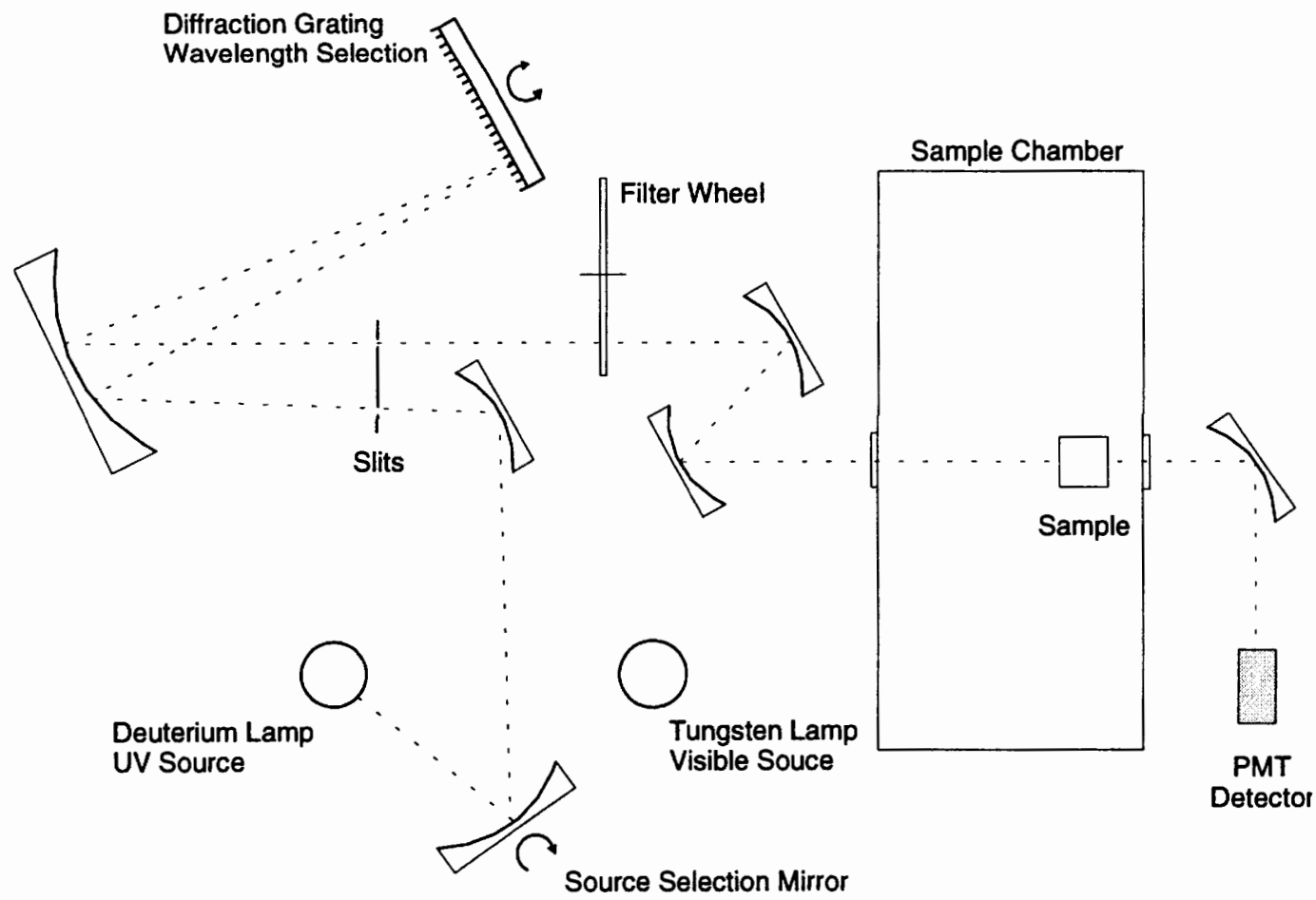

Figure 6. Schematic of a UV-Vis spectrophotometer.

\section{The DU-7 Spectrophotometer}

Figure 6 shows an internal diagram of the spectrophotometer used in our experiments. The visible $(315-700 \mathrm{~nm})$ source is a tungsten lamp. A deuterium lamp is used in the ultraviolet region (190-315 nm). Light is directed onto a diffraction grating that, depending on position, returns a selected wavelength through the slit via mirrors. The monochromatic light is then collimated by mirrors and directed through the sample chamber. Light transmitted by the sample cuvette is measured by the photomultiplier tube, the signal is digitized, and stored electronically. The position of the source selection mirror, position of the diffraction grating, and filter selection (if any) is all automated. 


\section{Materials and Methods}

\section{Chemicals}

2,3,4,6-tetrachlorophenol (2,3,4,6-TeCP) was obtained from TCI America (Portland, OR), pentachlorophenol (PCP), pentabromophenol (PBP), pentafluorophenol (PFP), and 99+\% spectroscopic grade 1-octanol were purchased from Aldrich Chemical Company, Inc. (Milwaukee, WI). The buffer components included: potassiumphosphate-dibasic-trihydrate $\left(\mathrm{K}_{2} \mathrm{HPO} 4 \cdot 3 \mathrm{H}_{2} \mathrm{O}\right)$ and boric acid $\left(\mathrm{HBO}_{3}\right)$ obtained from Mallinckrodt Chemicals (St. Louis, MO), and potassium citrate monohydrate $\left(\mathrm{K}_{3} \mathrm{C}_{6} \mathrm{H}_{5} \mathrm{O}_{7}\right)$ was obtained from Matheson Coleman \& Bell Manufacturing Chemists (Norwood, $\mathrm{OH})$. The other ionic compounds were: potassium chloride $(\mathrm{KCl})$ obtained from EM Science and potassium hydroxide (KOH) from Matheson Coleman $\&$ Bell. All aqueous solutions were prepared using $18.2+\mathrm{M} \Omega$ water. All chemicals were used without further purification.

\section{Instruments}

Chemical masses were determined using a Type $\mathrm{H} 16$ analytical balance, with an accuracy of $\pm 0.1 \mathrm{mg}$, manufactured by Mettler Instrument (Nightstown, NJ).

Acidity measurements were made using a Model $6072 \mathrm{pH}$ Meter, with a specified accuracy of \pm 0.01 , manufactured by Jenco Electronics Ltd., Taipei, Taiwan.

A DU-7 Spectrophotometer (manufactured by Beckman Industries, Fullerton, CA) with published tolerances of $\pm 0.5 \%$ absorbance and $\pm 0.5 \mathrm{~nm}$ wavelength was 
used for absorbance measurements. Ultraviolet absorbance of samples from the buffer phase were measured using $1.00 \pm 0.01 \mathrm{~cm}$ spectrophotometric cuvettes from Pyrocell Manufacturing Co., Inc., (Westwood, NJ), $0.10 \pm 0.01 \mathrm{~cm}$ and $5.00 \pm 0.05 \mathrm{~cm}$ cuvettes were obtained from Spectrocell Corp., (Oreland, PA). Absorbance measurements of the octanol phase were made using $1.00 \pm 0.01 \mathrm{~cm}$ cuvettes also obtained from Spectrocell Corp. The custom spectrophotometer control program was written by Piet O. Schmidt (1995).

Axum 4.0 for Windows, (Trimetrix, Inc.), was used on a 486DX PC for all computer aided data analysis. Molecular Modeling Pro (MMP) and Molecular Analysis Pro (MAP), purchased from Windowchem Software, were used to obtain molecular structure parameters. Two demos of other software packages, ACD (Advanced Chemical Development Inc.) and CLogP (Biobyte Inc.) were obtained from the publishers for evaluation, (see appendix).

\section{Preparation of the Octanol and Buffer Phases}

The aqueous buffer (KPCB) consists of $0.03 \mathrm{M}$ potassium chloride salt and a hundred times diluted stock solution of $0.2 \mathrm{M}$ potassium-phosphate, $0.2 \mathrm{M}$ boric acid, and 0.05 M potassium citrate. Both the octanol and buffer phases were presaturated with water and octanol, respectively, to control for volume and spectrophotometric effects. To presaturate the phases, buffer and octanol were added, at a volume ratio of 5 to 1 , to six $250 \mathrm{ml}$ polypropylene centrifugal bottles and shaken vigorously for 1 hour on a Model BB Wrist Action Shaker, (manufactured by Burrel Corp., Pittsburgh, PA). 
The bottles were then centrifuged at $1500 \mathrm{~g}$ for 1 hour in a IEC DPR-600 temperature controlled centrifuge (International Equipment Comp., Needham Heights, MA) to separate the octanol and water saturated phases. The octanol phase, which floats above the buffer phase, was then extracted from the surface with a $30 \mathrm{ml}$ glass syringe and transferred to a $500 \mathrm{ml}$ brown-glass bottle. The saturated buffer phase, (KPCB*), was extracted by pipette and stored in 1 liter brown-glass bottles. Approximately $5 \mathrm{ml}$ of each phase was discarded to avoid interfacial mixing. To minimize microbial contamination, buffer and saturated buffer were stored in the laboratory refrigerator.

\section{Extinction Coefficients}

For each phenol the extinction coefficient was measured for the ionized (A) form in saturated buffer. Three or four $20 \mathrm{mM}$ stock solutions of each phenol were produced by dissolving a few millimoles of dry chemical in $0.10 \mathrm{M} \mathrm{KOH}$ or, for TeCP, in buffer with a small amount of $\mathrm{KOH}$ added. Saturated buffer was not used for stock solutions since dissolved octanol precipitated out of the buffer and formed aggregates with undissolved phenol. Solutions of $\mathrm{KOH}$ were used since the ionized form of each chemical is far more soluble in water. Each solution was covered with aluminum foil and stirred by a magnetic stirrer for a few hours or more to completely dissolve the phenol.

Two to six $25 \mathrm{ml}$ test solutions were produced from each stock solution by diluting a few milliliters of stock with saturated buffer. Concentrations were selected to have expected absorbances between 0.1 and 2 , the most accurate absorbance range 
of the spectrophotometer.

The $\mathrm{pH}$ of each test solution was then measured. To completely ionize TeCP, $\left(99.99+\% \mathrm{~A}^{-}\right)$, each test solution was titrated with $\mathrm{KOH}$ to $\mathrm{pH}$ above 10 .

Concentrations changes due to volume changes were noted. Since the stock solutions of the other chemicals were prepared with $0.10 \mathrm{M} \mathrm{KOH}$ and test solutions had $\mathrm{pH}$ above 10 , the titration step was unnecessary.

A few milliliters of test solution were then added to a $1 \mathrm{~cm}$ sample cuvette and saturated buffer to a background cuvette. The surface of the optically matched ${ }^{5}$ cuvettes was wiped with chloroform and carefully polished with lens tissue before use. The spectrophotometer was repetitively calibrated to remove a discontinuity between the visible and ultra-violet spectra at $315 \mathrm{~nm}$. Once calibrated satisfactorily, the baseline of the spectrophotometer was stable for the remainder of the day. Each sample was scanned over a range from $200 \mathrm{~nm}$ to $370 \mathrm{~nm}$ five successive times. The "true" absorbance value is taken as the mean of absorbances measured at a selected peak from the five scans. One scan from each test solution was saved to disk. For measurements of new test solutions, the sample cuvette was rinsed at least three times with the next solution to be measured. Otherwise, the cuvettes were rinsed with distilled water and than ethanol and allowed to dry.

5 The absorbances of the same solution measured with optically matched cuvettes should be the same (within \pm 0.001 ). 


\section{Octanol-Water Partitioning}

The distribution of the ionized and neutral species of halogenated phenols was determined using the "shake-flask" method. A one hundred milliliter $20 \mathrm{mM}$ to 90 $\mathrm{mM}$ phenol solution was prepared in saturated octanol. The same phenol solution, with a few exceptions ${ }^{6}$, was used for all partitioning trials of each chemical. For each trial, $60 \mathrm{ml}$ of saturated buffer was titrated with $\mathrm{HCl}$ or $\mathrm{KOH}$ to a selected $\mathrm{pH}$ between 2.5 and 12.5. Twenty-five milliliters of the $\mathrm{pH}$-adjusted saturated buffer was then transferred by pipette to a $50 \mathrm{ml}$ glass centrifuge sample bottle. $25 \mathrm{ml}$ of the same buffer was also added to an identical background bottle. Five milliliters of the subject phenol solution was added by $5 \mathrm{ml}$ pipette to the sample bottle. Five milliliters of saturated octanol were added, in the same fashion, to the background bottle. The bottles were then capped and shaken for 1 hour at an approximately $45^{\circ}$ angle on the wrist action shaker. After shaking, the bottles were centrifuged at $1500 \mathrm{~g}$ for 1 hour in a Safeguard Centrifuge (Clay-Adams, Inc., Parsippany, NJ) and allowed to cool for at least 30 minutes.

The octanol phases from both sample and background bottles were then carefully extracted by pipette and discarded. Twenty milliliters of the sample buffer solution were extracted and transferred to a $50 \mathrm{ml}$ beaker containing a magnetic stirring rod. The final experimental $\mathrm{pH}$ was measured using an electrode carefully

\footnotetext{
${ }^{6}$ The concentration of phenol in very acidic solutions is so low that, for better spectrophotometric results, a second, three to five times concentrated, initial octanol phase solution was necessary for TeCP and PCP. Unfortunately, this option is not available in the PBP experiments since the initial $90 \mathrm{mM}$ solution is nearly saturated.
} 
dried with the tip of piece of tissue. To avoid $\mathrm{pH}$ changes due to dissolved atmospheric $\mathrm{CO}_{2}$, (from carbonic acid, $\mathrm{H}_{2} \mathrm{CO}_{3}$, and its derivatives), the $\mathrm{pH}$ was quickly measured after removal of the octanol phase. Samples with a low final $\mathrm{pH}$ were titrated above $\mathrm{pH} 10$ with $0.10 \mathrm{M} \mathrm{KOH}$. Changes in the phenol concentration due to the added $\mathrm{KOH}$ solution are corrected for in the final measurements.

Sample and background solutions were added, depending on the concentration, to a pair of $5.00,1.00$, or $0.10 \mathrm{~cm}$ cuvettes. As in the previous section, the absorbance of each sample was measured five successive times with the spectrophotometer and the scans saved to disk. The "true" absorbance is taken from the mean of the five experimental measurements. 


\section{Results}

\section{Extinction Coefficients}

We have determined the extinction coefficient for the ionized species of each halogenated phenol dissolved in octanol-saturated buffer. The absorbances of 14 to 18 concentrations of each phenol were measured and the individually calculated extinction coefficients are given in Tables 1, 2, 3, and 4. The plots of Figures 7, 9, 11, and 13 show the linear relationship between absorbance and concentration as described by the Beer-Lambert law (26), where the slope of each plot is given by the respective phenol's extinction coefficient. Sample absorbance spectra of each ionized halogen substituted phenol are shown in Figures 8, 11, 14, and 17. For reference, sample absorbance spectra for neutral halogen substituted phenols are shown in Figures $9,12,15$, and 18.

The extinction coefficients for the halogenated phenols are $5058 \pm 32$ $\mathrm{cm}^{-1} \cdot \mathrm{mol}^{-1}$ at $316.0 \mathrm{~nm}(2,3,4,6-\mathrm{TeCP}), 1285 \pm 7 \mathrm{~cm}^{-1} \cdot \mathrm{mol}^{-1}$ at $266.5 \mathrm{~nm}(\mathrm{PFP}), 5031 \pm$ $30 \mathrm{~cm}^{-1} \cdot \mathrm{mol}^{-1}$ at $319.5 \mathrm{~nm}(\mathrm{PCP})$, and $5770 \pm 29 \mathrm{~cm}^{-1} \cdot \mathrm{mol}^{-1}$ at $314.5 \mathrm{~nm}$ (PBP). No measurable difference is apparent between the extinction coefficients of phenols dissolved in octanol saturated buffer and phenols dissolved in unsaturated buffer. 


\section{Error analysis of extinction coefficients}

To determine the final uncertainty of the extinction coefficients, we consider the contribution of errors from each experimental step, (errors in mass, pipette volume, etc.). We can estimate this uncertainty by considering the error propagation equation (Bevington 1969).

$$
\sigma_{x}^{2} \cong \sigma_{u}^{2}\left(\frac{\delta x}{\delta u}\right)^{2}+\sigma_{v}^{2}\left(\frac{\delta x}{\delta v}\right)^{2}+\ldots+2 \sigma_{u v}^{2}\left(\frac{\delta x}{\delta v}\right)\left(\frac{\delta x}{\delta v}\right)+\ldots
$$

Neglecting the covariant terms leads to:

$$
\sigma_{x}^{2} \cong \sigma_{u}^{2}\left(\frac{\delta x}{\delta u}\right)^{2}+\sigma_{v}^{2}\left(\frac{\delta x}{\delta v}\right)^{2}
$$

Expanding the Beer-Lambert law (26) in the same fashion gives the variance of the extinction coefficient:

$$
\sigma_{\varepsilon}^{2} \cong \sigma_{C}^{2}\left(\frac{\delta}{\delta C} \frac{C l}{A}\right)^{2}+\sigma_{l}^{2}\left(\frac{\delta}{\delta l} \frac{C l}{A}\right)^{2}+\sigma_{A}^{2}\left(\frac{\delta}{\delta A} \frac{C l}{A}\right)^{2}
$$

Where the standard deviation of the concentration $\sigma_{C}$ was determined, in a similar fashion, to be approximately $1 \%$. The uncertainty in the path length between two $1 \mathrm{~cm}$ cuvettes $\sigma_{l}$ is about $0.014 \mathrm{~cm}$. And the standard deviation of the absorbance, $\sigma_{\mathrm{A}}$, is given by:

$$
\sigma_{A}^{2} \cong \sigma_{A_{A n t}}^{2}+\delta_{A_{E F}}^{2}
$$

Where the instrumental error $\sigma_{A_{l n t}}$ is approximately $0.5 \%$ and the mean deviation of 
each measurement $\delta_{A_{E \varphi}}$ is about 0.002 .

The mean extinction coefficients are given by:

$$
\varepsilon_{\text {final }} \cong \frac{\sum_{i}\left(\varepsilon_{i} / \sigma_{i}^{2}\right)}{\sum_{i}\left(1 / \sigma_{i}^{2}\right)}
$$

And the variance of each mean extinction coefficient is:

$$
\sigma_{\varepsilon}^{2} \cong \frac{1}{\sum\left(1 / \sigma_{i}^{2}\right)}
$$




\begin{tabular}{|c|c|c|c|c|c|c|}
\hline Stock & Solute & $\mathrm{pH}$ & $\mathrm{C}(\mu \mathrm{M})$ & $\mathrm{A}$ & $\varepsilon\left(\mathrm{cm}^{-1} \cdot \mathrm{M}^{-1}\right)$ & $\sigma_{\varepsilon}\left(\mathrm{cm}^{-1} \cdot \mathrm{M}^{-1}\right)$ \\
\hline $0.985 \mathrm{mM}$ & KCPB $^{*}$ & 10.04 & 87.3 & 0.437 & 4997 & 190 \\
\hline & KCPB $^{*}$ & 10.11 & 174 & 0.897 & 5158 & 140 \\
\hline & KCPB & 10.19 & 39.0 & 0.194 & 4965 & 345 \\
\hline & KCPB & 10.15 & 253 & 1.294 & 5113 & 118 \\
\hline & KCPB $^{*}$ & 10.15 & 291 & 1.477 & 5071 & 124 \\
\hline & KCPB $^{*}$ & 10.19 & 137 & 0.697 & 5082 & 136 \\
\hline & KCPB $^{*}$ & 10.02 & 394 & 1.953 & 5016 & 115 \\
\hline & KCPB $^{*}$ & 10.01 & 464 & 2.286 & 4924 & 112 \\
\hline & KCPB $^{*}$ & 10.14 & 483 & 2.456 & 5079 & 114 \\
\hline $0.8125 \mathrm{mM}$ & KCPB $^{*}$ & 10.15 & 160 & 0.855 & 5326 & 187 \\
\hline & KCPB $^{*}$ & 10.17 & 257 & 1.345 & 5238 & 169 \\
\hline & KCPB $^{*}$ & 10.11 & 193 & 0.946 & 4907 & 167 \\
\hline & KCPB & 10.17 & 419 & 2.081 & 4964 & 110 \\
\hline & KCPB & 10.21 & 321 & 1.636 & 5095 & 115 \\
\hline & KCPB $^{*}$ & 10.18 & 96.0 & 0.487 & 5073 & 140 \\
\hline & KCPB & 10.16 & 198 & 1.006 & 5067 & 111 \\
\hline & KCPB & 10.14 & 398 & 2.013 & 5044 & 108 \\
\hline
\end{tabular}

Table 1. Experimental extinction coefficients for ionized 2,3,4,6-TeCP in octanol-saturated buffer at $\lambda=316.0 \mathrm{~nm}$. The mean extinction coefficient, $\varepsilon$, is $5058 \pm 32 \mathrm{~cm}^{-1} \cdot \mathrm{M}^{-1}$. 


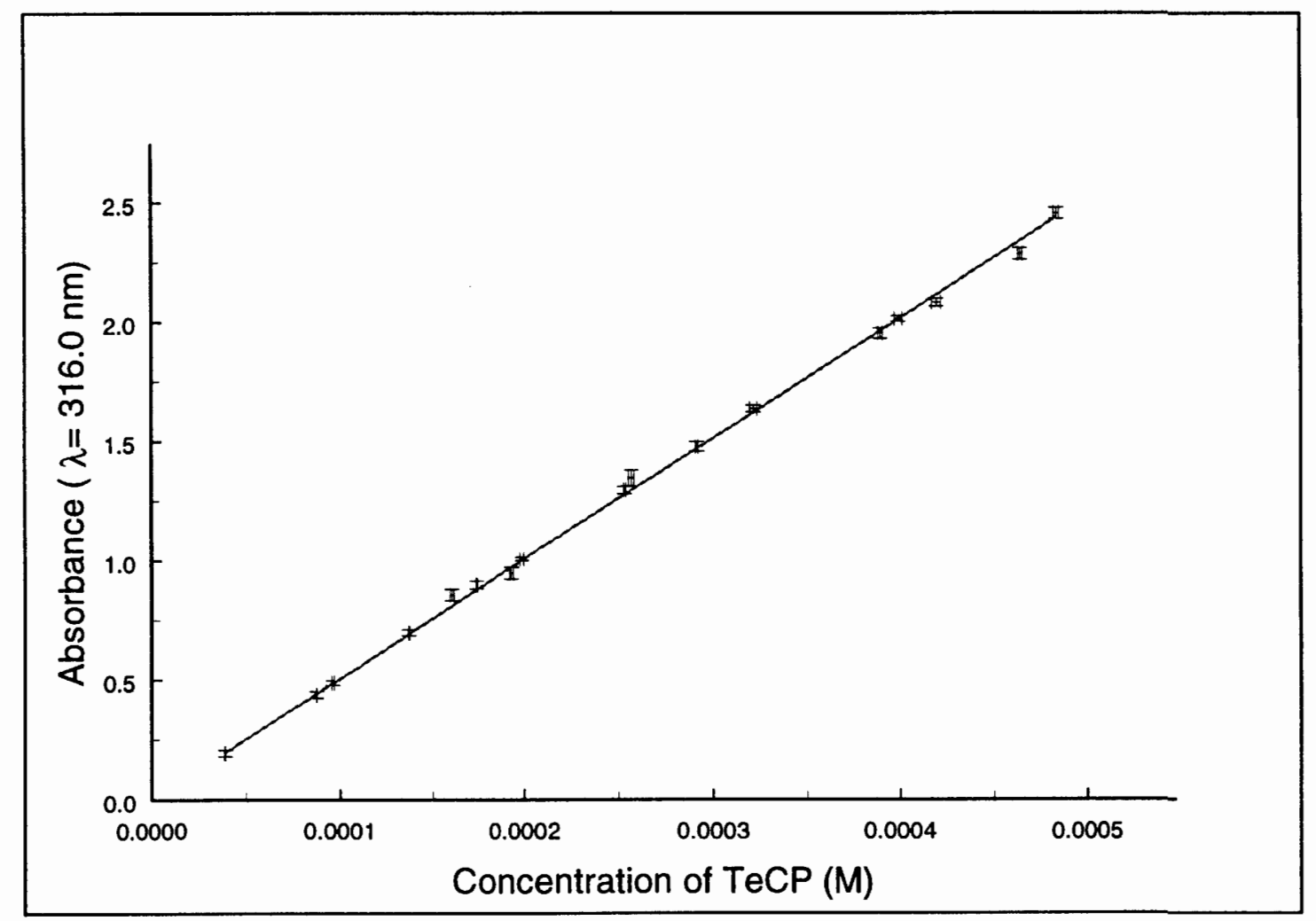

Figure 7. Absorbance versus concentration of ionized 2,3,4,6-TeCP at $\lambda=316.0 \mathrm{~nm}$. Sets of vertical and horizontal error bars enclose data points. The slope is the extinction coefficient. 


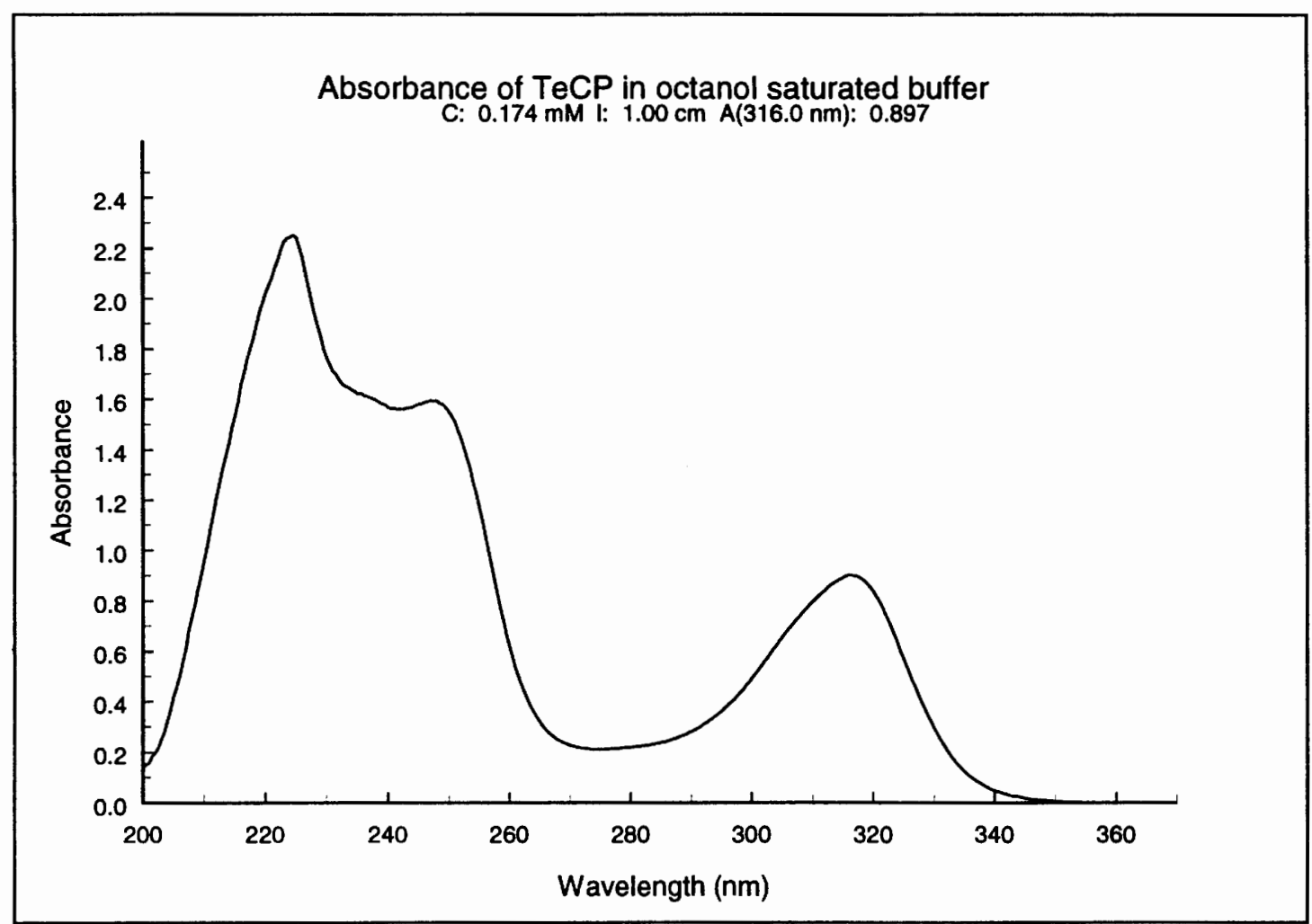

Figure 8. A typical absorbance spectrum of ionized 2,3,4,6-TeCP. The peak of interest is on the right, centered about $316 \mathrm{~nm}$. 


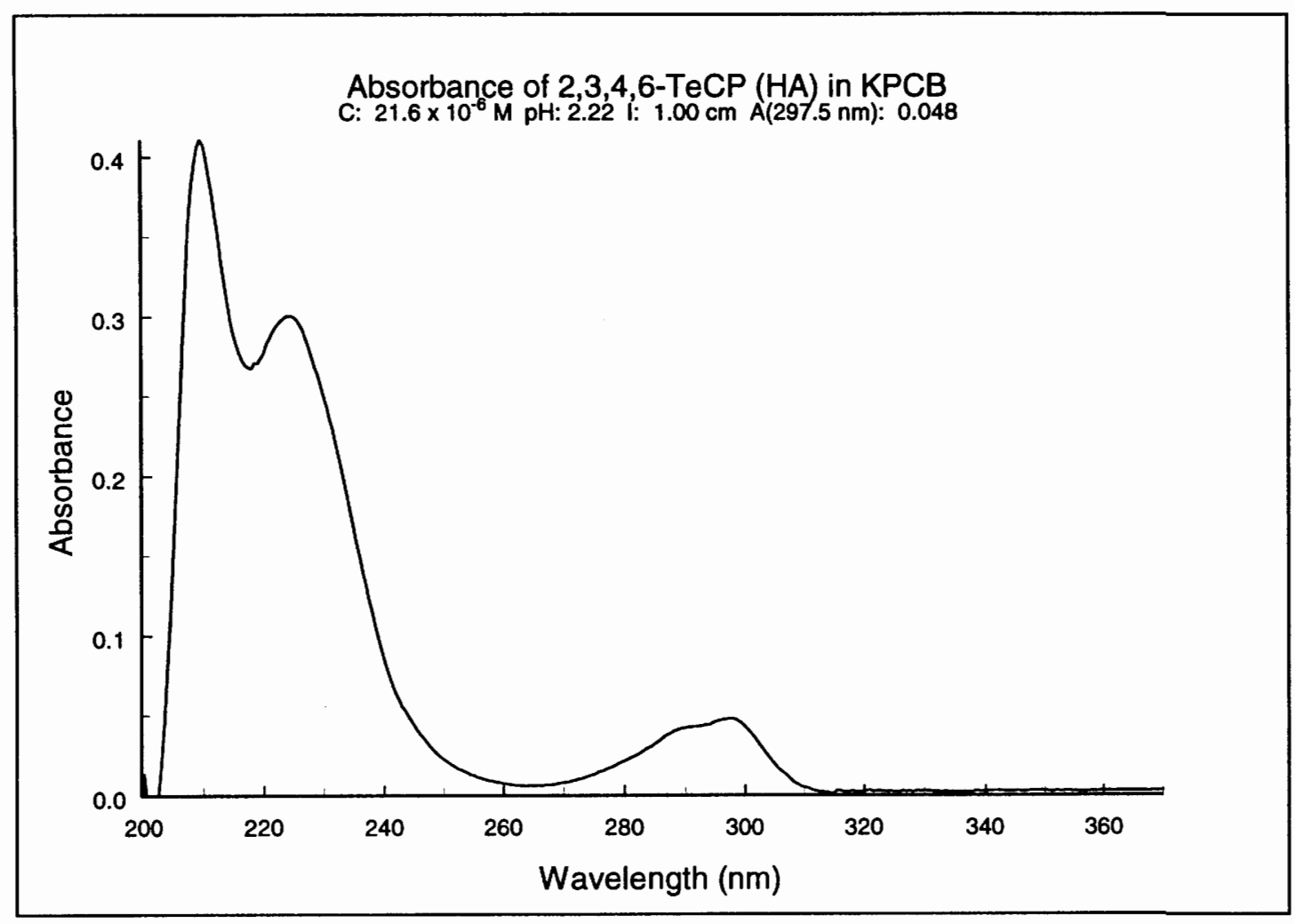

Figure 9. Absorbance spectrum of unionized 2,3,4,6-TeCP in unsaturated buffer. Due to the low solubility of the neutral species, the concentration is lower than the sample used in the previous spectrum (thus, the lower absorbances of the peaks). 


\begin{tabular}{|c|c|c|c|c|c|c|}
\hline Stock & Solute & $\mathrm{pH}$ & $\mathrm{C}(\mu \mathrm{M})$ & $\mathrm{A}$ & $\varepsilon\left(\mathrm{cm}^{-1} \cdot \mathrm{M}^{-1}\right)$ & $\sigma_{\varepsilon}\left(\mathrm{cm}^{-1} \cdot \mathrm{M}^{-1}\right)$ \\
\hline $7.575 \mathrm{mM}$ & KCPB $^{*}$ & 11.64 & 303.0 & 0.387 & 1277 & 30 \\
\hline & KCPB $^{*}$ & 12.01 & 606.0 & 0.768 & 1267 & 27 \\
\hline & KCPB $^{*}$ & 12.24 & 909.0 & 1.166 & 1283 & 27 \\
\hline & KCPB $^{*}$ & 12.40 & 1202 & 1.543 & 1284 & 27 \\
\hline & KCPB $^{*}$ & 11.46 & 227.3 & 0.293 & 1291 & 30 \\
\hline $6.676 \mathrm{mM}$ & KCPB $^{*}$ & 11.62 & 267.0 & 0.343 & 1285 & 27 \\
\hline & KCPB $^{*}$ & 12.07 & 534.0 & 0.680 & 1273 & 28 \\
\hline & KCPB $^{*}$ & 12.28 & 801.1 & 1.038 & 1296 & 27 \\
\hline & KCPB $^{*}$ & 12.44 & 1068 & 1.384 & 1296 & 32 \\
\hline & KCPB $^{*}$ & 12.51 & 1122 & 1.447 & 1290 & 31 \\
\hline $6.982 \mathrm{mM}$ & KCPB $^{*}$ & 11.51 & 223.4 & 0.287 & 1284 & 27 \\
\hline & KCPB $^{*}$ & 11.64 & 265.3 & 0.349 & 1315 & 27 \\
\hline & KCPB $^{*}$ & 12.38 & 977.5 & 1.264 & 1293 & 33 \\
\hline & KCPB $^{*}$ & 12.48 & 1117 & 1.438 & 1287 & 27 \\
\hline & KCPB $^{*}$ & 12.10 & 558.6 & 0.709 & 1269 & 27 \\
\hline
\end{tabular}

Table 2. Experimental extinction coefficients for ionized pentafluorophenol in octanol-saturated buffer at $\lambda=266.5 \mathrm{~nm}$. The mean extinction coefficient, $\varepsilon$, is $1285 \pm 7 \mathrm{~cm}^{-1} \cdot \mathrm{M}^{-1}$. 


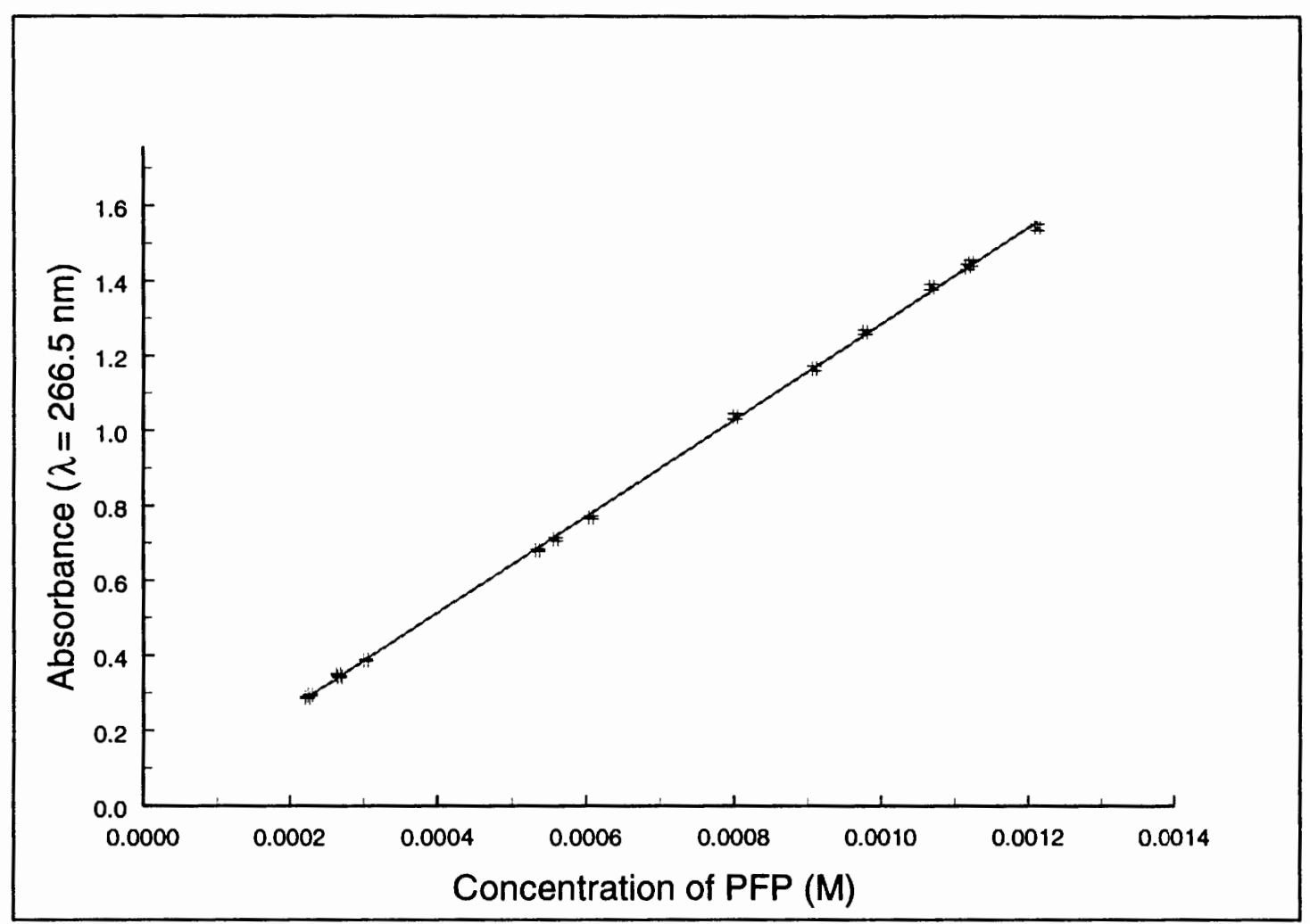

Figure 10. Absorbance versus concentration of ionized PFP at $\lambda=266.5 \mathrm{~nm}$. Sets of vertical and horizontal error bars enclose data points. The slope is the extinction coefficient. 


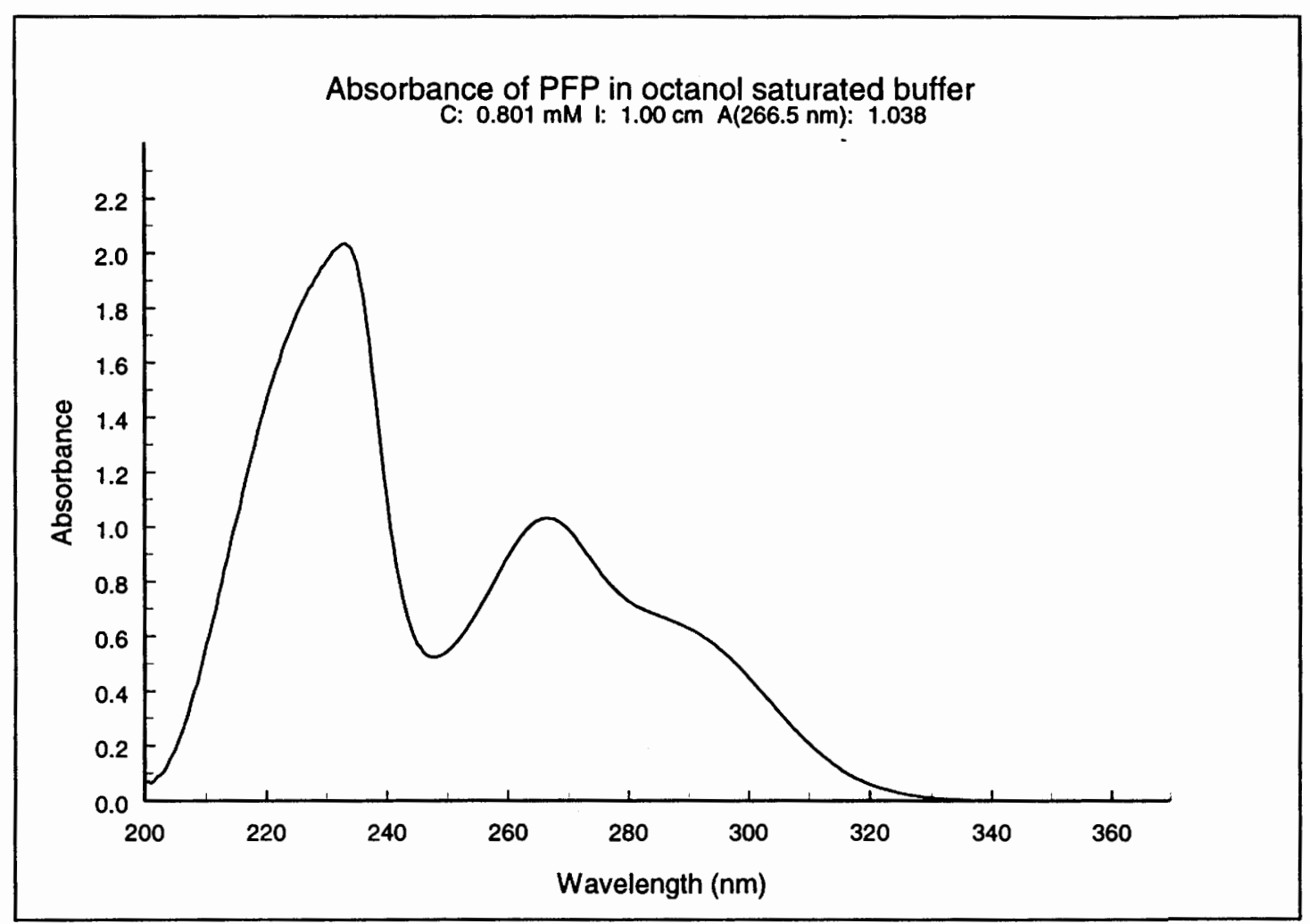

Figure 11. A typical absorbance spectrum of ionized PFP. The peak of interest is on the right, centered about $266.5 \mathrm{~nm}$. 


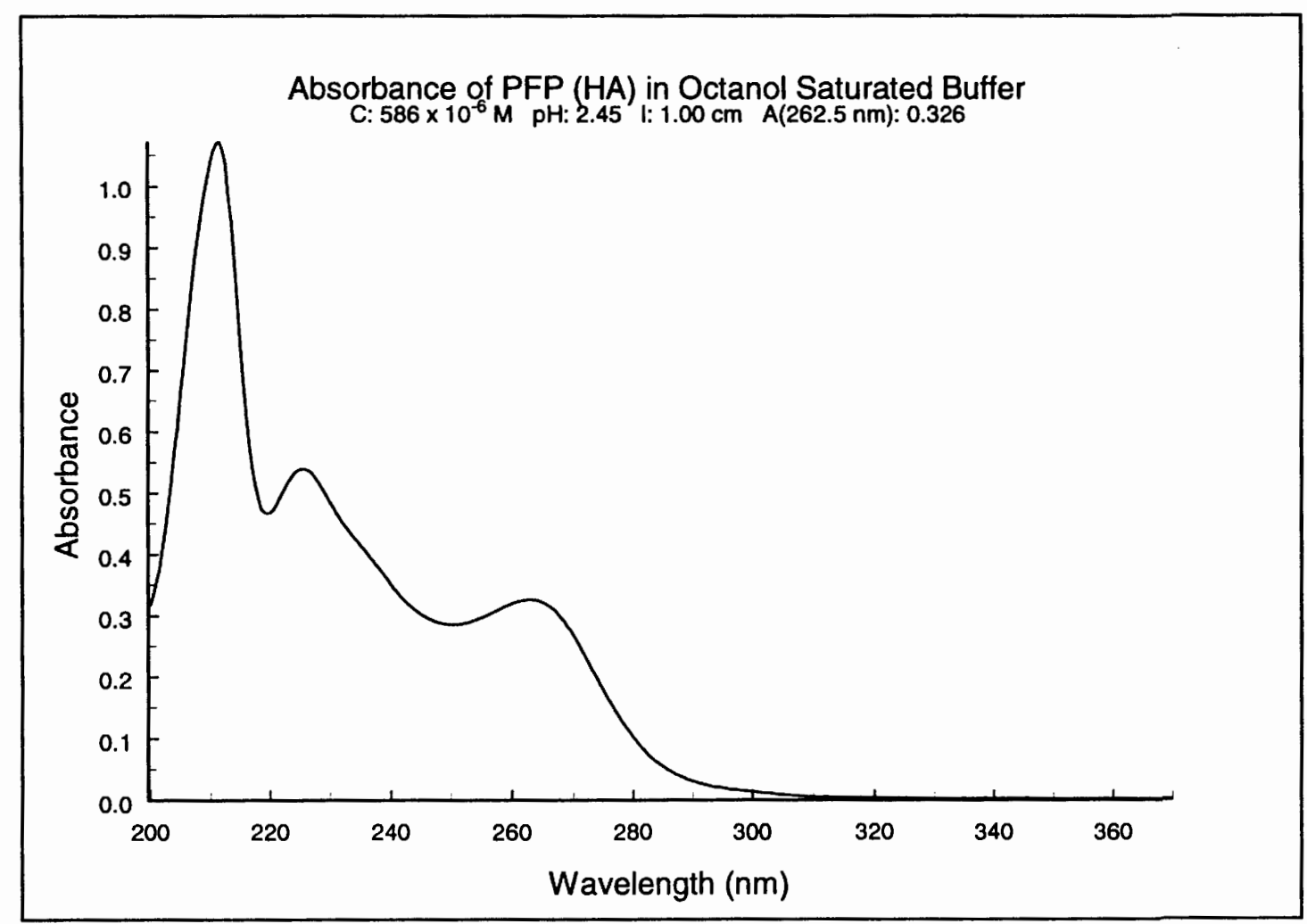

Figure 12. Absorbance spectrum of unionized PFP in octanol saturated buffer. 


\begin{tabular}{|c|l|c|c|c|c|c|}
\hline Stock & Solute & $\mathrm{pH}$ & $\mathrm{C}(\mu \mathrm{M})$ & $\mathrm{A}$ & $\varepsilon\left(\mathrm{cm}^{-1} \cdot \mathrm{M}^{-1}\right)$ & $\sigma_{\varepsilon}\left(\mathrm{cm}^{-1} \cdot \mathrm{M}^{-1}\right)$ \\
\hline $0.7397 \mathrm{mM}$ & KCPB $^{*}$ & 10.00 & 146.0 & 0.715 & 4900 & 106 \\
\hline & KCPB $^{*}$ & 10.12 & 207.1 & 1.026 & 4954 & 110 \\
\hline & KCPB $^{*}$ & 10.05 & 264.0 & 1.308 & 4955 & 109 \\
\hline & KCPB $^{*}$ & 10.00 & 323.5 & 1.608 & 4971 & 107 \\
\hline & KCPB $^{*}$ & 10.17 & 87.70 & 0.425 & 4846 & 106 \\
\hline & KCPB $^{*}$ & 9.99 & 117.1 & 0.584 & 4987 & 107 \\
\hline $3.000 \mathrm{mM}$ & KCPB $^{*}$ & 10.02 & 358.0 & 1.841 & 5142 & 109 \\
\hline & KCPB $^{*}$ & 10.24 & 178.0 & 0.911 & 5117 & 112 \\
\hline & KCPB $^{*}$ & 11.30 & 118.0 & 0.609 & 5161 & 121 \\
\hline & KCPB $^{*}$ & 10.44 & 238.0 & 1.218 & 5118 & 110 \\
\hline $5.000 \mathrm{mM}$ & KCPB $^{*}$ & 11.45 & 300.0 & 1.527 & 5090 & 112 \\
\hline & KCPB $^{*}$ & 11.18 & 200.0 & 1.009 & 5045 & 118 \\
\hline & KCPB $^{*}$ & 10.91 & 100.0 & 0.514 & 5140 & 149 \\
\hline & KCPB $^{*}$ & 12.18 & 400.0 & 2.034 & 5085 & 110 \\
\hline
\end{tabular}

Table 3. Experimental extinction coefficients for ionized pentachlorophenol in octanol-saturated buffer at $\lambda=319.5$. The mean extinction coefficient, $\varepsilon$, is $5031 \pm 30 \mathrm{~cm}^{-1} \cdot \mathrm{M}^{-1}$. 


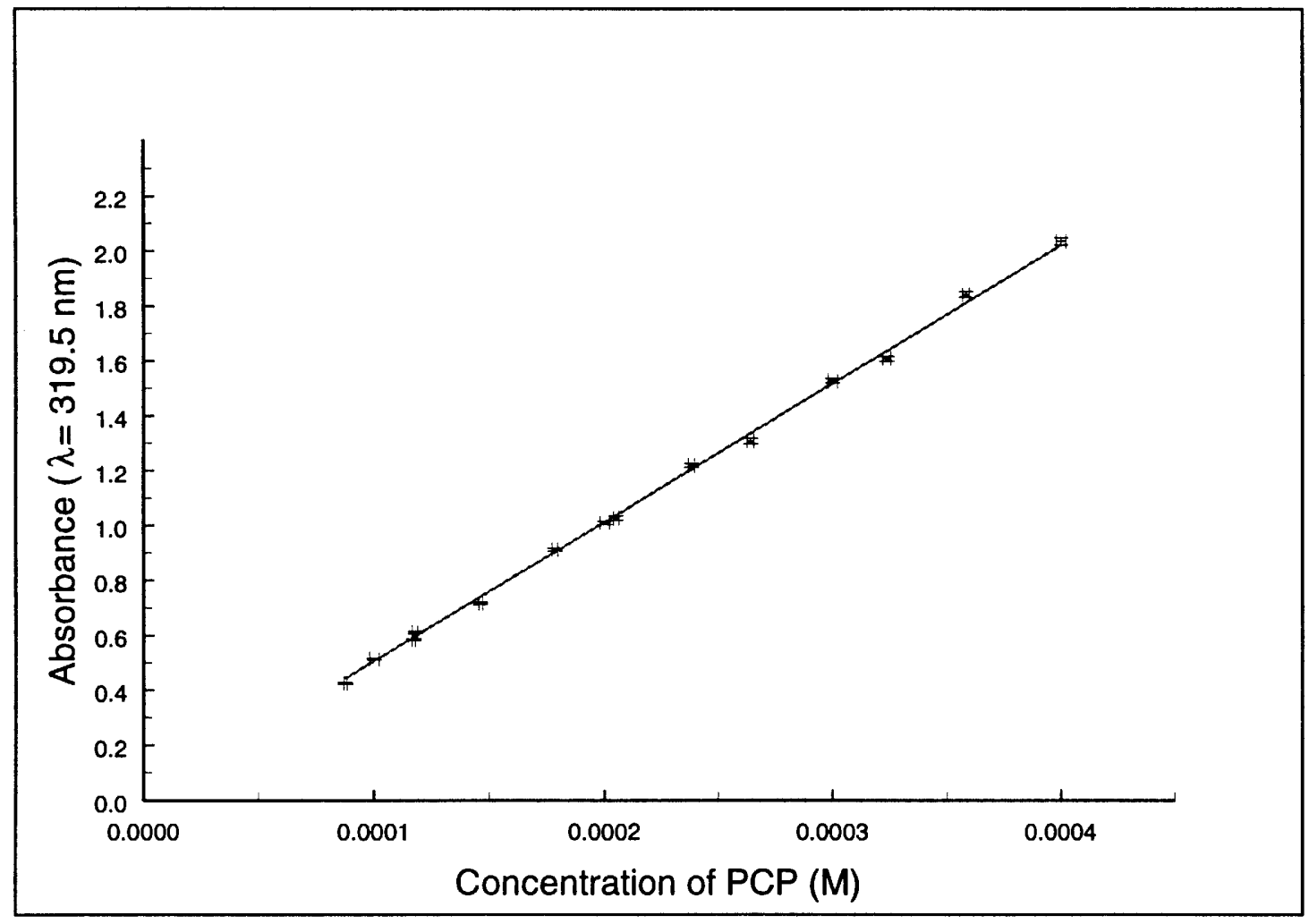

Figure 13. Absorbance versus concentration of ionized PCP at $\lambda=319.5 \mathrm{~nm}$. Sets of vertical and horizontal error bars enclose data points. The slope is the extinction coefficient. 


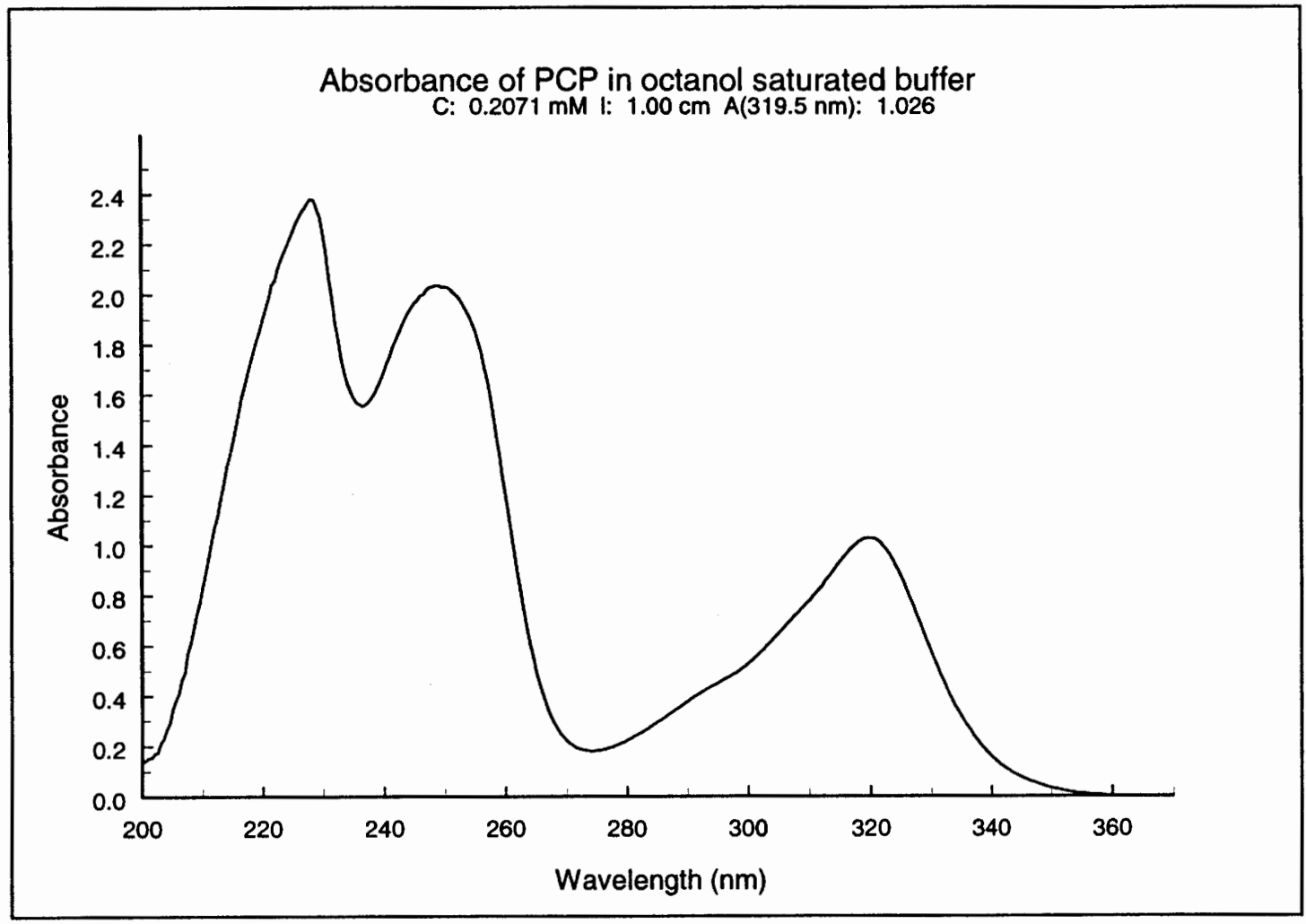

Figure 14. A typical absorbance spectrum of ionized PCP. The peak of interest is on the right, centered about $319.5 \mathrm{~nm}$. 


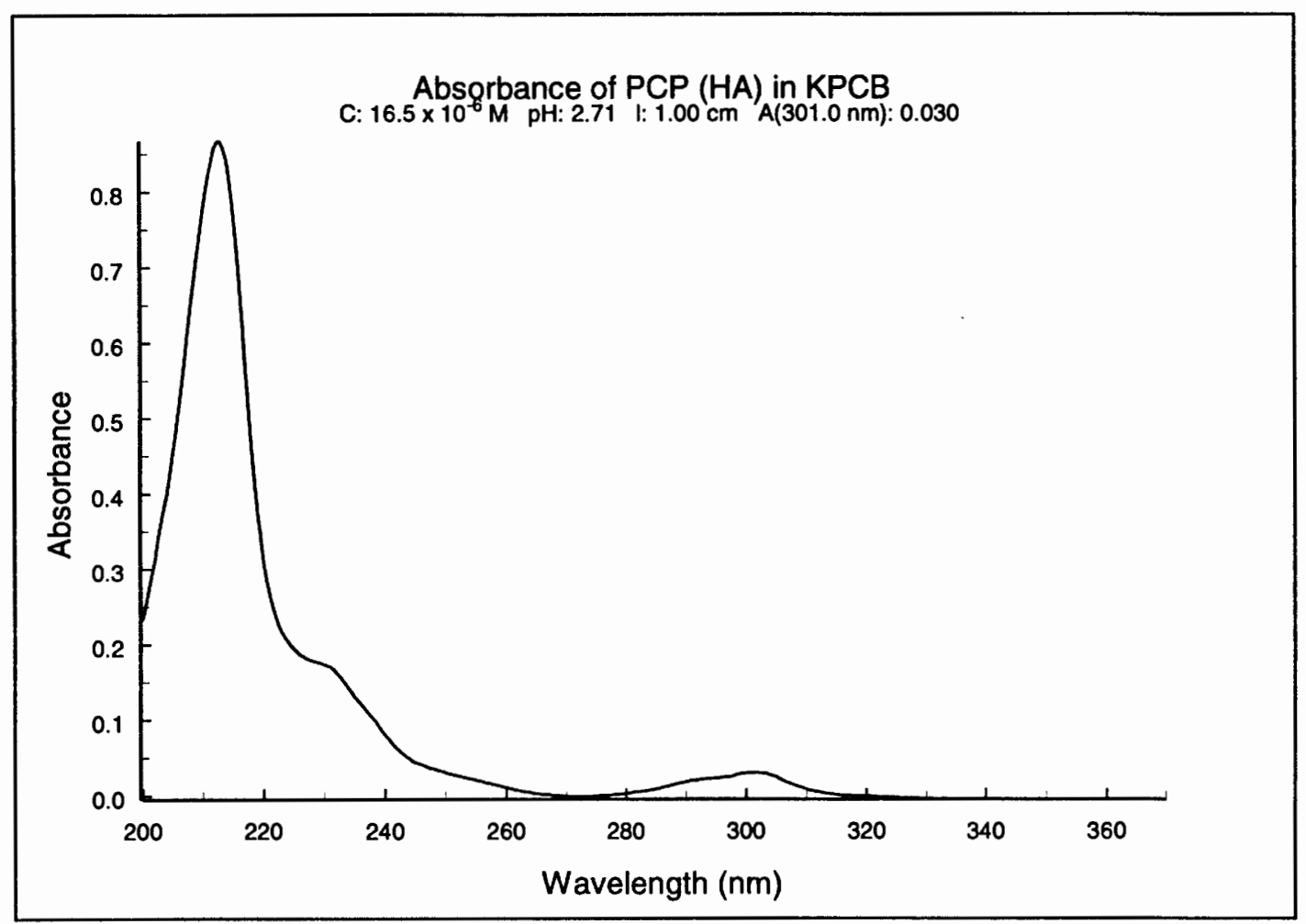

Figure 15. Absorbance spectrum of unionized PCP in unsaturated buffer. Due to the low solubility of the neutral species, the concentration is lower than the sample used in the previous spectrum (thus, the lower absorbances of the peaks). 


\begin{tabular}{|c|c|c|c|c|c|c|}
\hline Stock & Solute & $\mathrm{pH}$ & $\mathrm{C}(\mu \mathrm{M})$ & $\mathrm{A}$ & $\varepsilon\left(\mathrm{cm}^{-1} \cdot \mathrm{M}^{-1}\right)$ & $\sigma_{\varepsilon}\left(\mathrm{cm}^{-1} \cdot \mathrm{M}^{-1}\right)$ \\
\hline \multirow[t]{6}{*}{$2.000 \mathrm{mM}$} & KCPB* & 11.65 & 80.0 & 0.465 & 5815 & 136 \\
\hline & KCPB* & 12.14 & 160 & 0.934 & 5838 & 125 \\
\hline & KCPB* & 12.36 & 240 & 1.397 & 5823 & 122 \\
\hline & KCPB* & 12.53 & 320 & 1.838 & 5744 & 120 \\
\hline & KCPB* & 12.63 & 380 & 2.179 & 5735 & 120 \\
\hline & KCPB* & 12.21 & 200 & 1.162 & 5809 & 122 \\
\hline \multirow[t]{6}{*}{$1.000 \mathrm{mM}$} & KCPB* & 11.99 & 80.0 & 0.466 & 5825 & 126 \\
\hline & KCPB* & 12.22 & 120 & 0.685 & 5710 & 121 \\
\hline & KCPB* & 12.36 & 160 & 0.923 & 5768 & 121 \\
\hline & KCPB* & 12.14 & 100 & 0.572 & 5716 & 123 \\
\hline & KCPB* & 12.31 & 140 & 0.799 & 5709 & 120 \\
\hline & KCPB* & 12.45 & 180 & 1.046 & 5812 & 121 \\
\hline \multirow[t]{6}{*}{$1.250 \mathrm{mM}$} & KCPB* & 11.75 & 50.0 & 0.286 & 5720 & 135 \\
\hline & KCPB* & 12.17 & 100 & 0.573 & 5728 & 123 \\
\hline & KCPB* & 12.02 & 125 & 0.721 & 5766 & 122 \\
\hline & KCPB* & 12.15 & 150 & 0.866 & 5771 & 121 \\
\hline & KCPB* & 12.22 & 175 & 1.019 & 5821 & 122 \\
\hline & KCPB* & 12.33 & 200 & 1.158 & 5789 & 121 \\
\hline
\end{tabular}

Table 4. Experimental extinction coefficients for ionized pentabromophenol in saturated buffer at $\lambda=314.5$. The mean extinction coefficient, $\varepsilon$, is $5770 \pm 29 \mathrm{~cm}^{-1} \cdot \mathrm{M}^{-1}$. 


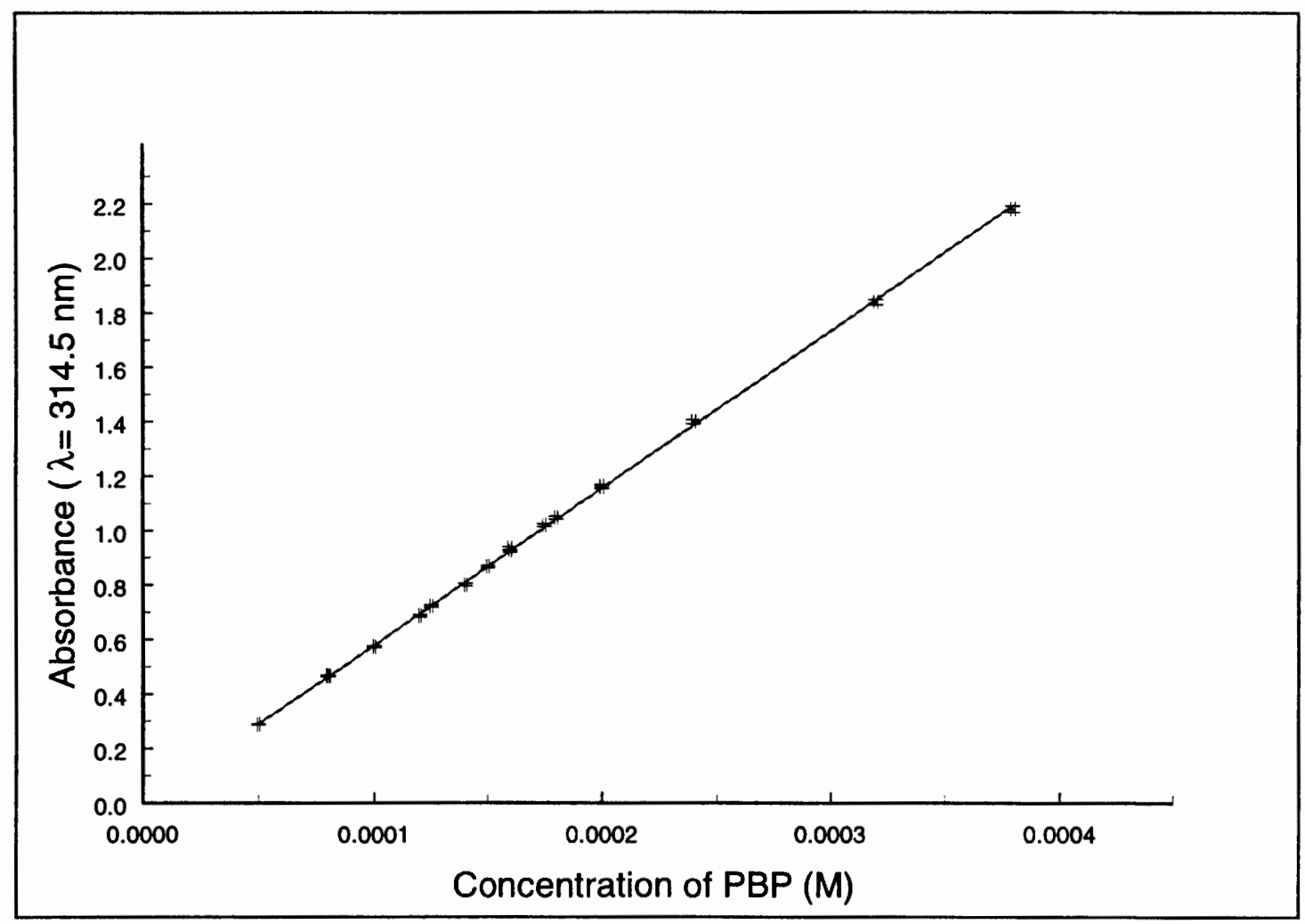

Figure 16. Absorbance versus concentration of ionized PBP at $\lambda=314.5 \mathrm{~nm}$. Sets of vertical and horizontal error bars enclose data points. The slope is the extinction coefficient. 


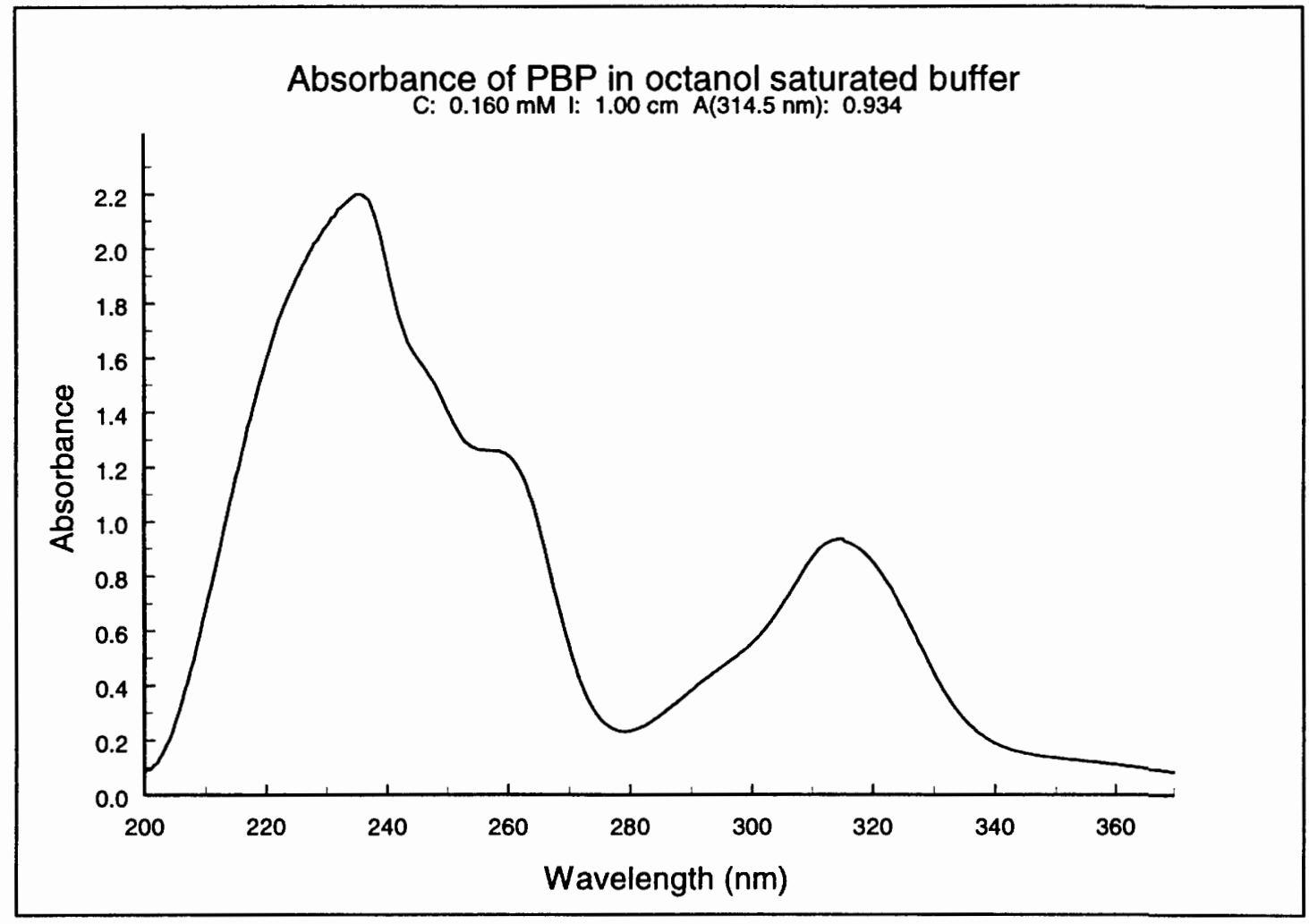

Figure 17. A typical absorbance spectrum of ionized PBP. The peak of interest is on the right, centered about $314.5 \mathrm{~nm}$. 


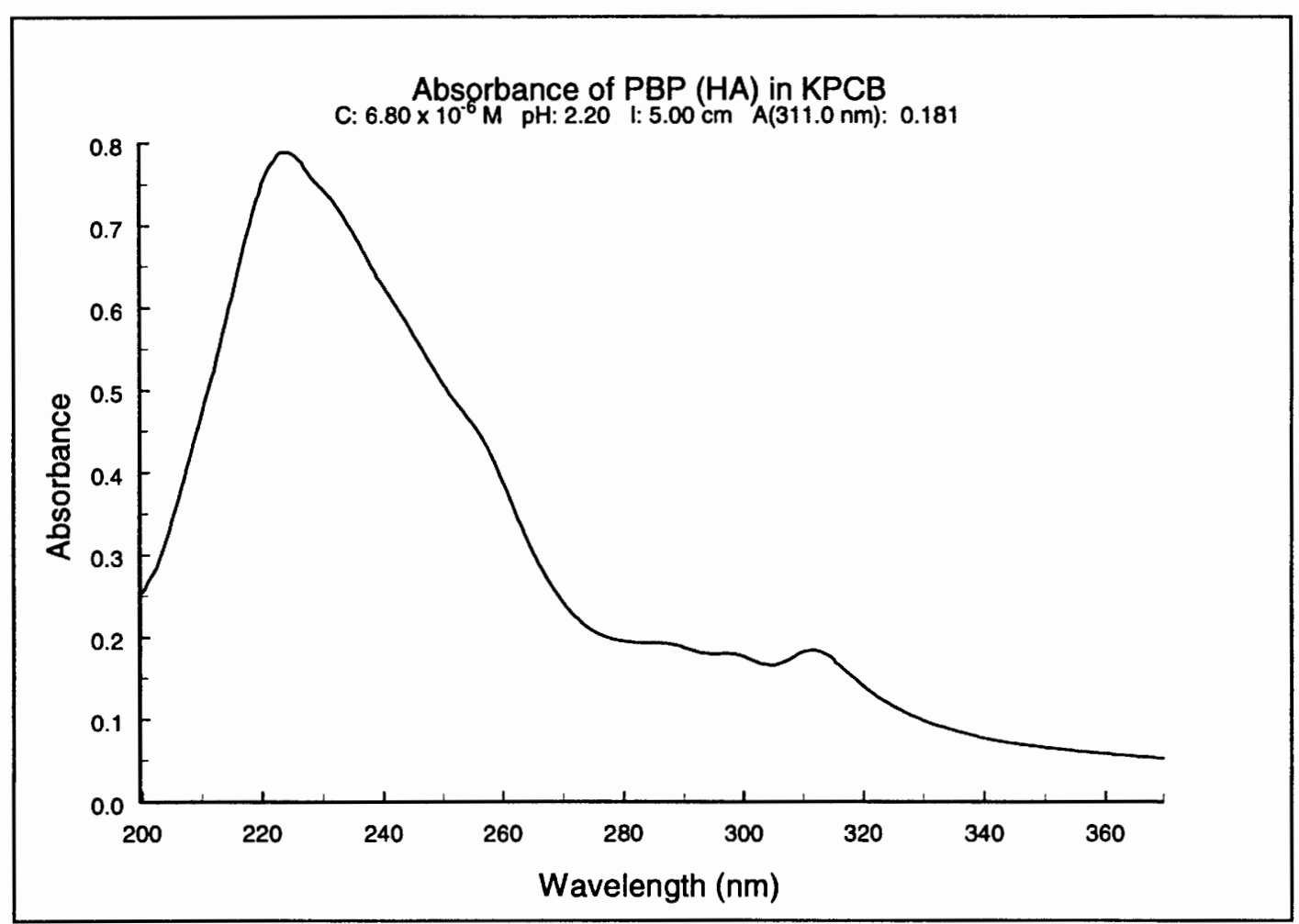

Figure 18. Absorbance of unionized PBP in unsaturated buffer. Due to the low solubility of the neutral species, the concentration is lower than the sample used in the previous spectrum (thus, the lower absorbances of the peaks). 


\section{Octanol Water Partitioning}

We have measured the distribution of 2,3,4,6-TeCP, PFP, PCP, and PBP as a function of $\mathrm{pH}$. Initial buffer-phase solutions have adjusted $\mathrm{pH}$ levels between 2.5 and 12.5. The initial octanol-phase phenol concentration is constant throughout all experimental trials of PFP and PBP. For TeCP and PCP, a second, higher concentration octanol-phase solution was used over the $\mathrm{pH}$ range 2.5 5. Buffer-phase concentrations were determined by UVVis spectroscopy and are adjusted for volume changes due to $\mathrm{KOH}$ titrations. ${ }^{7}$

Since we have no method for adjusting the ionization of phenols dissolved in the octanol-phase, spectra from this region display the over lapping contributions from both phenol species and have little value. For this reason, the octanol-phase concentrations are obtained from the mass-balance consideration:

$$
[\text { Phenol }]_{O}=[\text { Phenol }]_{\text {Initial }}-\frac{[\text { Phenol }]_{a q} V_{a q}}{V_{o}}
$$

Here we neglect adsorption to the glass wall of the cylinder, which is justified in view of the relatively large volumes used in these experiments. $V_{a q}$ is $25 \mathrm{ml}$ and $V_{O}$ is $5 \mathrm{ml}$. Tables 6-9 show the experimental results for each phenol.

From the acid to base region of the $\mathrm{pH}$ scale, the distribution ratio of each phenol varies over several orders of magnitude. Figures 19 to 22 show the logarithmic distribution of each phenol, as a function of $\mathrm{pH}$. The distribution model (equation 18; 34) describes our 2,3,4,6-TeCP and PFP experimental results very well (the solid line on Figures 19 and 20). The $\mathrm{pH}$ dependent distribution ratio $D$ is given by

\footnotetext{
7 The final phenol concentration is the concentration measured with the spectrophotometer multiplied by a correction factor given by $[\text { phenol }]_{\text {final }}=\left(V_{K O H}+V_{\text {initial }}\right) / V_{\text {initial }} \cdot[\text { phenol }]_{\text {measured }}$ (Tables 6-9). 


$$
D=\frac{g_{H A}+g_{A} 10^{p H-p K a}}{1+10^{p H-p K a}} .
$$

We can obtain the distribution coefficients, $g_{H A}$ for unionized and $g_{A}$ for ionized species, by taking the asymptotic limits of $\mathrm{D}$ as the $\mathrm{pH}$ approaches negative or positive infinity:

$$
\lim _{p H \rightarrow-\infty} D=g_{H A} \quad \lim _{p H \rightarrow \infty} D=g_{A} .
$$

From the best fit of our model to the experiment, we find for tetrachlorophenol:

$$
\begin{aligned}
& \mathrm{g}_{\mathrm{HA}}(2,3,4,6-\mathrm{TeCP})=18,900 \pm 700 \\
& \mathrm{~g}_{\mathrm{A}}(2,3,4,6-\mathrm{TeCP})=3.01 \pm 0.18 .
\end{aligned}
$$

For pentafluorophenol we obtained:

$$
\begin{aligned}
& \mathrm{g}_{\mathrm{HA}}(\mathrm{PFP})=610 \pm 17 \\
& \mathrm{~g}_{\mathrm{A}}(\mathrm{PFP})=0.140 \pm 0.007
\end{aligned}
$$

There is some difficulty fitting PCP data from the low $\mathrm{pH}$ range to the model. The experimental results indicate that:

$$
\begin{aligned}
& \mathrm{g}_{\mathrm{HA}}(\mathrm{PCP}) \cong 60,000 \pm 1,000 \\
& \mathrm{~g}_{\mathrm{A}}(\mathrm{PCP})=14.3 \pm 0.2
\end{aligned}
$$

Whereas the model suggests that $\mathrm{g}_{\mathrm{HA}}$ should be closer to 100,000 as shown in the expected distribution curve for PCP of Figure 21.

The results of the PBP experiments are inconsistent with the model. The experimental values for the distribution coefficients are estimated by the means of the low and high $\mathrm{pH}$ regions of the distribution curve:

$$
\mathrm{g}_{\mathrm{HA}}(\mathrm{PBP}) \cong 50,000 \pm 8,000
$$




$$
g_{A}(P B P)=59 \pm 3
$$

By excluding data near or below the $\mathrm{pKa}$ of PBP, we can fit the model to the remaining points, (Figure 22). Extrapolation of the curve to low pH suggests a $g_{A}$ value about $4 \times 10^{6}$.

A summary of the distribution parameters is given in Table 5. The more familiar partition coefficient, $\log \mathrm{K}_{\mathrm{OW}}$ is also included for discussion purposes.

Table 5. Distribution parameters of halogenated phenols.

\begin{tabular}{lllll}
\hline Halophenol & $g_{H A}$ & $g_{A}$ & $\log K_{\text {OW }}(\mathrm{HA})$ & $\log \mathrm{K}_{\mathrm{OW}}\left(\mathrm{A}^{-}\right)$ \\
\hline PFP & $610 \pm 17$ & $0.140 \pm 0.007$ & 2.79 & -0.854 \\
$2,3,4,6-\mathrm{TeCP}$ & $18,900 \pm 700$ & $3.01 \pm 0.18$ & 4.28 & 0.479 \\
PCP & $60,000 \pm 1,000$ & $14.3 \pm 0.2$ & 4.77 & 1.16 \\
PBP & $>50,000$ & $59 \pm 3$ & $>4.67$ & 1.77 \\
\hline
\end{tabular}




\section{Error Analysis of Distribution Experiments}

Uncertainties in distribution values from experiment are determined using the error propagation formula from the previous section (28). The uncertainty of $D$ from individual trials generally ranges from $2-3 \%$. However, when the concentration of one phase approaches zero (concentration $\leq 1 \mu \mathrm{M}$ ) large errors of up to $60 \%$ or $70 \%$ occur (high $\mathrm{pH}$ data for PFP and low $\mathrm{pH}$ data for the others). Vertical error bars in Figures 19 to 22 represent experimental uncertainties. Note that the logarithmic scale distorts the relative errors.

Uncertainty in the distribution coefficients, $g_{H A}$ and $g_{A}$, is the standard deviation confidence limits obtained from the Axum non-linear fit program. Where the model does not fit our data, the mean-deviation is used to approximate the experimentally determined $g_{H A}$ parameters of PCP and PBP. 


\begin{tabular}{|c|c|c|c|c|c|c|c|c|c|}
\hline $\begin{array}{l}\text { Stock } \\
(\mathrm{mM})\end{array}$ & $\begin{array}{c}\text { Initial } \\
\mathrm{pH} \\
\end{array}$ & $\begin{array}{l}\text { Final } \\
\mathrm{pH}\end{array}$ & $\begin{array}{c}\text { Titrated } \\
\mathrm{pH}\end{array}$ & $\begin{array}{c}\text { Cuvette } \\
\text { Length }(\mathrm{cm}) \\
\end{array}$ & A & $\frac{\left(V_{K O H}+V_{\text {initial }}\right)}{V_{\text {initial }}}$ & $\begin{array}{c}{[\mathrm{TeCP}]_{\mathrm{w}}} \\
(\mathrm{mM})\end{array}$ & $\begin{array}{c}{[\mathrm{TeCP}]_{\mathrm{o}}} \\
(\mathrm{mM})\end{array}$ & $\log D$ \\
\hline 19.97 & 8.15 & 7.65 & 10.10 & 1.00 & 0.828 & 1.017 & 0.1666 & 19.14 & 2.060 \\
\hline 19.97 & 9.13 & 7.93 & 10.01 & 1.00 & 1.450 & 1.018 & 0.2917 & 18.51 & 1.803 \\
\hline 19.97 & 10.32 & 8.25 & 10.15 & 0.100 & 0.328 & 1.008 & 0.6533 & 16.70 & 1.408 \\
\hline 19.97 & 10.88 & 8.71 & 10.11 & 0.100 & 0.587 & 1.025 & 1.190 & 14.02 & 1.071 \\
\hline 19.97 & 11.34 & 9.43 & 10.60 & 0.100 & 1.048 & 1.013 & 2.098 & 9.480 & 0.655 \\
\hline 19.97 & 11.70 & 10.43 & 10.43 & 0.100 & 1.245 & 1.000 & 2.461 & 7.663 & 0.493 \\
\hline 19.97 & 11.55 & 10.02 & 10.02 & 0.100 & 1.190 & 1.000 & 2.353 & 8.206 & 0.543 \\
\hline 19.97 & 11.82 & 11.39 & 11.39 & 0.100 & 1.257 & 1.000 & 2.485 & 7.544 & 0.482 \\
\hline 19.97 & 11.78 & 9.94 & 9.94 & 0.100 & 1.140 & 1.000 & 2.254 & 8.701 & 0.587 \\
\hline 19.97 & 6.61 & 6.68 & 10.15 & 1.00 & 0.091 & 1.000 & $17.99 \mu \mathrm{M}$ & 19.88 & 3.043 \\
\hline 19.97 & 5.56 & 5.62 & 9.99 & 5.00 & 0.073 & 1.012 & 2.921 & 19.96 & 3.835 \\
\hline 19.97 & 4.77 & 4.79 & 11.35 & 5.00 & 0.044 & 1.020 & 1.775 & 19.96 & 4.051 \\
\hline 19.97 & 5.94 & 5.95 & 10.37 & 5.00 & 0.110 & 1.010 & 4.393 & 19.95 & 3.657 \\
\hline 19.97 & 5.09 & 5.11 & 10.04 & 5.00 & 0.040 & 1.015 & 1.605 & 19.96 & 4.095 \\
\hline 66.24 & 4.75 & 4.77 & 10.18 & 5.00 & 0.090 & 1.066 & 3.812 & 66.22 & 4.240 \\
\hline 66.24 & 3.60 & 3.58 & 10.13 & 5.00 & 0.083 & 1.081 & 3.526 & 66.22 & 4.274 \\
\hline
\end{tabular}

Table 6. Distribution data for 2,3,4,6-tetrachlorophenol. 


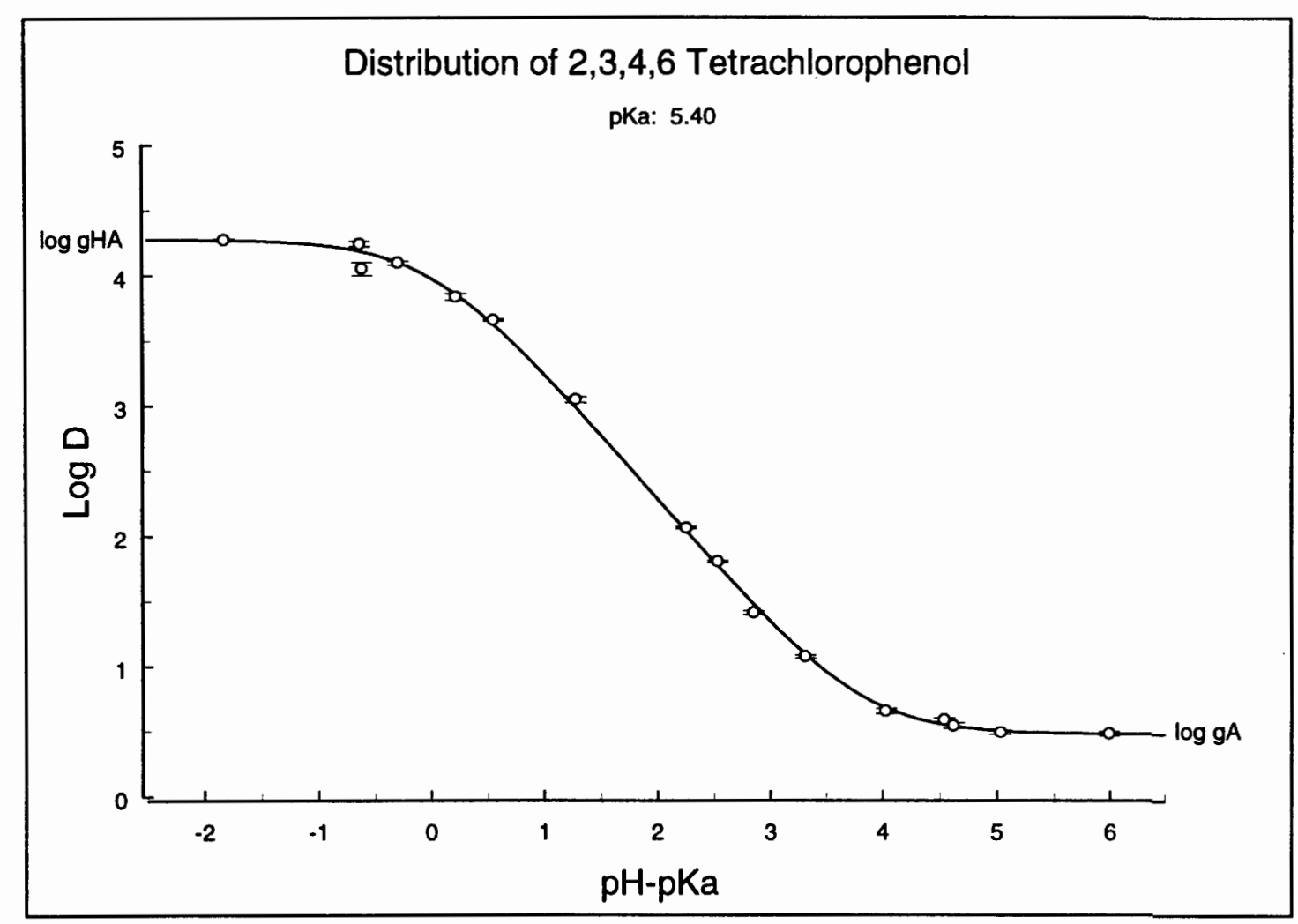

Figure 19. Distribution of 2,3,4,6, TeCP. The pKa of 2,3,4,6-TeCP is 5.40 \{Schellenberg, et al. 1984\}. 


\begin{tabular}{|c|c|c|c|c|c|c|c|c|c|}
\hline $\begin{array}{l}\text { Stock } \\
(\mathrm{mM})\end{array}$ & $\begin{array}{c}\text { Initial } \\
\mathrm{pH}\end{array}$ & $\begin{array}{c}\text { Final } \\
\mathrm{pH}\end{array}$ & $\begin{array}{c}\text { Titrated } \\
\mathrm{pH}\end{array}$ & $\begin{array}{l}\text { Path Length } \\
(\mathrm{cm})\end{array}$ & $\mathrm{A}$ & $\frac{\left(V_{K O H}+V_{\text {initial }}\right)}{V_{\text {initial }}}$ & $\begin{array}{c}{[\mathrm{PFP}]_{\text {buffer }}} \\
(\mathrm{mM})\end{array}$ & $\begin{array}{c}{[\mathrm{PFP}]_{\text {octanol }}} \\
(\mathrm{mM})\end{array}$ & $\log D$ \\
\hline 21.00 & 8.27 & 7.12 & 10.89 & 1.00 & 1.270 & 1.033 & 1.020 & 15.90 & 1.1928 \\
\hline 21.00 & 10.26 & 7.37 & 10.20 & 1.00 & 1.816 & 1.021 & 1.442 & 13.79 & 0.9804 \\
\hline 21.00 & 11.06 & 7.71 & 10.54 & 0.100 & 0.281 & 1.025 & 2.240 & 9.802 & 0.6411 \\
\hline 21.00 & 11.86 & 11.05 & 11.05 & 0.100 & 0.523 & 1.000 & 4.067 & 0.666 & -0.7860 \\
\hline 21.00 & 11.34 & 8.17 & 10.65 & 0.100 & 0.401 & 1.024 & 3.196 & 5.019 & 0.1960 \\
\hline 21.00 & 11.60 & 9.69 & 10.26 & 0.100 & 0.519 & 1.008 & 4.068 & 0.662 & -0.7885 \\
\hline 21.00 & 11.48 & 8.43 & 10.05 & 0.100 & 0.454 & 1.010 & 3.569 & 3.156 & -0.0534 \\
\hline 21.00 & 12.14 & 11.80 & 11.80 & 0.100 & 0.525 & 1.000 & 4.084 & 0.580 & -0.8476 \\
\hline 21.00 & 3.05 & 3.13 & 11.59 & 5.00 & 0.208 & 1.179 & 0.0381 & 20.81 & 2.7371 \\
\hline 21.00 & 11.55 & 10.94 & 10.94 & 0.100 & 0.526 & 1.000 & 4.092 & 0.541 & -0.8785 \\
\hline 21.00 & 3.64 & 3.70 & 10.83 & 5.00 & 0.179 & 1.033 & 0.0288 & 20.86 & 2.8605 \\
\hline 21.00 & 5.80 & 5.87 & 11.08 & 1.00 & 0.131 & 1.071 & 0.1088 & 20.46 & 2.2743 \\
\hline 21.00 & 2.81 & 2.80 & 10.90 & 5.00 & 0.198 & 1.140 & 0.0351 & 20.82 & 2.7732 \\
\hline 21.00 & 4.51 & 4.48 & 10.83 & 5.00 & 0.219 & 1.186 & 0.0403 & 20.80 & 2.7123 \\
\hline 21.00 & 4.98 & 5.00 & 10.44 & 5.00 & 0.256 & 1.069 & 0.0425 & 20.79 & 2.6890 \\
\hline 21.00 & 6.43 & 6.36 & 10.64 & 5.00 & 1.700 & 1.046 & 0.2766 & 19.62 & 1.8508 \\
\hline
\end{tabular}

Table 7. Distribution data for PFP. 


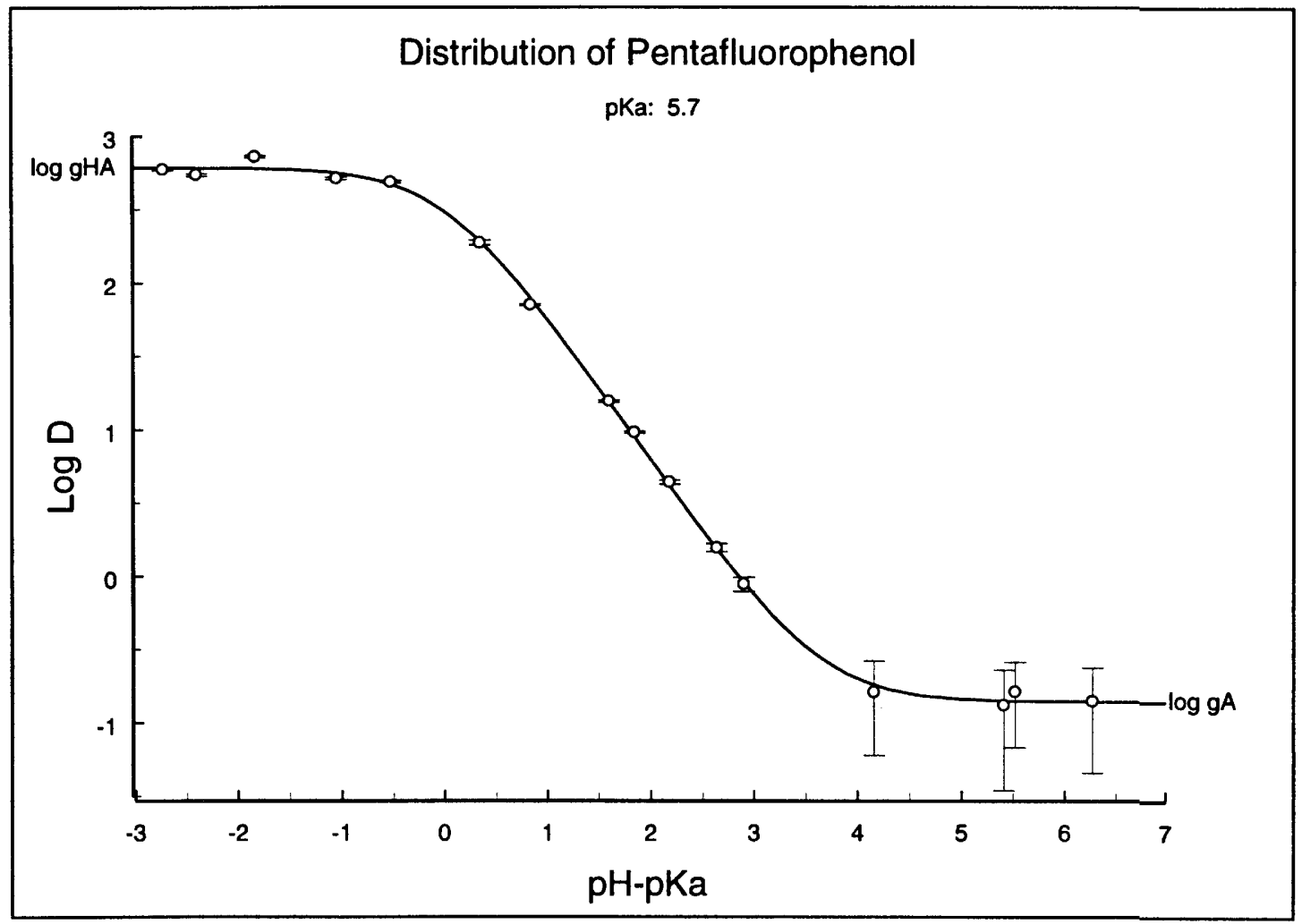

Figure 20. Distribution of pentafluorophenol. The pKa of PFP is 5.7 \{Arnold, et al. 1988\}. 


\begin{tabular}{|c|c|c|c|c|c|c|c|c|c|}
\hline $\begin{array}{l}\text { Stock } \\
(\mathrm{mM}) \\
\end{array}$ & $\begin{array}{c}\text { Initial } \\
\mathrm{pH}\end{array}$ & $\begin{array}{c}\text { Final } \\
\mathrm{pH}\end{array}$ & $\begin{array}{c}\text { Titrated } \\
\mathrm{pH}\end{array}$ & $\begin{array}{c}\text { Path Length } \\
(\mathrm{cm})\end{array}$ & A & $\frac{\left(V_{K O H}+V_{\text {initial }}\right)}{V_{\text {initial }}}$ & $\begin{array}{c}{[\mathrm{PCP}]_{\text {buffer }}} \\
(\mathrm{mM})\end{array}$ & $\begin{array}{c}{[\mathrm{PCP}]_{\text {octanol }}} \\
(\mathrm{mM})\end{array}$ & $\log D$ \\
\hline 20.00 & 9.50 & 7.89 & 10.19 & 1.00 & 0.819 & 1.010 & 0.1638 & 19.18 & 2.068 \\
\hline 20.00 & 8.38 & 7.67 & 10.20 & 1.00 & 0.528 & 1.0125 & 0.1058 & 19.47 & 2.265 \\
\hline 20.00 & 8.34 & 7.66 & 10.07 & 1.00 & 0.495 & 1.015 & 0.0996 & 19.50 & 2.292 \\
\hline 20.00 & 10.38 & 8.12 & 10.41 & 1.00 & 1.162 & 1.0125 & 0.2329 & 18.83 & 1.908 \\
\hline 20.00 & 11.16 & 8.54 & 10.20 & 0.100 & 0.289 & 1.0085 & 0.5779 & 17.39 & 1.471 \\
\hline 20.00 & 11.98 & 10.24 & 10.24 & 0.100 & 0.534 & 1.000 & 1.057 & 14.71 & 1.143 \\
\hline 20.00 & 5.88 & 5.96 & 10.61 & 5.00 & 0.097 & 1.0405 & $3.989 \mu \mathrm{M}$ & 19.98 & 3.700 \\
\hline 20.00 & 11.50 & 8.69 & 10.74 & 0.100 & 0.299 & 1.0135 & $0.6001 \mathrm{mM}$ & 17.00 & 1.452 \\
\hline 20.00 & 11.80 & 9.25 & 10.14 & 0.100 & 0.436 & 1.0065 & 0.8690 & 15.66 & 1.256 \\
\hline 20.00 & 12.20 & 11.31 & 11.31 & 0.100 & 0.527 & 1.000 & 1.045 & 14.77 & 1.151 \\
\hline 20.00 & 6.51 & 6.52 & 11.07 & 5.00 & 0.246 & 1.035 & $1.007 \mu \mathrm{M}$ & 19.94 & 3.297 \\
\hline 100.0 & 2.96 & 2.95 & 11.22 & 5.00 & 0.040 & 1.095 & 1.717 & 99.99 & 4.765 \\
\hline 100.0 & 3.94 & 3.98 & 10.13 & 5.00 & 0.045 & 1.065 & 1.915 & 99.99 & 4.718 \\
\hline 100.0 & 4.77 & 4.79 & 10.59 & 5.00 & 0.057 & 1.0675 & 2.393 & 99.98 & 4.621 \\
\hline 100.0 & 3.46 & 3.48 & 10.94 & 5.00 & 0.038 & 1.098 & 1.657 & 99.99 & 4.781 \\
\hline
\end{tabular}

Table 8. Distribution data for PCP. 


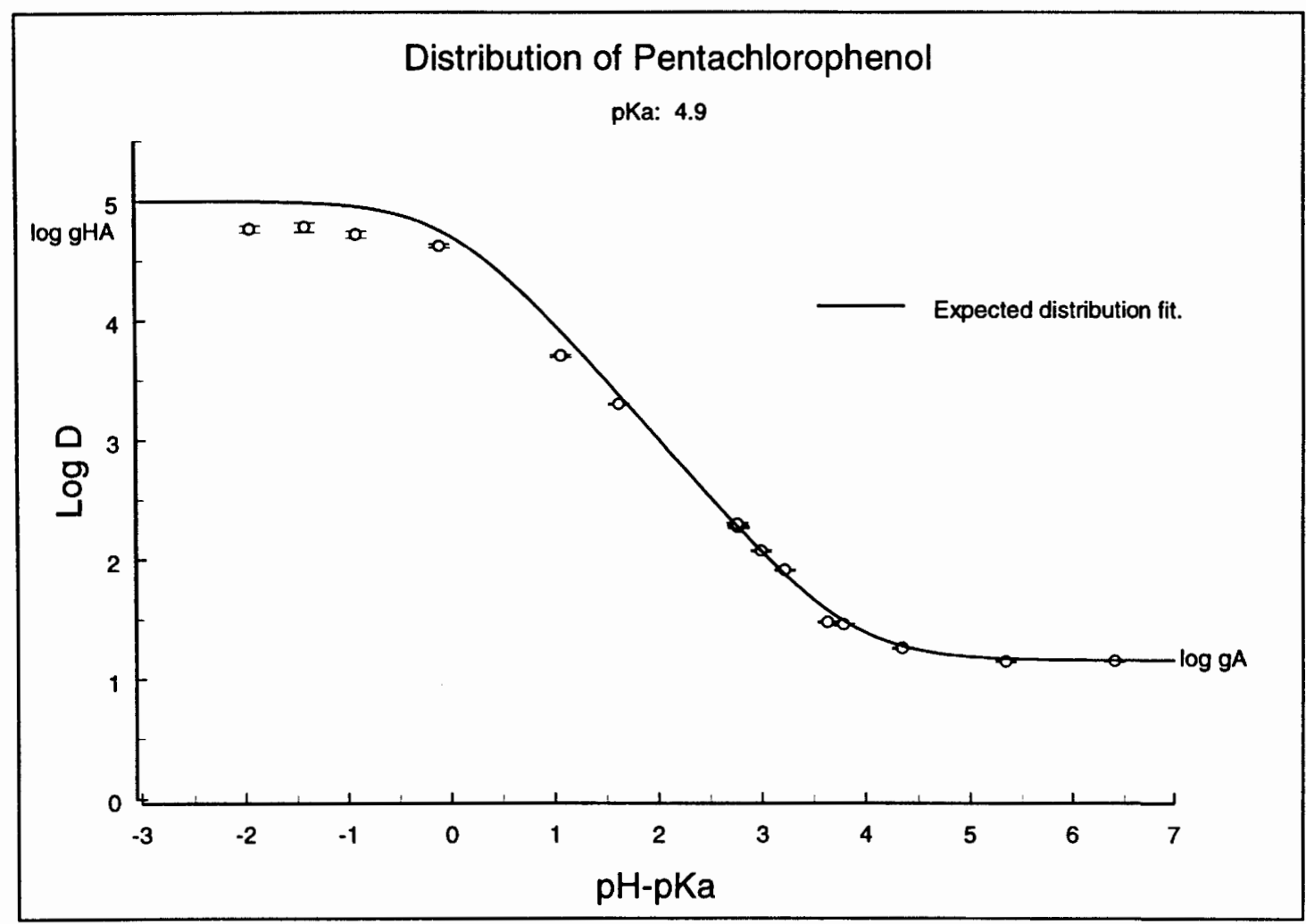

Figure 21. Data and expected distribution of PCP. The pKa of PCP is 4.9\{Westall, et al. 1985\}. 


\begin{tabular}{|c|c|c|c|c|c|c|c|c|c|}
\hline $\begin{array}{l}\text { Stock } \\
(\mathrm{mM})\end{array}$ & $\begin{array}{c}\text { Initial } \\
\mathrm{pH}\end{array}$ & $\begin{array}{c}\text { Final } \\
\mathrm{pH}\end{array}$ & $\begin{array}{c}\text { Titrated } \\
\text { pH }\end{array}$ & $\begin{array}{l}\text { Path Length } \\
\text { (cm) }\end{array}$ & A & $\frac{\left(V_{K O H}+V_{\text {initial }}\right)}{V_{\text {initial }}}$ & $\begin{array}{c}{[\mathrm{PBP}]_{\text {buffer }}} \\
(\mu \mathrm{M})\end{array}$ & $\begin{array}{l}{[\text { PBP }]_{\text {octanol }}} \\
(\mathrm{mM})\end{array}$ & $\log D$ \\
\hline 90.00 & 8.33 & 7.24 & 10.88 & 1.000 & 0.377 & 1.026 & 66.95 & 89.67 & 3.127 \\
\hline 90.00 & 11.02 & 7.60 & 10.65 & 1.000 & 0.794 & 1.020 & 140.3 & 89.30 & 2.804 \\
\hline 90.00 & 11.96 & 8.74 & 10.13 & 0.100 & 0.520 & 1.010 & 908.8 & 85.46 & 1.973 \\
\hline 90.00 & 12.48 & 11.46 & 11.46 & 0.100 & 0.815 & 1.000 & 1412 & 82.94 & 1.769 \\
\hline 90.00 & 12.11 & 8.60 & 10.00 & 0.100 & 0.427 & 1.016 & 750.9 & 86.25 & 2.060 \\
\hline 90.00 & 12.30 & 8.83 & 10.65 & 0.100 & 0.535 & 1.017 & 942.3 & 85.29 & 1.957 \\
\hline 90.00 & 5.60 & 5.54 & 11.48 & 5.000 & 0.104 & 1.089 & 3.915 & 89.98 & 4.361 \\
\hline 90.00 & 6.79 & 6.62 & 10.61 & 1.000 & 0.102 & 1.038 & 18.32 & 89.91 & 3.691 \\
\hline 90.00 & 4.75 & 4.69 & 9.69 & 5.000 & 0.051 & 1.074 & 1.906 & 89.99 & 4.674 \\
\hline 90.00 & 6.12 & 6.06 & 9.03 & 5.000 & 0.208 & 1.039 & 7.492 & 89.96 & 4.079 \\
\hline 90.00 & 2.62 & 2.57 & 9.05 & 5.000 & 0.049 & 1.138 & 1.940 & 89.99 & 4.666 \\
\hline 90.00 & 3.49 & 3.41 & 10.73 & 5.000 & 0.053 & 1.105 & 2.030 & 89.99 & 4.647 \\
\hline 90.00 & 3.03 & 3.02 & 9.72 & 5.000 & 0.050 & 1.080 & 1.871 & 89.99 & 4.682 \\
\hline 90.00 & 12.47 & 8.98 & 8.98 & 0.100 & 0.656 & 1.000 & 1136 & 84.32 & 1.871 \\
\hline 90.00 & 12.34 & 11.29 & 11.29 & 0.100 & 0.839 & 1.000 & 1454 & 82.73 & 1.755 \\
\hline 90.00 & 12.30 & 11.93 & 11.93 & 0.100 & 0.770 & 1.000 & 1334 & 83.33 & 1.796 \\
\hline 90.00 & 3.05 & 3.05 & 11.87 & 5.000 & 0.032 & 1.199 & 1.330 & 89.99 & 4.830 \\
\hline
\end{tabular}

Table 9. Distribution data for PBP. 


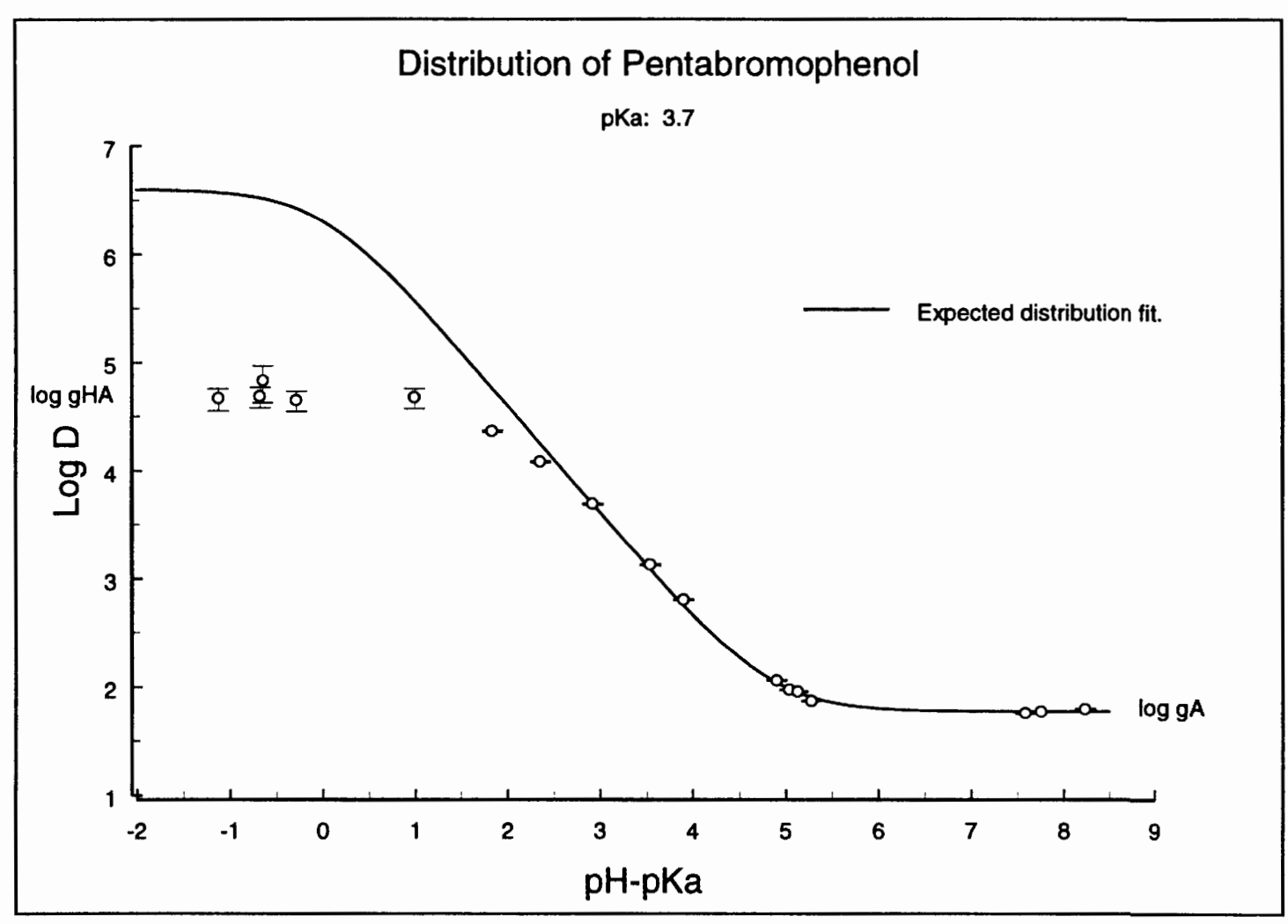

Figure 22. Data and expected distribution of PBP. The pKa of PBP is 3.7 \{Arnold 1988\}. 


\section{Discussion}

Except for unionized pentabromophenol, experimental results agree with our distribution model. Ionized halophenols distribute quite differently than their neutral counterparts.

For discussion, two partitioning models based on the physical properties of molecules are presented. The difference between octanol-water and lipid membranewater partitioning behavior within the framework of a lipid membrane-water model is also discussed. Remarks on the limits of the "shake flask" method and a summary of conclusions follow.

\section{Molar volume model of two-phase solute partitioning}

The partition coefficient for a class of physically similar molecules is linearly correlated with the molar volume of the solute (Gobas 1988). The partition coefficient is defined as

$$
K_{O W}=\frac{[H A]_{O}}{[H A]_{W}}=\frac{V_{W} \gamma_{W}}{V_{o} \gamma_{o}}
$$

$V_{W}$ and $V_{O}$ are the molar volumes of the octanol and water phases (molecular weight divided by density) and $\gamma_{\mathrm{W}}$ and $\gamma_{\mathrm{O}}$ are the activity coefficients. The activity coefficients are related to the Gibb's free energy of solution $\Delta G_{S}$ by

$$
\ln \gamma=\Delta G_{S} / R T .
$$

Where $\mathrm{R}$ is the molar gas constant and $T$ is the temperature in Kelvin. The free 
energy of solution can be expressed as the sum of the free energy of solvation and the free energy of vaporization,

$$
\Delta G_{S}=\Delta G_{S V}+\Delta G_{V}
$$

The free energy of solvation may be further expanded as the sum of the energy required to create a cavity in the medium (or collapse the cavity) $\Delta G_{c}$, and the free energy required for a solute molecule to occupy (or vacate) the cavity $\Delta G_{i}$ :

$$
\Delta G_{S}=\left(\Delta G_{c}+\Delta G_{i}\right)+\Delta G_{V} .
$$

Combining equations 37,38 , and 40 gives an expression for the partition coefficient in terms of the molar volumes of the solvents and the cavity and occupation free energies associated with the solute molecule:

$$
K_{O W}=\frac{V_{W}}{V_{O}}\left\{e^{\left(\Delta G_{c, W}-\Delta G_{c, o}\right)+\left(\Delta G_{i, W}-\Delta G_{i, o}\right)}\right\}^{1 / R T} .
$$

To simplify the equation, Gobas, et al., makes the following approximations. For a class of geometrically similar molecules, they assume that the free energies of cavity formation are linearly proportional to the molar volume of the solute such that:

$$
\Delta G_{c, W}-\Delta G_{c, o}=(W-O) V_{s}
$$

Where $W$ and $O$ are cavity constants of the water and octanol phases. Furthermore, for chemically similar molecules, they assume that the occupation or introduction free energies are constants. The molar volumes of the phases are also constants so we arrive at a relation for $K_{O W}$ with one dependent variable, $V_{S}$ : 


$$
K_{O W}=k e^{(W-O) V_{S} / R T} .
$$

Taking decadic logarithms of both sides and introducing $C$, the cavity constant, and $I$, the solute introduction constant, we arrive at a linear relationship between Log $K_{O W}$ and $V_{s}$ :

$$
\log K_{\text {OW }}=C V_{S}+I \text {. }
$$

Molar volumes and partition coefficients for halophenols are given in Table 10. For comparison, physically similar halobenzenes are also included. Partition coefficients of the chlorobenzenes were measured by deBruijn (1988) using the more accurate "slow-stirring" method. Molar volumes were calculated using Molecular Modeling Pro (Windowchem Software). Linear plots of Log $\mathrm{K}_{\mathrm{OW}}$ versus molar volume for halobenzenes and halophenols are shown in Figure 23. Due to the uncertainty of $\log \mathrm{K}_{\mathrm{OW}}$ for PBP, it is excluded from the linear regression calculations.

Plots of $\log K_{O W}$ versus $V_{S}$ for ionized and unionized halophenols are shown in Figure 24. In this case, the molar volume of ionized halophenols is assumed to be the same as $V_{S}$ for the unionized species.

The cavity constants and introduction constants for the selected molecules are given in Table 11. Even with this small sample size, we can make some conclusions from these results.

The cavity formation parameters (the slope $C$ ) are about the same for bromobenzenes, chlorobenzenes, and halophenols $\left(0.041,0.044\right.$, and $\left.0.050 \mathrm{~mol} / \mathrm{cm}^{3}\right)$. This is expected since these aromatic molecules have about the same shape. $C$ is 
also the same for ionized and neutral halophenols $\left(0.049\right.$ versus $\left.0.050 \mathrm{~mol} / \mathrm{cm}^{3}\right)$. This suggests that molar volumes are not dependent on charge.

The solvent-solute interactions are more important then assumed in the development of this model. The molecular introduction constant (the intercept $I$ ) is only constant for a narrow class of molecules. Even the geometrically identical bromobenzenes behave quite differently then the chlorobenzenes $(I=-1.47$ versus -0.399). The single fluorine solute, pentafluorophenol, fits well with the chlorophenols, however. By comparison with the other bromine-containing compounds, we conclude that the partition coefficient of unionized PBP should have a much higher value then measured. Using $C$ and $I$ from the plot of the other phenols, the model predicts partition coefficients of 5.42 and 1.81 for neutral and ionized pentabromophenols respectively. The experimental Log $K_{\text {OW }}$ for ionized PBP is 1.77 and is in surprising agreement with the predicted value.

Isomers have the same calculated molar volumes but rarely have identical partition coefficients. Partition coefficients predicted from this model for isomers are identical and thus, only approximately correct.

Finally, since $I$ is a simple experimentally determined constant, we lose the ability to rationally predict the solute-solvent interaction mechanisms of other compounds. 
Table 10. Molar volumes of halophenols, halophenolates, and selected halobenzenes.

\begin{tabular}{llll}
\hline Solute & $\begin{array}{l}\mathrm{V}_{\mathrm{S}}(\mathrm{MMP}) \\
\mathrm{cm}^{3} / \mathrm{mol}\end{array}$ & $\log \mathrm{K}_{\mathrm{OW}}$ & $\begin{array}{l}\text { Log } \mathrm{K}_{\mathrm{OW}} \\
\left(\mathrm{A}^{-}\right)\end{array}$ \\
\hline Halobenzenes & & & \\
1,3,5 Trichlorobenzene & 126.6 & $4.139^{\mathrm{a}}$ & - \\
1,2,4 Trichlorobenzene & 126.6 & $4.050^{\mathrm{a}}$ & - \\
1,2,3,5 Tetrachlorobenzene & 139.0 & $4.658^{\mathrm{a}}$ & - \\
1,2,4,5 Tetrachlorobenzene & 139.0 & $4.604^{\mathrm{a}}$ & - \\
Pentachlorobenzene & 151.4 & $5.183^{\mathrm{a}}$ & - \\
Hexachlorobenzene & 163.8 & $5.731^{\mathrm{a}}$ & - \\
1,4 Dibromobenzene & 118.7 & $4.30^{\mathrm{b}}$ & - \\
1,3,5 Tribromobenzene & 133.3 & $5.26^{\mathrm{b}}$ & - \\
Hexabromobenzene & 177.2 & $6.80^{\mathrm{b}}$ & - \\
Halophenols & & & \\
2,4,6-Trichlorophenol & 124.8 & $3.65^{\mathrm{c}}$ & $0.15^{\mathrm{c}}$ \\
2,3,4,5-Tetrachlorophenol & 137.3 & $4.52^{\mathrm{d}}$ & $0.87^{\mathrm{d}}$ \\
2,3,4,6-Tetrachlorophenol & 137.3 & 4.28 & 0.048 \\
Pentafluorophenol & 108.6 & 2.79 & -0.085 \\
Pentachlorophenol & 149.7 & 4.77 & 1.16 \\
Pentabromophenol & 160.8 & $4.67^{*}$ & 1.77 \\
\hline
\end{tabular}
a. deBruijn (1989)
b. Gobas (1988)
c. Sieder (1995)
d. Schmidt (1995)
* Not reliable.

Table 11. Cavity and introduction constants.

\begin{tabular}{llll}
\hline Solute & $\mathrm{C}\left(\mathrm{mol} / \mathrm{cm}^{3}\right)$ & $\mathrm{I}$ & $\mathrm{r}^{2}$ \\
\hline Chlorobenzenes & $.044 \pm 0.001$ & $-1.47 \pm 0.16$ & 0.997 \\
Bromobenzenes & $.041 \pm 0.006$ & $-0.40 \pm 0.89$ & 0.957 \\
Halophenols & $.050 \pm 0.005$ & $-2.62 \pm 0.68$ & 0.959 \\
Halophenolates (A) & $.049 \pm 0.006$ & $-6.07 \pm 0.81$ & 0.941 \\
\hline
\end{tabular}




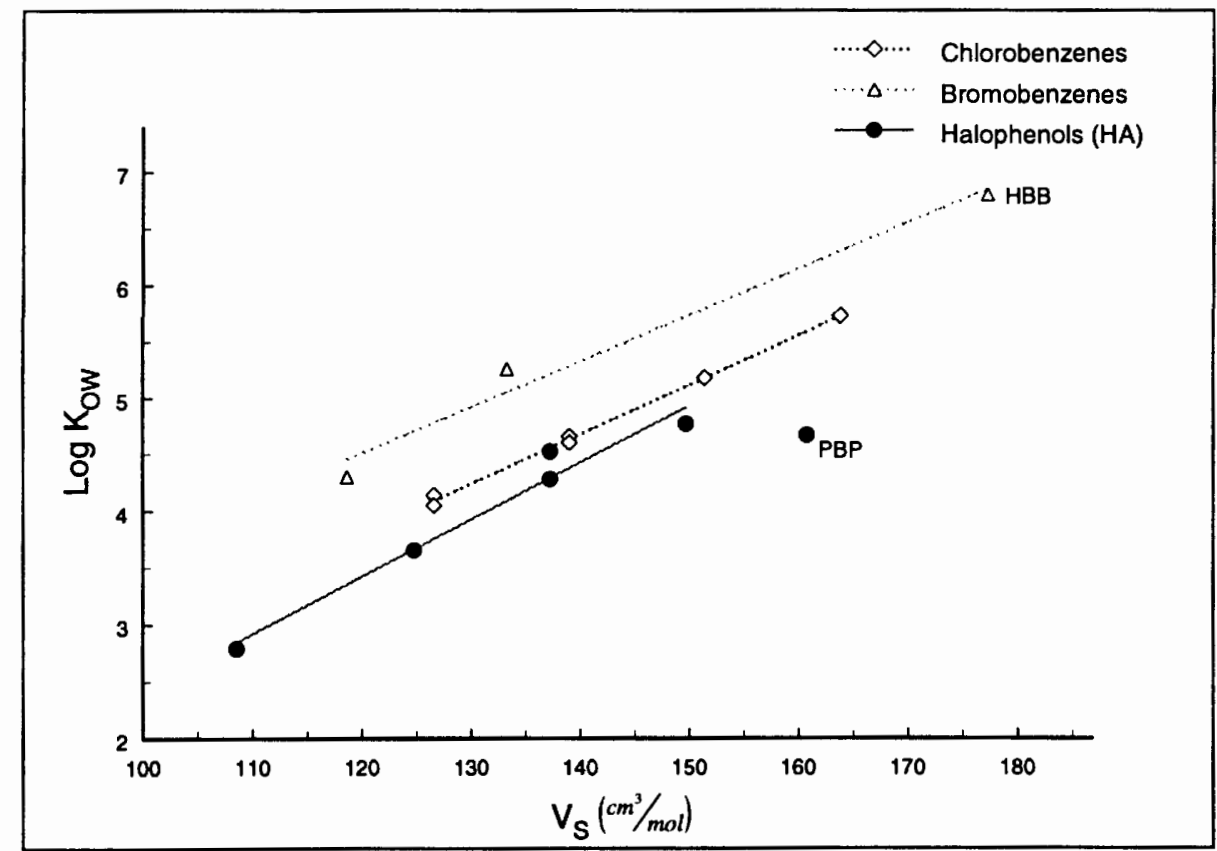

Figure 23. Correlation between $\log \mathrm{K}_{\mathrm{OW}}$ and solute molar volume $\mathrm{V}_{\mathrm{S}}$. 


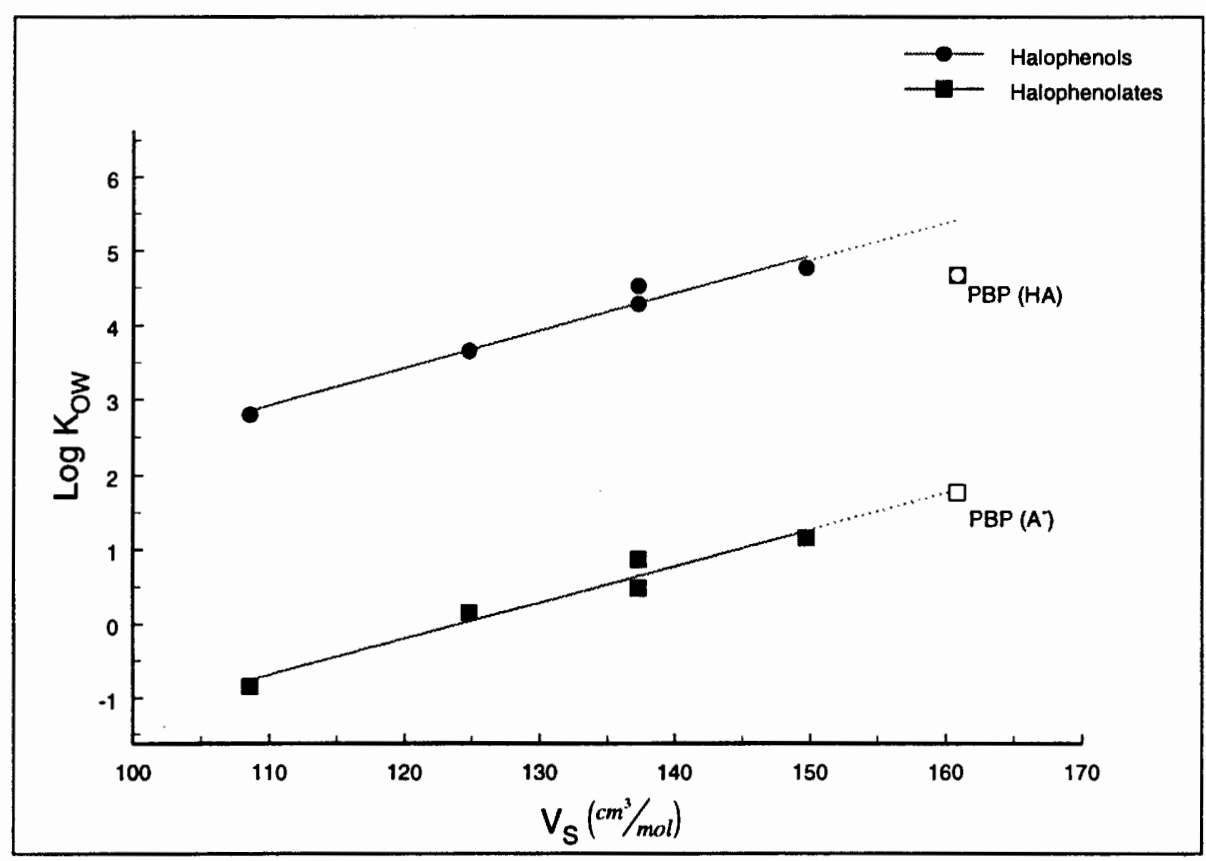

Figure 24. $\log K_{O W}$ versus $V_{S}$ for ionized and unionized halogenated phenols. The dotted lines represent extrapolations to data points for pentabromophenol. 


\section{Molecular surface area model of two-phase partitioning}

Partitioning of a solute may also be described in terms of molar fractions and correlated with the molecular surface area, MSA (Smejtek, et al. 1996). The definition of the molar fraction partition coefficient is:

$$
K_{p, O W}=\frac{n_{O}}{n_{a q}} .
$$

Where $n_{O}$ and $n_{a q}$ are the octanol and aqueous phase molar fractions given by

$$
n_{O}=\frac{[H A]_{O}}{[H A]_{O}+[\text { Octanol }]_{O}}
$$

and

$$
n_{a q}=\frac{[H A]_{a q}}{[H A]_{a q}+\left[H_{2} O\right]_{a q}} .
$$

The advantage of the molar fraction approach is that the partition coefficient can be described in terms of a change in the free energy of transfer $\Delta G_{O W}$ by the relation

$$
K_{p, O W}=e^{-\Delta G_{o w} / R T}
$$

The molar partition coefficient $K_{p, O w}$ and the partition coefficient $K_{O w}$ are related by

$$
K_{p, \text { OW }}=K_{\text {oW }} \frac{\left[\mathrm{H}_{2} \mathrm{O}\right]_{a q}}{[\text { Octanol }]_{O}}
$$

The combination of equations 48 and 49 is a relation between the partition 
coefficient and the Gibb's free energy of transfer:

$$
K_{\text {ow }}=\frac{[\text { Octanol }]_{O}}{\left[\mathrm{H}_{2} \mathrm{O}\right]_{a q}} e^{-\Delta G_{o w} / R T}
$$

The solubility of octanol in water is about $4-5 \times 10^{2}$ solubility of water in octanol so we may assume a pure water aqueous phase (Smejtek et al. 1996). The molar concentration of water in the aqueous phase is thus $55.41 \mathrm{M}^{8}$ at $20^{\circ} \mathrm{C}$. The concentration of octanol in the octanol phase is $6.15 \mathrm{M}$ (Bowman \& Sans 1983) and reflects the contribution of dissolved water to the total volume. We can now write $\Delta G_{\text {ow }}$ from equation 50 as

$$
\Delta G_{O W}=-R T\left(\ln 9.01+\ln K_{O W}\right) .
$$

Using decadic logarithms and $\mathrm{RT}=0.582 \mathrm{kcal} / \mathrm{mol}($ at $293 \mathrm{~K}$ ) we arrive at a relationship between the Gibb's free energy of transfer and the partition coefficient:

$$
\Delta G_{O W}=-\left(1.28+1.34 \log K_{O W}\right) k c a l / m o l
$$

Now we assume that the free energy of transfer can be separated into an electrostatic contribution $\Delta G_{e l}$ and a non-electrostatic contribution $\Delta G_{n o n-e l}$ as

$$
\Delta G_{o w}=\Delta G_{n o n-e l}+\Delta G_{e l} .
$$

Where $\Delta G_{e l}$ is dependent upon ion charge contributions and other factors not dominated by size characteristics.

8 The molar concentration of water in the aqueous phase was calculated using a water density of 998.23 gm/l (CRC handbook 1970). 
The non-electrostatic portion of the free energy of transfer is expected to be related to surface tensions and thus proportional to the "hydrophobic" nature of the solute and the molecular surface area MSA exposed to solution molecules. To demonstrate this correlation between $\Delta G_{\text {non-el }}$ and MSA we first introduce the hydrophobicity parameter $\alpha$ and assume the linear relationship

$$
\Delta G_{n o n-e l}=\alpha M S A
$$

The partition coefficient may now be written in terms of the molecular surface area, the electrostatic change in free energy, and the hydrophobicity parameter as the linear function

$$
\log K_{\text {OW }}=-\left(0.746 \alpha M S A+0.746 \Delta G_{e l}\right) \mathrm{mol} / \mathrm{kcal}-1.28 \text {. }
$$

To validate equation 55 , plots of $\log \mathrm{K}_{\mathrm{Ow}}$ versus MSA are shown for halobenzenes and halophenols (Figure 25) and halophenols and halophenolates (Figure 26). Partition coefficients and molecular surface areas $^{9}$ (generated by MMP) of halophenols, halophenolates, and halobenzenes are given in Table 12.

The hydrophobicity parameters and electrostatic free energies (Table 13) are obtained from linear regression analysis of the plots and equation 55 by the relations:

$$
\alpha=-1.34 \text { Slope } \cdot \mathrm{mol} / \mathrm{kcal}
$$

and

$$
\Delta G_{e l}=-1.34(\text { Intercept }+1.28) \mathrm{kcal} / \mathrm{mol} \text {. }
$$

\footnotetext{
9 Molecular surface area is the total surface generated by connected spheres representing atoms with
} van der Waals radii. 
The hydrophobicity parameters for halophenols is somewhat lower then for chlorobenzenes as predicted by the Gobas model $(-4.95 \pm 0.36$ versus $-3.95 \pm 0.11$ $\mathrm{kcal} \cdot \mathrm{mol}^{-1} \cdot \mathrm{nm}^{-2}$ ). Perhaps even more interesting is that the hydrophobicity parameters for ionized and unionized phenols are the same $(4.95 \pm 0.36$ versus $4.80 \pm 0.74$ $\mathrm{kcal} \cdot \mathrm{mol}^{-1} \cdot \mathrm{nm}^{-2}$ ). This fact supports our assumption that $\Delta G_{n o n-e l}$ and $\Delta G_{e l}$ are separate contributors to the free energy of transfer $\Delta G_{o w}$.

The electrostatic free energy of transfer for halophenols is positive $(1.84$ $\mathrm{kcal} / \mathrm{mol})$ and negative for chloro- and bromobenzenes $(-1.05$ and $-2.83 \mathrm{kcal} / \mathrm{mol}$ respectively). This probably reflects the hydrophilic polar nature of the phenol $\mathrm{OH}$ group.

Unlike the cavity and interaction coefficients of the Gobas model, the effects of ionic charges are readily included in the electrostatic term $\Delta G_{e l}$. The ionic contribution to the free energy of transfer for ionized phenols is an increase of +4.6 $\mathrm{kcal} / \mathrm{mol}$. This reflects significantly lower partition coefficients for the $\mathrm{A}^{-}$species of each phenol. The ionic contribution to $\Delta G_{O w}$ for pentafluorophenol is comparable to that of surface tension effects and leads to preferential partitioning in the polar aqueous phase.

The model based on molecular surface areas also predicts a higher partition coefficient for pentabromophenol. If PBP behaves similarly to the other halophenols, we should expect $\log K_{\text {OW }}$ of PBP to be 6.11. This value is consistent with the distribution model fit, the Gobas model, and molecular structure considerations. For 
pentabromophenolate, we expect $\log \mathrm{K}_{\mathrm{OW}}$ to be 2.42 . For reasons unclear, this is much higher than the experimentally determined value of 1.77 . It is interesting that the molar volume model of the previous section gives a much better prediction of Log $K_{O W}=1.81$. Extrapolation of the halophenol and halophenolate curves for PBP are represented by the dotted portions of each plot (Figure 26).

Molecular surface areas for isomers computed by MMP are identical. As in the molar volume model developed by Gobas of the previous section, the MSA-based model predicts identical partition coefficients for isomers. The solvent accessible surface area (SASA) approach used by deBruijn, et al., (1990) leads to different surface area values for difficult to isomers ${ }^{10}$ and thus different partition coefficients. At present, SASA for compounds of our interest are not available, but should lead to more accurate predictions of partition coefficients for isomers.

10 SASA is the total surface that can be in contact with a solvent obtained by a solvent-sized sphere rolled about the solute molecule. Different isomers have different inter-atomic spaces inaccessible to the solvent. 
Table 12. Molecular surface areas of halophenols, halophenolates, and selected halobenzenes.

\begin{tabular}{llll}
\hline & $\begin{array}{l}\text { MSA } \\
\left(\mathrm{nm}^{2}\right)\end{array}$ & $\log \mathrm{K}_{\text {Ow }}$ & $\log _{\mathrm{A}}$ \\
\hline Halobenzenes & & & \\
1,3,5 Trichlorobenzene & 1.560 & $4.139^{\mathrm{a}}$ & - \\
1,2,4 Trichlorobenzene & 1.560 & $4.050^{\mathrm{a}}$ & - \\
1,2,3,5 Tetrachlorobenzene & 1.745 & $4.658^{\mathrm{a}}$ & - \\
1,2,4,5 Tetrachlorobenzene & 1.745 & $4.604^{\mathrm{a}}$ & - \\
Pentachlorobenzene & 1.931 & $5.183^{\mathrm{a}}$ & - \\
Hexachlorobenzene & 2.116 & $5.731^{\mathrm{a}}$ & - \\
1,4 Dibromobenzene & 1.501 & $4.30^{\mathrm{b}}$ & - \\
1,3,5 Tribromobenzene & 1.749 & $5.26^{\mathrm{b}}$ & - \\
Hexabromobenzene & 2.494 & $6.80^{\mathrm{b}}$ & - \\
$\quad$ Halophenols & & & \\
2,4,6-Trichlorophenol & 1.687 & $3.65^{\mathrm{c}}$ & $0.15^{\mathrm{c}}$ \\
2,3,4,5-Tetrachlorophenol & 1.873 & $4.52^{\mathrm{d}}$ & $0.87^{\mathrm{d}}$ \\
2,3,4,6-Tetrachlorophenol & 1.873 & 4.28 & 0.48 \\
Pentafluorophenol & 1.505 & 2.79 & -0.85 \\
Pentachlorophenol & 2.058 & 4.77 & 1.16 \\
Pentabromophenol & 2.373 & $4.67^{*}$ & 1.77 \\
\hline
\end{tabular}
a. deBruijn (1989)
b. Gobas (1988)
c. Sieder (1995)
d. Schmidt (1995)
* Not reliable.

Table 13. Slope and intercept results from linear regression curve fitting.

\begin{tabular}{llllll}
\hline & Slope & Intercept & $\mathrm{r}^{2}$ & $\begin{array}{c}\alpha \\
\left(\frac{\mathrm{kcal}}{\mathrm{mol} \cdot \mathrm{nm}^{2}}\right)\end{array}$ & $\begin{array}{c}\Delta G_{\text {el }} \\
(\mathrm{kcal} / \mathrm{mol})\end{array}$ \\
\hline Bromobenzenes & $2.41 \pm 0.36$ & $0.83 \pm 0.71$ & 0.957 & $-3.23 \pm 0.48$ & $-2.83 \pm 0.95$ \\
Chlorobenzenes & $2.95 \pm 0.08$ & $-0.50 \pm 0.14$ & 0.997 & $-3.95 \pm 0.11$ & $-1.05 \pm 0.19$ \\
Halophenols & $3.69 \pm 0.47$ & $-2.65 \pm 0.85$ & 0.954 & $-4.95 \pm 0.36$ & $1.84 \pm 1.14$ \\
Halophenolates & $3.58 \pm 0.55$ & $-6.08 \pm 1.00$ & 0.933 & $-4.80 \pm 0.74$ & $6.43 \pm 1.34$ \\
\hline
\end{tabular}




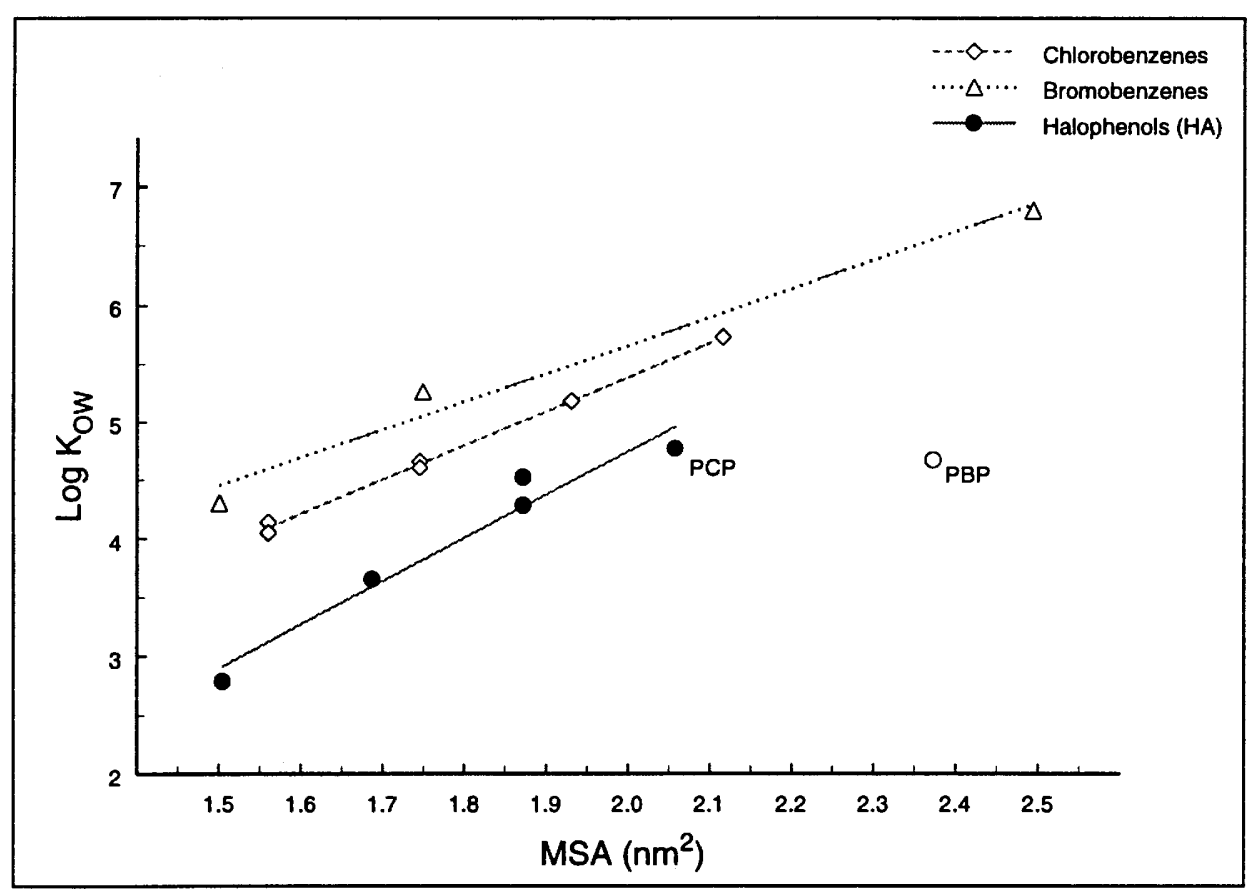

Figure 25. Plot illustrating the correlation between Log $\mathrm{K}_{\mathrm{OW}}$ and molecular surface area for halobenzenes and halophenols. 


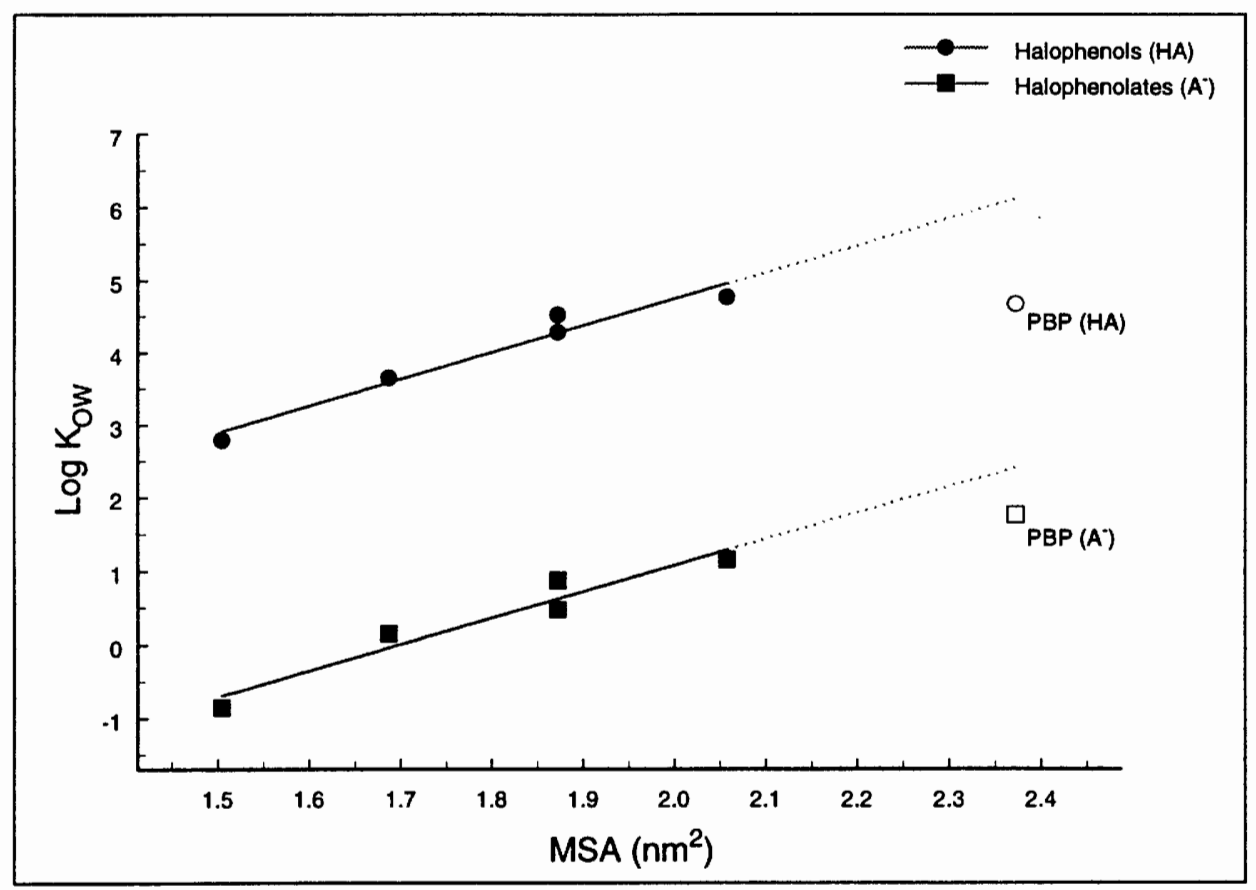

Figure 26. Plot illustrating the correlation between $\log \mathrm{K}_{\mathrm{OW}}$ and molecular surface area of halophenols and halophenolates. The dotted portions of the lines are extrapolations to predicted values for ionized and neutral PBP. 


\section{Distribution of halophenols in a lipid membrane-water system}

Escher and Scwarzenbach recently published (1996) lipid membrane-water ${ }^{11}$ partition coefficients for ionized and unionized chlorophenols including 2,3,4,6-TeCP and PCP. They reported lipid membrane-water partition coefficients $\mathrm{K}_{\mathrm{lipw}}(\mathrm{HA})$ for neutral $\mathrm{TeCP}$ and PCP to be $(2.90 \pm 0.41) \times 10^{4}$ and $(1.49 \pm 0.11) \times 10^{5}$. Ionized TeCP and PCP have partition coefficients $\mathrm{K}_{\mathrm{lipw}}\left(\mathrm{A}^{-}\right)$of $(2.44 \pm 1.69) \times 10^{3}$ and $(6.14 \pm 3.16) \times 10^{3}$, respectively.

Lipid membrane-water ${ }^{12}$ linear partition coefficients are also available for pentafluorophenolate and pentabromophenolate (Smejtek, et al. 1996). The linear partition coefficients $\beta_{x}$ for PFP (A) and PBP (A) are 0.13 and $140 \mu \mathrm{m}$. For comparison with $\mathrm{K}_{\mathrm{Ow}}$ results, we can use the relation between the linear partition coefficient and the bulk partition parameter

$$
\gamma_{x}=2 \beta_{x} / t
$$

Where the membrane thickness $t$ is $3.8 \mathrm{~nm}$ (McIntosh and Simon 1986). Lipid membrane-water partitioning parameters are given in Table 14.

The octanol-water partition coefficients $\left(g_{H A}\right.$ and $\left.K_{O W}\right)$ are similar to the lipid membrane-water results for neutral PCP and TeCP $\left(K_{o w}=6 \times 10^{4}\right.$ versus $K_{l i p w}=1.49$ x $10^{5}$ and $1.89 \times 10^{4}$ versus $\left.1.49 \times 10^{5}\right)$. However, ionized species behave much differently in the octanol-water system then their counterparts in the lipid membranewater system. The lipid membrane-water partition coefficient is about three orders of

1 L- $\alpha$-dipalmitoylphosphatidylcholine or DPPC lipid vesicles.

12 Egg-phosphatidylcholine or egg-PC lipid vesicles. 
magnitude greater than the octanol-water results for all halophenolates (see $\mathrm{K}_{\text {lipw }}\left(\mathrm{A}^{-}\right) / \mathrm{K}_{\mathrm{OW}}\left(\mathrm{A}^{-}\right)$in Table 14$)$.

Using the distribution equation (18) and the lipid parameters of PCP we can plot and compare octanol-water and lipid membrane-water partitioning over our experimental $\mathrm{pH}$ range (Figure 27). For $\mathrm{pH}$ about one or more greater than the $\mathrm{pKa}$ ( $\mathrm{pH}>5.8$ for $\mathrm{PCP}$ and $\mathrm{pH}>6.4$ for $\mathrm{TeCP}$ ) it is obvious that the correlation between $K_{O W}$ and $K_{L i p W}$ fails. We conclude that lipid membrane-water partitioning of ionized molecules can not be predicted by octanol-water results. 


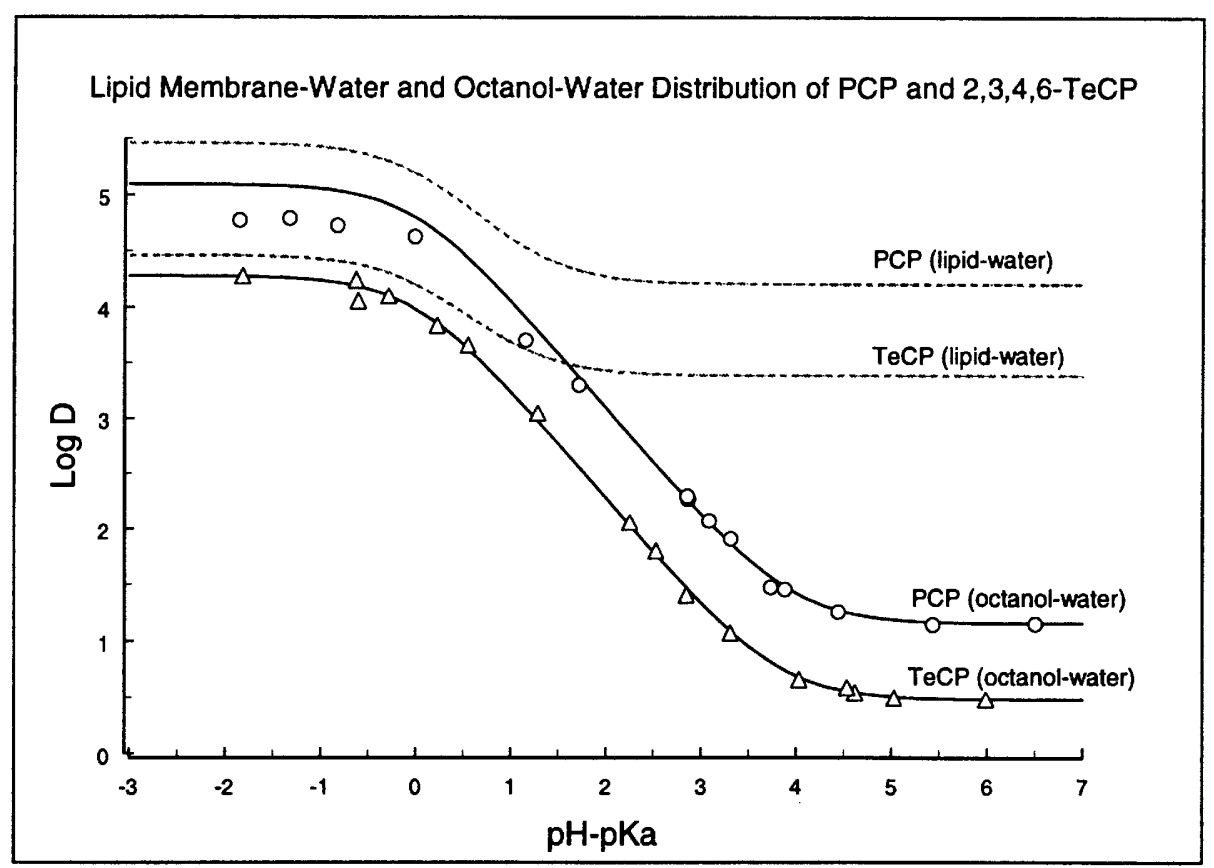

Figure 27. Comparison between the lipid membrane-water and octanol-water distribution of PCP and 2,3,4,6-TeCP.

Table 14. Octanol-water and lipid membrane-water partitioning parameters for halophenols and halophenolates.

\begin{tabular}{|c|c|c|c|c|c|}
\hline Halophenol & Kow & $\mathrm{K}_{\mathrm{lipw}}(\mathrm{HA})$ & $\mathrm{K}_{\mathrm{ow}}(\mathrm{A}-\mathrm{-}$ & $\begin{array}{c}\mathrm{K}_{\mathrm{lipw}}\left(\mathrm{A}^{-}\right) \text {or } \\
\gamma_{\mathrm{A}^{-}}\end{array}$ & $\frac{\mathrm{K}_{\mathrm{lipW}}\left(\mathrm{A}^{-}\right)}{\mathrm{K}^{-}\left(\mathrm{A}^{-}\right)}$ \\
\hline 2,3,4,6-ТеCP & $1.98 \times 10^{4}$ & $2.90 \times 10^{4 a}$ & 3.01 & $2.44 \times 10^{3 a}$ & $\frac{\mathrm{K}_{\mathrm{ow}}(\mathrm{A})}{811}$ \\
\hline PFP & 610 & - & .140 & $68^{\mathrm{b}}$ & 490 \\
\hline PCP & $6.0 \times 10^{4}$ & $1.49 \times 10^{5 \mathrm{a}}$ & 14.3 & $6.14 \times 10^{3 \mathrm{a}}$ & 429 \\
\hline PBP & $5 \times 10^{4}$ & - & 59 & $7.4 \times 10^{4 \mathrm{~b}}$ & 1300 \\
\hline
\end{tabular}

a. Escher \& Schwarzenbach, 1996.

b. Smejtek, et al., 1996. 


\section{Limits of the "shake-flask" method.}

From experimental results and correlations with molecular structure discussed in the previous sections, we conclude that $K_{O W}$ increases as function of greater molecular surface area, greater molar volume, lower $\mathrm{pKa}$, and with the number of halogen atoms in the molecule and with their higher atomic number. For PBP we expect a distribution curve similar in shape to PCP, PFP, and TeCP but shifted upwards on the $\log D$ scale. In contrast, $\log K_{O W}$ for PBP was found to be even lower than for PCP. The two respective distribution curves intersect in the low $\mathrm{pH}$ region, (Figure 28). The distribution ratio for PCP also deviates, to a lesser extent, from the model predictions and from the literature value. ${ }^{13}$ Measurement errors cannot explain the deviation of the distribution of PCP and PBP from the model.

Many authors (deBruijn, et al. 1989; Brooke, et al. 1986; USEPA 1980) report that the shake flask method produces erroneous results for highly hydrophobic chemicals ( $\left.\log K_{O W}>5\right)$. The most likely explanation is that octanol emulsions resist centrifugation and remain in the aqueous phase. For low aqueous phase concentrations $([\mathrm{HA}+\mathrm{A}]<1 \mu \mathrm{M})$ micelles of high, localized phenol concentrations become the major contributors to UV absorbance. Since the distribution curves of PBP and PCP intersect at about $\log D=4.8$, this is probably the upper limit of our "shake flask" method (Figure 28).

\footnotetext{
${ }^{13}$ The partition coefficient of PBP is not available from the literature, but $\log K_{\text {ow }}$ of PCP is about 5.1, (Jafvert, et al. 1990). 
Some authors propose alternate methods of measuring $\mathrm{K}_{\mathrm{Ow}}$ that minimize octanol emulsions. Reverse phase-high performance liquid chromatography (RPHPLC) and a generator column method are discussed in the literature (deBruijn, et al. 1989), but they either have calibration difficulties or are time-consuming. A relatively simple approach used by Brooke, et al. (1986) and deBruijn, et al. (1989) is the "slow stirring" method. This method minimizes formation of octanol emulsions in water and may be useful in further partitioning experiments involving very hydrophobic compounds. In this method, the octanol mixture is carefully added to about 1 liter of buffer in an Erlenmeyer flask. By careful stirring and temperature control, octanol emulsions are minimized and high $\log K_{O W}$ values up to 8 were measured. An attractive feature of the method is that samples from the buffer phase are extracted without pipette contact with the octanol layer. Nanomolar aqueous concentrations below the limit of UV-Vis spectrophotometric resolutions are measured by HPLC or gas chromatography (GC).

It is proposed that the "slow-stirring" method be used in our laboratory in further studies of the distribution of highly hydrophobic compounds between octanol and water. 


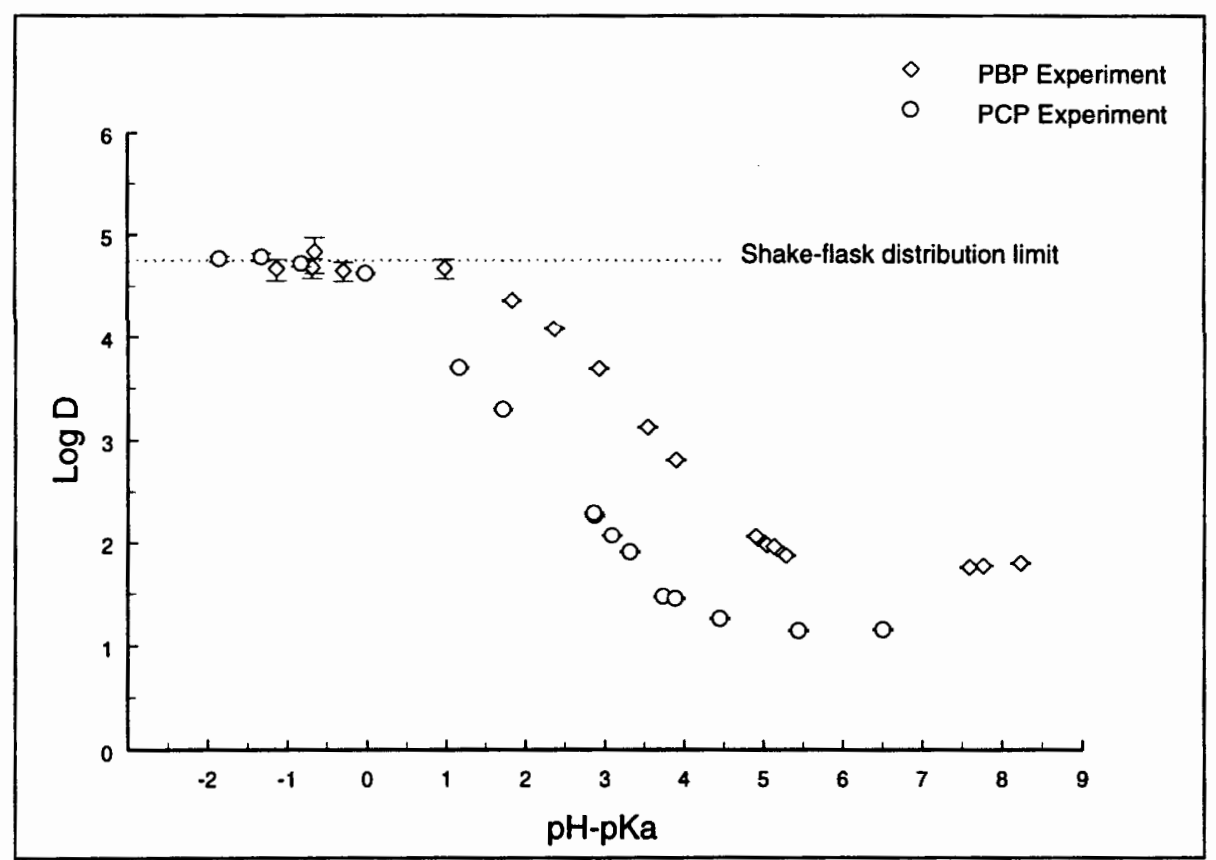

Figure 28. PCP and PBP data merge in the low $\mathrm{pH}$ region. $\log \mathrm{K}_{\mathrm{OW}}$ of 4.8 is probably the upper limit of the "shake flask" method. 


\section{Conclusions}

We can make a number of conclusions from the results of this study:

1) The logarithmic octanol-water partition coefficients ( $\left.\log K_{O W}\right)$ for the neutral species of 2,3,4,6-tetrachlorophenol, pentafluorophenol, pentachlorophenol, and pentabromophenol are: 4.28 (TeCP), 2.79 (PFP), 4.77 (PCP), and approximately 4.67 (PBP). $\log K_{O w}$ for the ionized species of each phenol is: 0.48 (TeCP), -0.85 (PFP), 1.16 (PCP), and 1.77 (PBP). We have observed relationships between the distribution parameters and the molecular structure of these phenols.

2) Octanol-water partitioning behavior is dependent on the physical and electrostatic properties of the solute. Molecular surface area, molar volume, quantity and atomic number of the halogens of the phenyl ring, and electric charge are all factors determining the octanol-water distribution of halogen substituted phenols.

3) The octanol-water model is a poor predictor for the partitioning of ionized halogen substituted phenols between the lipid membranes of organisms and aqueous media.

4) If polluted soils and sediments are sources of halogenated phenols in aquatic systems, the octanol-water distribution of the ionized species suggest that the aqueous concentration of halogenated phenols will be greater than expected from the distribution of the unionized species. 


\section{Appendix}

\section{Evaluation of molecular modeling software}

We tested two commercial molecular modeling packages for use in this study. Molecular Modeling Pro (MMP) and its companion Molecular Analysis Pro were obtained from Windowchem Software. A demo of ACD LogP and ACD Chemsketch were obtained form Advanced Chemical Development Inc. These two packages contain functions for evaluating Log $K_{\text {Ow. }}$ A third program tested, CLogP (BioByte Corp.), is specifically designed for estimating octanol-water partition coefficients.

The three programs for predicting partition coefficients use estimation techniques created from large data bases of $K_{O W}$ values. Fractional contributions from atom type, bond type, and other compound properties are used to estimate the partition coefficient. Results obtained by these molecular modeling software packages are compared with our experimental results in Table A1. ACD and CLogP also predict different values for isomers, consistent with the literature and experiment.

ClogP gives partition coefficients with the poorest correlation to experimental and literature values. This poor correlation between predicted and experimental $\mathrm{K}_{\mathrm{OW}}$ for some types of compounds was also reported by Schüürmann and Klein (1988). CLogP is also more difficult to use than the other mouse driven programs since the clumsy Smiles notation is used to specify the molecular structure of compounds, (for example, PFP in Smiles notation is $c 1(F)=c(O) c(F)=c(F) c(F)=c 1(F))$.

Overall, ACD predicts $\log K_{\text {OW }}$ values in closest agreement with our experiment. ACD Chemsketch has a more attractive interface, a good log $K_{O W}$ 
prediction function, and offers more shortcut tools than MMP and MAP. However, the package of MMP and MAP supplemented with RASMOL molecular imaging software (created R. Sayle and available in the public domain) offers most of the features of the ACD package, but at a more attractive price $(\$ 149.95$ compared to $\$ 499$ at educational prices). 
Table A1. Computed and experimental $\log K_{O W}($ or $\log P$ ) values of halogenated phenols.

\begin{tabular}{|c|c|c|c|c|c|}
\hline Compound & $\begin{array}{l}\text { ACD } \\
\text { LogP }\end{array}$ & CLogP & $\begin{array}{l}\text { MMP } \\
\text { LogP }\end{array}$ & Literature & $\begin{array}{c}\text { Experiment } \\
\log \mathrm{g}_{\mathrm{HA}}=\log \mathrm{P}\end{array}$ \\
\hline 2,4,6-Trifluorophenol ${ }^{\mathrm{a}}$ & 2.24 & 1.917 & 1.904 & Not Available & NA \\
\hline 2,4,6-Trichlorophenol ${ }^{\mathrm{a}}$ & 3.58 & 3.367 & 3.614 & $3.72^{b}, 3.75^{c}, 3.69^{d}, 3.38^{e}$ & $3.65^{\mathrm{h}}$ \\
\hline 2,4,6-Tribromophenol ${ }^{\mathrm{a}}$ & 4.33 & 3.917 & 4.604 & $3.96,4.13,4.23^{f}$ & $\mathrm{NA}$ \\
\hline 2,4,6-Triiodophenol ${ }^{\mathrm{a}}$ & 3.88 & 4.557 & 4.844 & NA & NA \\
\hline $2,3,4,5$-Tetrachlorophenol ${ }^{\mathrm{a}}$ & 4.39 & 4.058 & 4.327 & $4.87^{b}, 4.68^{d}, 4.21^{f}$ & $4.52^{\mathrm{i}}$ \\
\hline 2,3,4,6-Tetrachlorophenol & 4.17 & 3.848 & 4.327 & $4.42^{b}, 4.42^{c}, 4.34^{d}, 4.45^{f}$ & 4.28 \\
\hline Pentafluorophenol & 2.66 & 2.213 & 2.19 & $3.23^{9}$ & 2.79 \\
\hline Pentachlorophenol & 4.78 & 4.323 & 5.04 & $\begin{array}{c}5.24^{\mathrm{b}}, 5.04^{\mathrm{c}}, 5.08^{\mathrm{d}}, 5.01^{\mathrm{e}}, \\
5.12^{\mathrm{f}}, 5.09^{\mathrm{g}}\end{array}$ & 4.77 \\
\hline Pentabromophenol & 6.10 & 4.853 & 5.79 & NA & 4.67 \\
\hline
\end{tabular}
a. Included for evaluation purposes only.
b. Schellenberg, et al. 1984
c. Saito, et al. 1991
d. Xie, et al. 1984
e. Westall, et al. 1985
f. Hansch, et al. 1995
g. Jafvert, et al. 1990
h. Sieder 1995
i. Schmidt 1995 


\section{References}

W. Arnold, U. Zimmermann, W. Pauli, M. Benzing, C. Niehrs, and J. Ahlers, "The comparative influence of substituted phenols (especially chlorophenols) on yeast cells assayed by electro-rotation and other methods.," Biochim. Biophys. Acta 942, 83-95 (1988).

Phillip R. Bevington, Data Reduction and Error Analysis for the Physical Sciences (McGraw-Hill, Case Western Reserve University, 1969).

B. T. Bowman and W. W. Sans, "Determination of Octanol-Water Partitioning Coefficients $\left(\mathrm{K}_{\mathrm{OW}}\right)$ of 61 Organophosphorus and Carbonate Insecticides and Their Relationship to Respective Water solubility (S) Values," Journal of Environmental Science and Health B18(6), 667-683 (1983).

D. N. Brooke, A. J. Dobbs, and N. Williams, "Octanol:Water Partition coefficients (P): Measurement, Estimation, and Interpretation, Particularly for Chemical with $\mathrm{P}>10^{5}, "$ Ecotoxicology and Environmental Safety 11, 251-260 (1986).

CRC Handbook of Chemistry and Physics, 51 (The Chemical Rubber Co., 1970).

J. de Bruijn, F Busser, W. Seinen, and J. Hermens, "Determination of Octanol/Water Partition Coefficients for Hydrophobic Organic Chemicals with the "SlowStirring" Method," Environmental Toxicology and Chemistry 8, 499-512 (1989).

B. I. Escher and R. P. Schwarzenbach, "Partitioning of substituted phenols in liposomewater, biomembrane-water, and octanol-water systems," Environ. Sci. Technol 30, 260-270 (1996).

P. C. Gobas, J. M. Lahittete, G. Garofalo, W. Y. Shiu, and D. Mackay, "A Novel Method for Measuring Membrane-Water Partition Coefficients of Hydrophobic Organic Chemicals: Comparison with 1-Octanol-Water Partitioning," Journal of Pharmaceutical Sciences 77 (3), 265-272 (March, 1988).

C. Hansch, A. Leo, and D. Hoekman, Exploring QSAR Hydrophobic, Electronic, and Steric Constants (American Chemical Society, Washington DC, 1995).

H. A. Hattemer-Frey and C. C. Travis, "Pentachlorophenol: Environmental Partitioning and Human Exposure," Archives of Environmental contamination and Toxicology 18, 482-489 (1989).

P. V. Hodson, D. G. Dixon, and K. L. Kaiser, E., "Estimating the Acute Toxicity of Waterborne Chemicals in Trout From Measurements of Median Lethal Dose and Octanol-Water Partition Coefficient," Environmental Toxicology and Chemistry 7, 443-454 (1988). 
IUPAC, Ionisation Constants of Organic Acids in Aqueous Solution, Chemical Data Series, vol. 23 (Pergamon Press, 1979).

C. T. Jafvert, J. C. Westall, E. Grleder, and R. P. Schwarzenbach, "Distribution of Hydrophobic Ionogenic Organic Compounds Between Octanol and Water: Organic Acids," Environ. Sci. Technol 24 (12), 1795-1803 (1990).

C. Kittel and H. Kroemer, Thermal Physics, 2nd edition (W.H. Freeman and Company, New York, 1980), pp. 262-274.

Albert L. Lehninger, Principles of Biochemistry (Worth Publishing, Inc., 1982).

T. J. McIntosh and S. A. Simon, "Hydration Force and Bilayer Deformation: A Reevaluation," Biochemistry 25, 4058-4066 (1986).

H. Mussalo-Rauhamaa, H. Pyysalo, and K. Antervo, "The Presence of Chlorophenols and Their Conjugates in Finnish Human Adipose and Liver Tissues," The Science of the Total Environment 83, 161-172 (1989).

H.-H. Perkampus, UV-VIS Spectroscopy and Its Applications, translated by H. C. Grinter and T. L. Threlfall (Springer Laboratory, 1992), pp. 1-25.

H. Saito, M. Sudo, T. Shigeoka, and F. Yamauchi, "In vitro cytotoxicity of chlorophenols to goldfish GF-Scale (GFS) cells and quantitative structure-activity relationships," Environmental Toxicology and Chemistry 10, 235-241 (1991).

G. Schüürmann and W. Klein, "Advances in Bioconcentration Prediction," Chemosphere 17 (8), 1551-1574 (1988).

K. Schellenberg, C. Leuenberger, and R. P. Schwarzenbach, "Sorption of Chlorinated Phenols by Natural Sediments and Aquifer Materials," Environmental Science and Technology 18 (9), 652-657 (1984).

Piet O. Schmidt, "Origins of Effective Charge of Multivalent Ions at a Membrane/Water Interface and Distribution of 2,3,4,5-Tetrachlorophenol in a Membrane Model System.," Master's thesis (Portland State University, Portland, OR, 1995).

R. P. Schwarzenbach and J. C. Westall, "Sorption of hydrophobic trace organic compounds in groundwater systems," Wat. Sci. Tech. 17, 39-55 (1985).

Isolde Sieder, "Electrostatic Interactions at Water-Membrane Interfaces and Distribution of 2,4,6-Trichlorophenol in a Membrane Model System," Master's thesis (Portland State University, Portland, OR, 1995). 
P. Smejtek, A. Blochel, and S. Wang, "Hydrophobicity and Sorption of Chlorophenolates to Lipid Membranes," Chemosphere, in press., 1996).

P. Smejtek and S. Wang, "Distribution of Hydrophobic Ionizable Xenobiotics between Water and Lipid Membranes: Pentachlorophenol and Pentachlorophenate. A Comparison with Octanol-Water Partition," Archives of Environmental contamination and Toxicology 25, 394-404 (1993).

USEPA., Octanol/Water Partition Coefficient. EPA-560/11-80-027, p. 79-119, Test Data Development Standards: Physical, Chemical, and Persistence Characteristics (U.S. Environmental Protection Agency., 1980).

L. Wang, R. Govind, and R. A. Dobbs, "Sorption of Toxic Organic Compounds on Wastewater Solids: Mechanism and Modeling," Environmental Science and Technology 27 (1), 152-158 (1993).

J. C. Westall, C. Leuenberger, and R. P. Schwarzenbach, "Influence of $\mathrm{pH}$ and Ionic Strength on the Aqueous-Nonaqueous Distribution of Chlorinated Phenols," Environmental Science and Technology 19 (2), 193-198 (1985).

S. R. Wild and K. C. Jones, "Pentachlorophenol Exposure in the UK Environment II: Human Exposure and an Assessment of Pathways," Chemosphere 24 (7), 847-855 (1992).

T. M. Xie, B. Hulthe, and S. Folestad, "Determination of Partition Coefficients of Chlorinated Phenols, Guaiacols and Catechols by Shake-Flask GC and HPLC," Chemosphere 13 (3), 445-459 (1984).

Geoffrey Zubay, Biochemistry, 2nd edition (Macmillan Publishing Company, 1983), pp. 176-209. 